\title{
CRITICAL ROLE OF ELECTRON LEAK FROM MITOCHONDRIAL ELECTRON TRANSPORT CHAIN COMPLEX I IN OXYGEN SENSING IN THE RABBIT DUCTUS ARTERIOSUS
}

by

Austin Read

A thesis submitted to the Department of Medicine

In conformity with the requirements for

the degree of Master of Science in Translational Medicine

\author{
Queen's University \\ Kingston, Ontario, Canada
}

(December, 2021)

Copyright (C) Austin D. Read, 2021 


\begin{abstract}
Background: The ductus arteriosus (DA) connects the pulmonary trunk to the descending thoracic aorta, allowing placentally oxygenated blood to bypass the non-ventilated fetal lungs and enter the systemic circulation. The fetus' systemic arterial oxygen tension (pO2) is $<40$ $\mathrm{mmHg}$, maintaining the DA in a state of hypoxic vasodilation. As pO2 rises with the first breath, the DA constricts and becomes functionally closed within minutes. The oxygen sensor responsible for initiating this vasoconstrictor effect resides within the mitochondria of DA smooth muscle cells (DASMC). At birth, increased pO2 leads to increased reactive oxygen species (ROS) production by complexes I and III of the electron transport chain (ETC). These ROS act as diffusible signaling molecules and inhibit redox sensitive potassium channels causing DASMC depolarization and vasoconstriction. Complex I and III inhibitors, rotenone and antimycin A, relax the DA ex vivo by decreasing normoxic ROS production by the mitochondria, but also alter mitochondrial respiration. A new generation of electron leak suppressors, S1QEL and S3QEL, which decrease electron leak from complexes I and III respectively, can selectively decrease ROS production without inhibiting the metabolic function of the ETC complexes. These new leak suppressors may offer better understanding of the physiology of $\mathrm{O}_{2}$ sensing in the DA and also offer new therapeutic avenues for controlling DA tone in vivo.

Methods/Results: DA tissues were obtained from term rabbit kits. Using the ring bath model for measuring vessel tone ex vivo, we have shown that S1QEL causes a vasodilatory effect in oxygen induced pre-constricted DAs. In rabbit DASMC, S1QEL decreases oxygen consumption rates (OCR), while maintaining key parameters related to mitochondrial respiration. In DASMC isolated from humans, S1QEL blunts oxygen induced increases in superoxide generation. In vivo, S1QEL reverses oxygen induced constriction of the DA in term rabbit kits.
\end{abstract}


Conclusions: S1QEL, but not S3QEL, causes reversal of DA constriction both ex vivo and in vivo, suggesting an importance for Complex I electron leak during DA oxygen sensing. S1QEL decreases superoxide production in DASMC exposed to normoxia, but maintains key parameters of mitochondrial respiration, confirming the importance of ROS produced by Complex I in DA oxygen signaling. 


\section{Acknowledgments}

I would like to start by thanking my supervisor and mentor, Dr. Stephen Archer for his guidance, patience and enthusiasm throughout my project. Dr. Archer's support has extended beyond the scope of my master's study and he has continued to support me as I prepare to graduate from the Translational Medicine program. I would also like to thank Dr. Kimberly Dunham-Snary, who has been another invaluable mentor to me, both inside and outside the lab. Dr. Dunham-Snary's knowledge of vascular oxygen sensing and her approach to the scientific method has helped me approach challenging research questions and grow as a scientist. I would also like to acknowledge the efforts of my thesis advisory committee members, Dr. Mark Ormiston and Dr. Bernard Thébaud, for taking the time to meet with me, providing insightful comments and critical questions which have helped shaped the direction of my research. Finally, I would like to thank the Archer lab and QCPU staff, in particular Rachel Bentley, Ashley Martin, Elahe Alizadeh, and Patricia Lima, who have been essential collaborators in my research efforts and without whom my research project would not have been possible. 


\section{Co-Authorship}

Rachel A Bentley ${ }^{1}$, Ashley Y Martin ${ }^{1}$, Jeffrey D Mewburn ${ }^{1}$, Elahe Alizadeh ${ }^{2}$, Danchen $\mathrm{Wu}^{1}$,

Patricia AD Lima ${ }^{2}$, Kimberly J Dunham-Snary ${ }^{1,3}$, Bernard Thébaud ${ }^{4}$, Willard Sharp ${ }^{5}$, Stephen L $\operatorname{Archer}^{1,2}$

1 - Department of Medicine, Queen's University, Kingston, ON, CAN

2 - Queen's CardioPulmonary Unit, Queen's University, Kingston, ON, CAN

3 - Department of Biomedical and Molecular Sciences, Queen's University, Kingston, ON, CAN

4 - Department of Pediatrics, University of Ottawa, Ottawa, ON, CAN

5 - Department of Medicine, The University of Chicago, Chicago, IL, USA 


\section{Table of Contents}

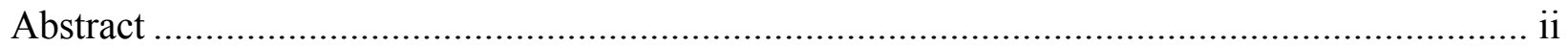

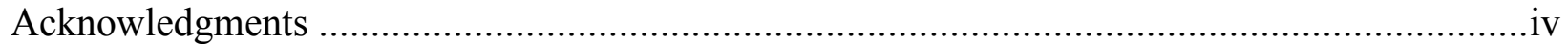

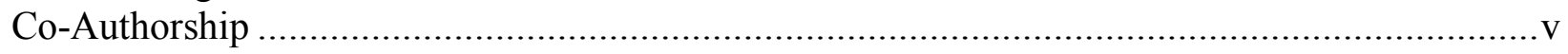

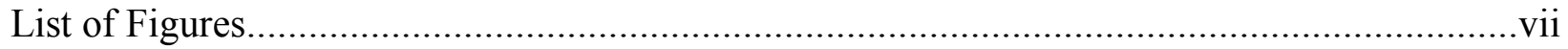

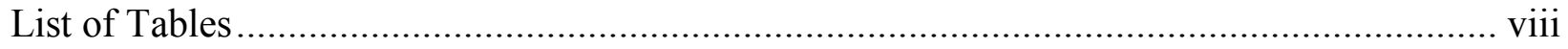

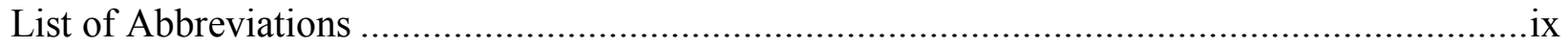

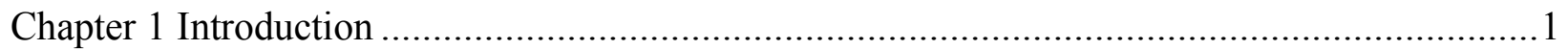

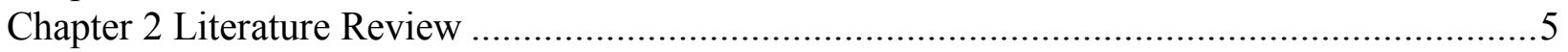

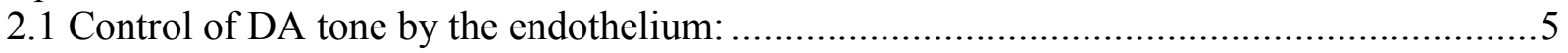

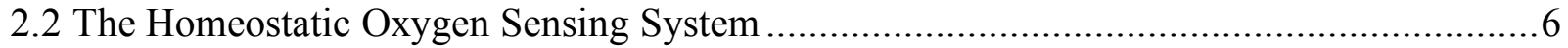

2.3 Ion channels as effectors of oxygen signaling ............................................................

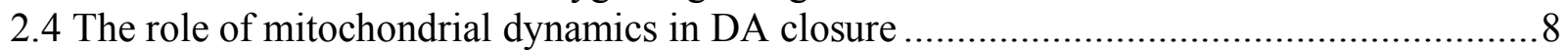

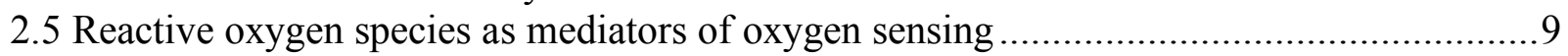

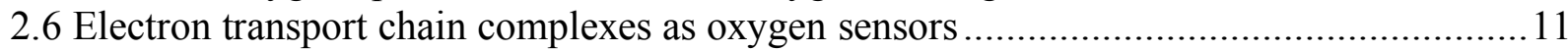

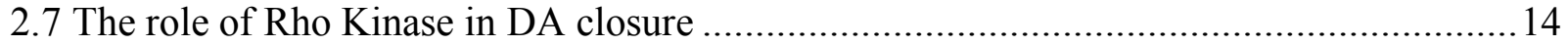

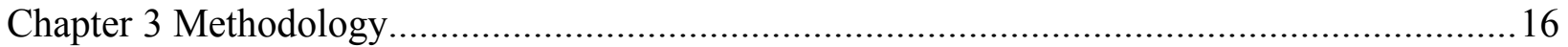

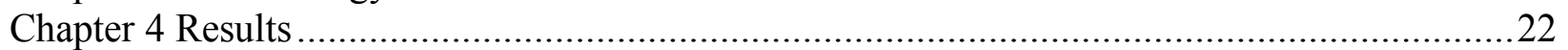

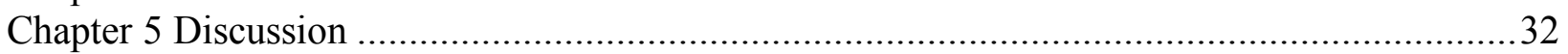

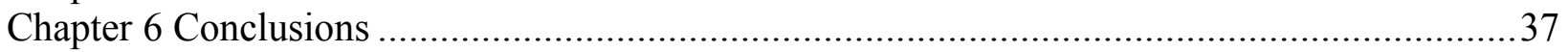

Chapter 7 Mitochondrial Iron-Sulfur Clusters: Structure, Function, and an Emerging Role in

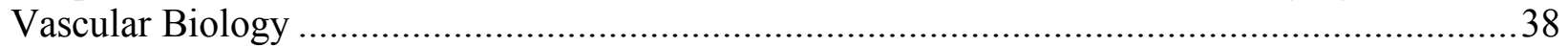

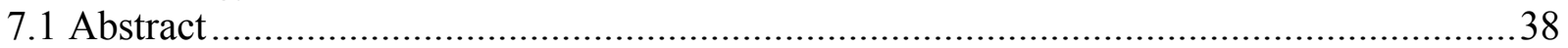

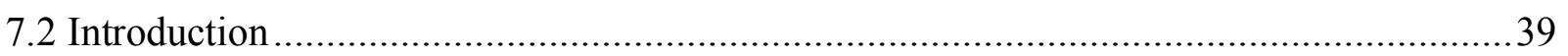

7.3 Mitochondria and endosymbiosis..............................................................................

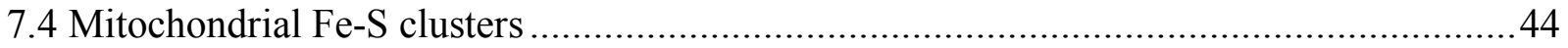

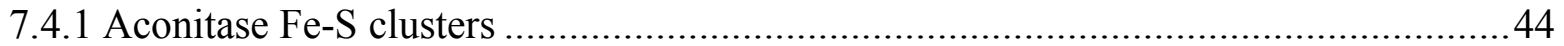

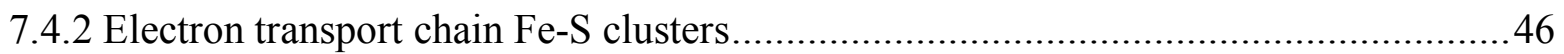

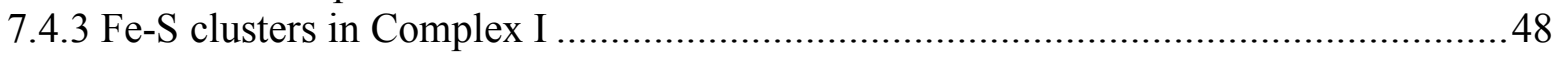

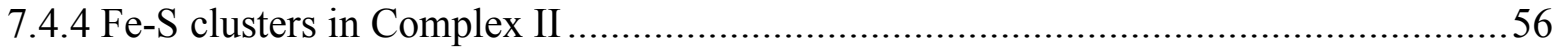

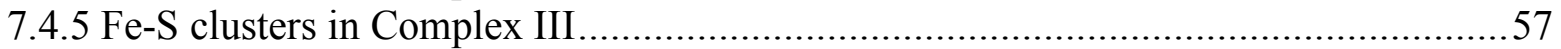

7.5 Fe-S clusters and the Homeostatic Oxygen-Sensing System...........................................6 60

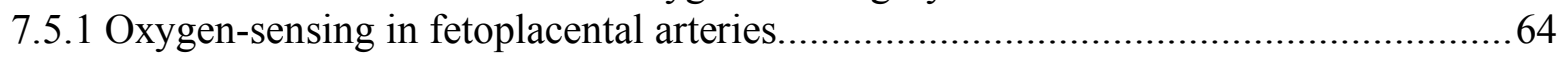

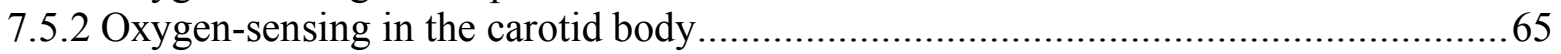

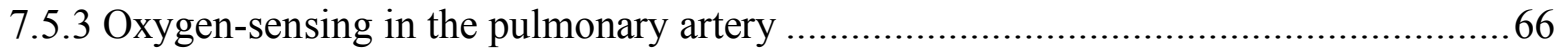

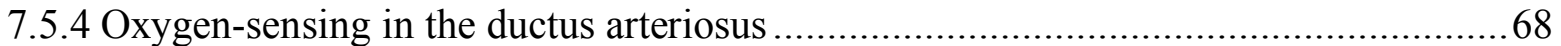

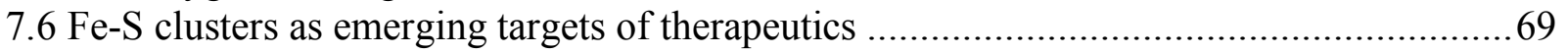

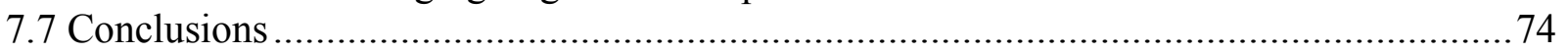

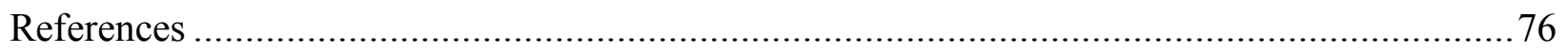




\section{List of Figures}

Figure 1 Cardiovascular adaptations of the fetal heart .......................................................2

Figure 2 Effects of electron leak suppressors and ETC complex inhibitors on DA vessel tone ...23

Figure 3 Isolation of DASMC using flow cytometry ..........................................................24

Figure 4 Impact of electron leak suppressors and rotenone on respiratory parameters .............26

Figure 5 Impact of electron leak suppressors and rotenone on mitochondrial respiration .........27

Figure 6 Superoxide measurement in hDASMC using MitoROS 580 ....................................29

Figure 7 Effects of electron leak suppressors and rotenone on superoxide production...............30

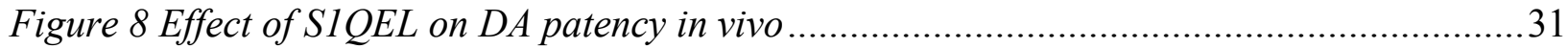

Figure 9 The most common forms of $\mathrm{Fe}-\mathrm{S}$ clusters found in biological systems ........................40

Figure 10 Overview of endosymbiont hypothesis ............................................................ 42

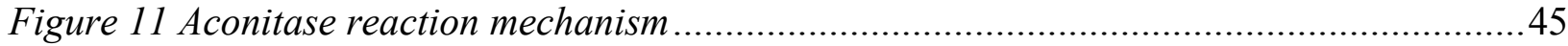

Figure 12 Simplified version of the mitochondrial ETC .........................................................48

Figure $13 \mathrm{Fe}$-S chain within Complex I......................................................................... 51

Figure 14 Q-cycle within Complex III ..........................................................................59

Figure 15 The specialized tissues of the Homoeostatic Oxygen Sensing System (HOSS) ............62

Figure 16 PASMC mitochondrion during normoxia and hypoxia ..........................................68 


\section{List of Tables}

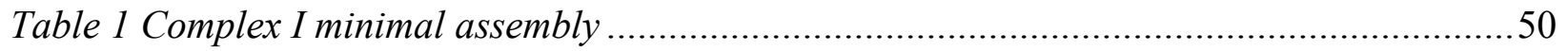

Table 2 Summary of drugs targeting Fe-S clusters ......................................................... 71 


\section{List of Abbreviations}

1- DA: Ductus Arteriosus

2- PDA: Patent Ductus Arteriosus

3- PPHN: Persistent Pulmonary Hypertension of the Newborn

4- HPV: Hypoxic Pulmonary Vasoconstriction

5- PA: Pulmonary Artery

6- SMC: Smooth Muscle Cell

7- DASMC: Ductus Arteriosus Smooth Muscle Cell

8- PASMC: Pulmonary Artery Smooth Muscle Cell

9- hDASMC: Human Ductus Arteriosus Smooth Muscle Cell

10-rbDASMC: Rabbit Ductus Arteriosus Smooth Muscle Cell

11-ETC: Electron Transport Chain

12- IMM: Inner Mitochondrial Membrane

13- IMS: Inter Membrane Space

14- OCR: Oxygen Consumption Rate

15-ROS: Reactive Oxygen Species

16- S1QEL: Suppressor of Site $I_{Q}$ Electron Leak

17- S3QEL: Suppressor of Site $\mathrm{III}_{\mathrm{Q}}$ Electron Leak

18-HOSS: Homeostatic Oxygen Sensing System

19- MLC: Myosin Light Chain

20- LV: Left Ventricle

21-RV: Right Ventricle

22- MIP: Maximum Intensity Projection 
23-ROCK-1: Rho-associated coiled-coil kinase 1 


\section{Chapter 1}

\section{Introduction}

With the first breath, the newborn's circulatory system must quickly adapt to obtaining oxygen through respiration, rather than through their mother's circulation. As a fetus, the heart is designed to allow oxygenated blood coming from the placenta to bypass the developing lungs, and be re-directed to the systemic circulation. This is accomplished by the constriction of the PA in utero, as well as the presence of the DA, a specialized blood vessel connecting the pulmonary trunk to the descending aorta. When patent in utero, the DA acts to divert blood away from the unventilated pulmonary circulation (Figure 1A).

In the womb, the fetus' $\mathrm{pO} 2$ is $<40 \mathrm{mmHg}$, maintaining the $\mathrm{DA}$ in a state of hypoxic vasodilation while simultaneously eliciting PA vasoconstriction [1]. At birth, a rise in oxygen in the environment causes the DA to close, and conversely the PA to vasodilate, allowing blood to travel through the newly ventilated lungs and eliminating the fetal right to left shunting of blood (Figure 1B). Closure of the DA after birth reflects initial functional closure, mediated by the DA constriction, reflecting contraction of smooth muscle cell (SMC) within the DA's media, followed by subsequent anatomical closure resulting in the creation of a fibrous ligamentum arteriosum [2]. Functional closure of the ductus typically occurs within the first 48 hours of life in full-term babies [3]. Anatomical closure of the ductus is a gradual process which follows functional closure, and involves proliferation of the intima and media, creating mucoid filled spaces and causing fragmentation of the internal elastic lamina [4]. 
A) Fetal Circulation

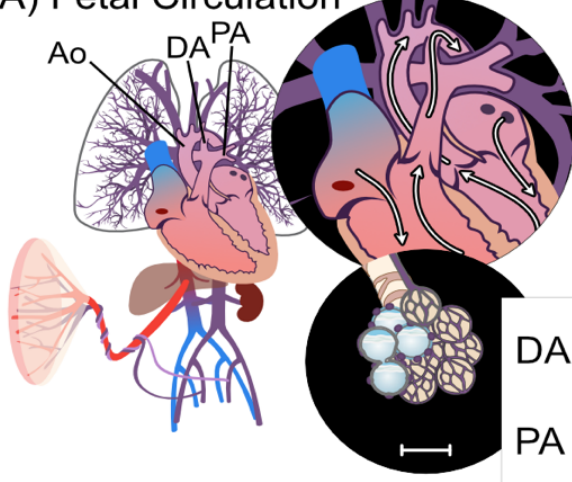

C) PPHN

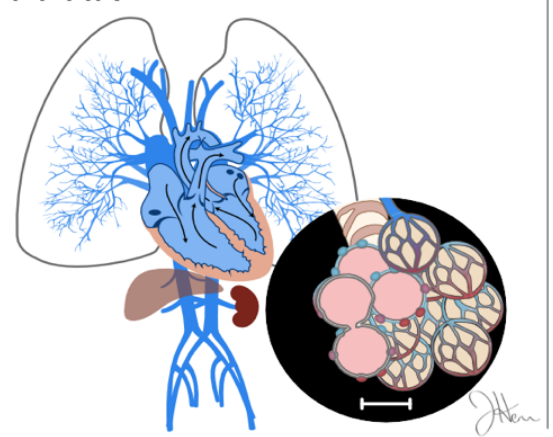

B) Newborn

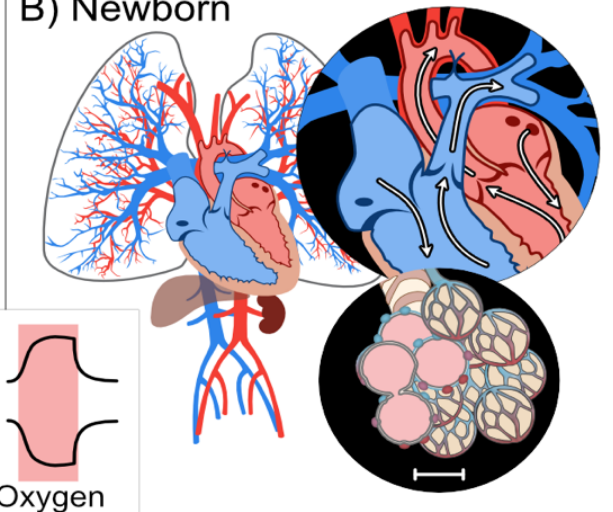

D) PDA

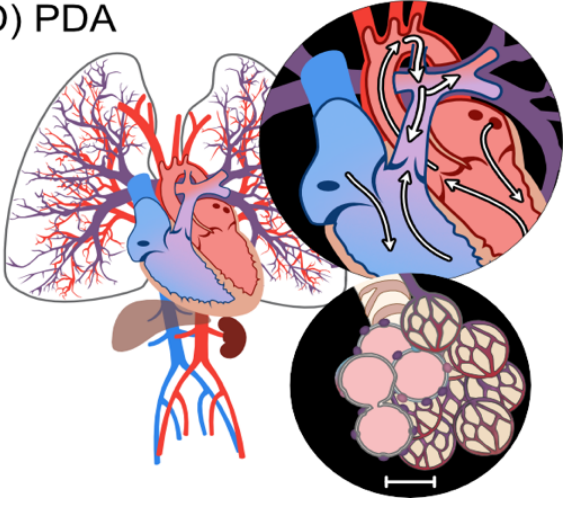

Figure 1 Cardiovascular adaptations of the fetal heart. In panel (A) the fetal circulation is shown, with the majority of blood exiting the right ventricle bypassing the pulmonary arteries $(P A)$ to instead travel to the descending aorta (Ao) through the ductus arteriosus $(D A)$. In panel $(\boldsymbol{B})$, with the first breath, oxygenation causes opposing changes in vessel tone in the PA and DA; the DA constricts and the PA dilates. Together these changes establish the neonatal circulation and ensure perfusion of the newly ventilated lung. In panels $(\boldsymbol{C})$ and $(\boldsymbol{D})$, two forms of congenital heart disease in preterm infants are shown. In panel $(\boldsymbol{C})$, persistent pulmonary hypertension of the newborn (PPHN) impairs oxygenation as a result of ongoing constriction of fetal PAs and increased pulmonary vascular resistance. In panel (D), patent ductus arteriosus (PDA) allows shunting of deoxygenated blood to the aorta resulting in cyanosis and heart failure.

Failure of the DA to close (patent DA [PDA], Figure 1D) accounts for approximately 5$10 \%$ of all congenital heart disease, and is associated with heart failure, necrotizing enterocolitis, intraventricular hemorrhage, renal dysfunction and chronic lung disease [5,6]. Although the incidence of PDA is fairly low for full-term infants (57 per 100,000), a third of preterm infants with a birthweight between 501-1500 g have PDA, with 55\% of infants who weigh $<1000 \mathrm{~g}$ (extremely low birth weight) being affected [7]. Additionally, $60-70 \%$ of preterm infants $<28$ weeks' gestation require medical or surgical treatment in order to correct their PDA. 
The control of vascular tone within the DA, although reinforced by vasoactive compounds produced by the endothelium, is intrinsic to the smooth muscle cells within the DA media. Our prior research has shown that during normoxia/hypoxia, these cells alter their production of reactive oxygen species (ROS), changing the redox state of the cell and causing downstream activation/inactivation of redox-sensitive proteins [8, 9]. Complexes I and III within the mitochondrial electron transport chain (ETC) are believed to be the sensors of arterial pO2, responding to changes in oxygen content by altering the production of ROS (notably hydrogen peroxide). These redox mediators act as oxidant signaling molecules, diffusing to the cell membrane where they oxidize and inhibit voltage-gated potassium channels (Kv). These $\mathrm{Kv}$ channels when inhibited depolarize the DASMC, activating the voltage-gated, large conductance calcium channel $\left(\mathrm{Ca}_{\mathrm{L}}\right)$, causing calcium influx and SMC contraction [10].

This thesis seeks to explore the mechanisms by which the DA is able to sense changes in blood pO2 and initiate vessel constriction and anatomical closure. Specifically, we examine the role of electron leak suppressors, S1QEL and S3QEL, small molecules which are capable of selectively decreasing electron leak and ROS production by ETC complexes I and III, respectively. Within the past decade, high throughput screening has allowed for the identification of several site-specific suppressors of electron leakage in the inner mitochondrial membrane. S1QELs (Suppressors of site $\mathrm{I}_{\mathrm{Q}}$ electron leak) and S3QELs (Suppressors of site $\mathrm{III}_{\mathrm{Q}}$ electron leak) are small molecules which prevent superoxide production at complexes I and III of the ETC, respectively $[11,12]$. Their specificity and potency may prove valuable in determining the relative importance of different leakage sites within the ETC to ROS production and DA constriction in response to the rise in oxygen at birth. In addition to further insights these compounds may provide on the relative importance of ETC complex I vs III to oxygen signaling, 
they may also reveal the relative importance of changes in ETC-derived ROS production versus changes in mitochondrial metabolism which accompanies rising pO2 during DA oxygen sensing. S1QEL and S3QEL selectively block electron leak from complex I and III respectively, by inhibiting formation of superoxide anions from a single $\mathrm{Q}$ site in each megacomplex (site $\mathrm{I}_{\mathrm{Q}}$ and $\mathrm{III}_{\mathrm{Q}}$, respectively), without blocking forward electron flow or substrate utilization. Thus, electron leak suppressors allow us to study ROS signalling without disturbing mitochondria membrane potential $(\Delta \Psi \mathrm{m})$ or oxidative phosphorylation. We hypothesize that these electron leak suppressors will modulate DA tone via alterations in ROS production, without effecting mitochondrial metabolism. Modulation of ROS production by the actions of S1QEL and S3QEL may provide novel therapeutic avenues for controlling DA tone in newborns with congenital heart abnormalities. 


\section{Chapter 2}

\section{Literature Review}

\subsection{Control of DA tone by the endothelium:}

Several endothelial derived mechanisms contribute to the vasodilation of the DA in utero. These mechanisms include pO2-dependent production of endothelial derived substances which act to cause relaxation of the DA, including prostaglandin $\mathrm{E} 2\left(\mathrm{PGE}_{2}\right)$, or which induce constriction, such as endothelin-1 (ET-1) $[13,14]$. The expression of these compounds has been shown to be developmentally regulated, indicating a possible reason for the increased incidences of PDA observed in preterm infants compared to full-term.

Studies by Coceani et al. have shown that ET-1 acts as a vasoconstrictor involved in DA closure [13]. ET-1 is 21 amino acid peptide synthesized within the wall of the DA, and is thought to promote closure through a cytochrome P-450 dependent monooxygenase reaction $[15,16]$. Although endothelin-1 has been shown to be a potent vasoconstrictor in vitro, our lab previously showed that effective blockade of the endogenous ET-1 pathway has no effect on O2-induced constriction of the term human DA, suggesting that although ET-1 may reinforce the actions of oxygen on DA tone, it is not necessary for closure [17].

Prenatal patency of the ductus is maintained in part by vasodilating prostaglandins, produced by the cyclooxygenase pathway [18]. Of these prostaglandins, $\mathrm{PGE}_{2}$ in particular has been shown to be a potent ductus relaxant in vitro and in vivo $[14,19]$. $\mathrm{PGE}_{2}$ synthesis has also been shown to be greater in premature than full-term infants, providing a possible rationale for the greater patency observed in the preterm DA [20]. Inhibition of prostaglandin synthesis, as occurs with cyclooxygenase inhibitors (i.e. aspirin and indomethacin) constricts the DA in utero, which is why these drugs are contraindicated in pregnancy [21]. These drugs are effective in 
treating PDA in the postnatal state, with approximately $70 \%$ effectiveness in term infants [2224]. In low birthweight infants, however, failure of DA closure remains associated with elevated neonatal mortality rates [25]. Moreover, despite use of nonsteroidal anti-inflammatory drugs (i.e. ibuprofen) to achieve DA closure, none improve mortality, and these inhibitors are often associated with adverse renal and mesenteric effects [26]. In the infants in which it is most needed (babies $<1000 \mathrm{~g}$, gestation age 23-26 weeks) ibuprofen is relatively ineffective. Thus, new treatments for PDA are still needed.

Despite the effects of various vasoactive substances produced by endothelial cells, oxygen-induced changes in vasotone are intrinsic to the vasculature SMCs, and are only modulated by vasoactive compounds produced by the endothelium. Studies using endothelialdenuded DA have shown that these vessels maintain their constrictive responses to oxygen, suggesting that the mechanism of oxygen sensing is endothelium independent $[27,28]$.

\subsection{The Homeostatic Oxygen Sensing System}

Although all cell types are sensitive to severe hypoxia, specialized tissues throughout the body, which in aggregate make up the homeostatic oxygen sensing system (HOSS), are designed to sense small changes in airway oxygen levels and arterial pO2 within the physiological range and initiate changes in vascular tone or neurosecretion that optimize oxygen uptake and delivery[29]. The tissues within the HOSS elicit changes in respiration, vascular tone and neurosecretion in response to hypoxia in order to ensure adequate oxygen delivery throughout the body. Included in the HOSS are chromaffin cells of the fetal adrenal medulla, type 1 cells within the carotid body and neuroepithelial bodies within the lungs, all of which respond to hypoxia via exocytosis of neurotransmitters [30]. Also included in the HOSS are the SMC within 
the small pulmonary arteries, fetoplacental arteries, and ductus arteriosus, all of which respond to changes in pO2 through alterations in vascular tone $[1,29]$.

Tissues within the HOSS couple an upstream sensor of arterial oxygen, typically the mitochondrial ETC, to downstream effector ion channels, usually potassium and or calcium channels. The sensors within the mitochondria are thought to alter the redox status of the cell in response to changes in pO2 and create redox signaling molecules (ROS) capable of diffusing out of the organelle and traveling towards the cell membrane. At the membrane, redox modifications via reactions with ROS alter the activity of redox-sensitive potassium $\left(\mathrm{K}^{+}\right)$channels, causing changes in membrane polarization. In the SMC, redox modifications of these $\mathrm{K}^{+}$channels during normoxia initiate membrane depolarization, leading to the activation of $\mathrm{Ca}_{\mathrm{L}}$ channels and the initiation of SMC contraction through the actions of the myosin light chain (MLC).

\subsection{Ion channels as effectors of oxygen signaling}

The mechanism of SMC contraction within the DA involves the control of redox and voltage sensitive $\mathrm{K}^{+}$and $\mathrm{Ca}^{2+}$ channels. It is well established that the source of increasing cytosolic $\left[\mathrm{Ca}^{2+}\right]$ following SMC membrane depolarization is largely derived from the extracellular pool $[31,32]$. Injection of verapamil, an inhibitor of $\mathrm{Ca}_{\mathrm{L}}$ channels, immediately after caesarean delivery has been shown to inhibit the spontaneous constriction of the DA in newborn rats, suggesting a critical role for the $\mathrm{Ca}_{\mathrm{L}}$ channel in mediating SMC contraction within the DA [10].

In the adult pulmonary circulation, voltage-gated $\mathrm{K}^{+}$channel inhibition by tetraethylammonium (TEA) and 4-AP mimics hypoxic pulmonary vasoconstriction (HPV) [33]. The whole cell-patch clamp method has shown that a tonic outward potassium current $\left(\mathrm{I}_{\mathrm{k}}\right)$ within the pulmonary artery SMC (PASMC) prevents $\mathrm{Ca}_{\mathrm{L}}$ channels from opening, and that the $\mathrm{Kv}$ 
channel inhibitor 4-AP mimics the effects of hypoxia on $\mathrm{I}_{\mathrm{k}}$. Similarly, 4-AP is also capable of constricting the rabbit DA [27]. The similar responses of the DA and PA to Kv channel inhibition, combined with the opposing responses to hypoxia suggests that these channels themselves are not the sensors of $\mathrm{pO} 2$, but rather something proximal to them is sensing oxygen content and regulating channel gating.

$\mathrm{Kv}$ channels are tetrameric assemblies of identical or similar transmembrane $\alpha$-subunits with both a cytosolic $\mathrm{N}$ and C-termini. Although over ten families of Kv channels exist, the Shaker family (Kv1) is the most widely distributed and is implicated in several physiological processes, including oxygen sensing in the DA [8,34-36]. The Kv proteins contain multiple cysteine residues which are capable of being redox modified, affecting the ion channel open state probability and/or gating $[37,38]$. This may prove important if redox molecules such as ROS are believed to be the mediators of oxygen sensing. The heterotetrameric composition of a channel is also been shown to impact oxygen sensitivity. For example, PASMCs expressing Kv1.5 in combination with Kv1.2 have been shown to have increased sensitivity to oxygen in comparison to those expressing homomeric Kv1.5 channels [39].

\subsection{The role of mitochondrial dynamics in DA closure}

Recently, Hong et al. demonstrated that mitochondrial dynamics, the process of fission and fusion which dictate mitochondrial morphology, may also play an important role in oxygen sensing in the DA. Within five minutes of detecting an increased $\mathrm{pO} 2$, the mitochondria in DASMC begins to fragment [40]. This fragmentation results in increased oxidative metabolism and an increased production of mitochondrial derived ROS, namely hydrogen peroxide $\left(\mathrm{H}_{2} \mathrm{O}_{2}\right)$. Upon sensing a physiological increase in $\mathrm{pO} 2$, activation of the mitochondrial fission mediator, DRP1, occurs via post-translational modification. This active form of DRP1translocates to the 
mitochondrial outer membrane, where it is capable of forming a multimeric, ring-like structure around the organelle to facilitate fission [41]. Using DA from humans as well as full-term rabbits, DRP1 expression was measured under both hypoxic and normoxic conditions. Inhibition of DRP1 by the small molecule DRP1 GTPase inhibitors (e.g. Mdivi-1) [42] or molecular inhibition (e.g. small interfering RNA, siDRP1), was found to inhibit the constrictor response of the DA to normoxia, without altering its response to other vasoconstrictor stimuli, such as $\mathrm{KCl}$ and phenylephrine. This selective effect on normoxia-induced vasoconstriction mimics that seen with inhibitors of the ETC. However, mitochondrial fission precedes changes in $\mathrm{H}_{2} \mathrm{O}_{2}$ levels or vascular tone, suggesting that mitochondrial fission is an obligatory early step in oxygen-sensing in the DA [40].

\subsection{Reactive oxygen species as mediators of oxygen sensing}

In the mid 90s, Archer et al. demonstrated that changes in the redox status of the PA and aorta dictates SMC membrane polarization and contraction [43]. Specifically, it was found that PA rings incubated with anti-oxidants such as duroquinone or co-enzyme $\mathrm{Q}_{10}$ caused membrane depolarization and vasoconstriction. In contrast, incubation with the oxidant diamide caused membrane hyperpolarization and relaxation of constricted arterial rings. Both of these effects were observed in endothelium-denuded rings, suggesting that the effects of redox status on SMC contraction occurs in an endothelium-independent manner. Additionally, these findings suggest that changing the redox status of the SMC directly affects the whole-cell $\mathrm{K}^{+}$channel current. This may be possible due to the oxidation/reduction of key cysteine residues within the Kv subunits, which can alter the gating and open state probability of the $\mathrm{K}^{+}$channel [44].

Interestingly, Olschewski et al. demonstrated that the reducing agent DTT caused decreased $\mathrm{K}^{+}$ channel current, membrane depolarization, and vasoconstriction in the PA, while conversely 
increasing $\mathrm{K}^{+}$channel current and causing hyperpolarization and vasodilation in the DA [45]. These opposing effects suggest that reducing agents mimic hypoxia in both PA and DA.

The mechanism responsible for altered redox status during SMC oxygen sensing is thought to reside within the ETC of the mitochondria. As electrons flow through the inner mitochondrial membrane, a small percentage of electrons $(0.2-2 \%$ under normal physiological conditions) leak out of the ETC at several distinct sites [46-48]. Leaked electrons from complexes I and III combine with molecular oxygen to produce superoxide anion $\left(\mathrm{O}_{2}^{--}\right)$, a toxic form of ROS [49-51]. This anion is quickly converted by the actions of superoxide dismutase, into $\mathrm{H}_{2} \mathrm{O}_{2}$, a less toxic ROS with a larger diffusion radius [1,52]. Both superoxide anion and hydrogen peroxide are capable of oxidizing different classes of cellular targets. $\mathrm{H}_{2} \mathrm{O}_{2}$ is thought to play an important role as a signaling molecule due to its ability to oxidize thiol moieties on cysteine or methionine residues, causing structural and functional changes in target proteins through the formation of disulfide bridges [53]. In the context of SMC contraction, $\mathrm{H}_{2} \mathrm{O}_{2}$ may regulate membrane polarization by modulating the oxidative status of redox-sensitive $\mathrm{K}^{+}$ channels. In response to a shift from hypoxia to normoxia, the DA's mitochondria increase production of ROS, leading to oxidative modifications of ion channels to alter their activity and induce vasoconstriction. It is also worth noting that although ROS production by NADPH oxidase has been implicated in various oxygen sensing mechanisms, NADPH oxidase deficiency (chronic granulomatous disease) was not found to alter HPV in mice, and a specific role for NADPH oxidase in oxygen sensing in the DA has not been established [54, 55].

Although the role of the mitochondria in producing ROS is well established, how ROS production varies in response to hypoxia remains controversial and may be tissue specific. Despite various investigators reporting that ROS production varies in proportion to levels of 
oxygen in the blood [56-61], several groups have proposed that ROS production increases in response to hypoxia (in the PA) [62-68]. Although these contrasting findings have been attributed to differing detection methods used to measure ROS, an interesting find by Barneo et al. suggests that ROS production during hypoxia differs depending on the mitochondrial compartment measured, observing an increase in the intermembrane space (IMS) and decrease in the mitochondrial matrix in response to hypoxia in the carotid body [69]. Despite the controversy surrounding ROS production during hypoxia in the pulmonary vasculature, it is worth noting that there is no disagreement about ROS production decreasing during hypoxia which accompanies cardiac ischemia [70] or that ROS production increases with rising pO2 in the DA [71].

\subsection{Electron transport chain complexes as oxygen sensors}

Inhibitors of the ETC can alter ROS production by blocking electron transfer at specific points along the chain. Based on whether or not ROS is believed to increase or decrease in response to hypoxia, the explanation of complex inhibition on ROS generation differs. If ROS is believed to increase during hypoxia or after complex inhibition, this may be explained by a decreased flux of electrons along the ETC resulting in retrograde flow [69]. This retrograde flow may increase the chances of electron leakage out of the IMS, and therefore increase ROS production. If ROS is believed to decrease during hypoxia, this can be explained by an overall decrease in electron flux through the ETC following a decrease in oxidative metabolism.

Reduced overall electron flux would lead to reduced leakage, fewer radicals being produced, and decreased $\mathrm{H}_{2} \mathrm{O}_{2}$ production [72]. The similar effects of hypoxia and reducing agents on vascular tone in the DA and PA suggest that hypoxia is more of a reduced state; rather than a state of oxidation. 
Evidence suggests that rotenone and antimycin, inhibitors of complexes I and III, respectively, mimic hypoxia in the DA and PA, suggesting that the oxygen sensor in the vasculature resides in the mitochondria [71-74]. In addition to the effects of inhibition of proximal ETC complexes on the vasculature, rotenone has also been shown to mimic hypoxia in the carotid body, as evident by its ability to increase catecholamine secretion rate by the type 1 glomus cells $[69,75]$. Further implicating Complex I in oxygen sensing, fawn hooded rats (FHR), a spontaneously pulmonary hypertensive strain, show multiple mitochondrial abnormalities, including a decreased expression of Complex I [76]. These deficiencies result in reduced total ROS production as well as an inability to respond to changes in $\mathrm{pO}_{2}$ with proportionate changes in ROS. In other words, the FHR are in a state of pseudohypoxia in which mitochondrial ETC dysfunction mimics the redox environment of hypoxia, despite normal ambient PO2. FHRs also display a reduced vasoconstrictor response to acute hypoxia and rotenone, in comparison to wildtype rats, mimicking the diminished responses of normal rats exposed to chronic hypoxia [76].

A recent publication by Dunham-Snary et al. demonstrates that HPV is initiated by a $\mathrm{PO}_{2}$-dependent decrease in production of $\mathrm{H}_{2} \mathrm{O}_{2}$, reflecting hypoxic suppression of ROS production from Complex I [77]. Specifically, it was found that the Ndufs2 (NADH dehydrogenase [ubiquinone] iron-sulfur protein 2), a subunit within Complex 1 which is also the binding site for rotenone and ubiquinone, is critical in oxygen sensing in the PASMC. Downregulation of this subunit through the use of siRNA (siNdufs2) was found to mimic the effects of chronic hypoxia, as evident by changes in redox status (according to the redox theory of hypoxia): decreased production of $\mathrm{H}_{2} \mathrm{O}_{2}$ and increased ratio of NADH/NAD ${ }^{+}$. siNdufs 2 was also found to mimic the metabolic changes of authentic hypoxia, causing decreased oxygen 
consumption rate. siNDUFS2 selectively depressed HPV, diminishing the hypoxic rise in intracellular $\left[\mathrm{Ca}^{2+}\right]$ caused by exposing PASMC to hypoxic perfusate while maintaining the $\mathrm{KCl}$ induced increases in $\left[\mathrm{Ca}^{2+}\right]$. In partial agreement with these findings, Barneo et al. had earlier showed that Ndufs 2 is also critical to oxygen sensing within the type 1 cells of the carotid body $[69,78]$. Ndufs2 ablation in adult mice produced the progressive disappearance of the hypoxic ventilatory response and reduced the secretory response of carotid body glomus cells to acute hypoxia. As in our work, they found the effect of mice lacking Ndufs2 in their glomus cells was selective, evident in that the glomus cells still maintained normal responses to hypercapnia and hypoglycemia. In partial disagreement with our observations in PASMC, it was found that hypoxia increased ROS in the mitochondrial IMS compartment; although consistent with our findings they noted lack of Ndufs2 decreased ROS within the mitochondrial matrix [69]. It is unknown which compartment of the mitochondria and which ROS pool is critical to $\mathrm{O}_{2}$ sensing in the glomus cells, PASMC or DASMC.

In agreement with the importance of the proximal ETC (meaning complexes I-III) in oxygen sensing, Waypa et al. have shown that superoxide generation at Complex III is critical in PASMC hypoxic signaling [79]. The Rieske iron-sulfur protein (RISP) in Complex III is the catalytic subunit which facilitates the transfer of electrons from ubiquinol to cytochrome $\mathrm{c} 1$. This electron transfer forms ubisemiquinone, a free radical capable of forming superoxide anion by reacting with molecular oxygen $[80,81]$. They found that depletion of RISP in cultured PASMC abolished the hypoxia-induced ROS production within the mitochondrial IMS and cytosol, while cells maintained their ability to increase cytosolic $\left[\mathrm{Ca}^{2+}\right]$ in response to exogenous hydrogen peroxide, signaling that RISP deletion had no effect on the downstream signaling pathway. The results of this study are in agreement with the authors own prior work, showing that 
myxothiazol, a Complex III inhibitor of electron transfer to RISP, attenuated ROS production in response to hypoxia in cultured PA myocytes [82]. Their research disagrees with ours in that hypoxia increased ROS production, albeit they do concur with our earlier assessment that the sensor resides in the mitochondrial ETC. Although studies showing the importance of complexes I and III in oxygen sensing in the DA have been performed, the role of RISP vs Ndufs2 in DA oxygen signaling has not been explored [8].

\subsection{The role of Rho Kinase in DA closure}

The electrical phase of DASMC contraction occurs within 5-10 minutes of rising pO2, and is characterized by membrane depolarization and SMC constriction following the influx of calcium [83]. In contrast, sustained DASMC contraction is dependent on the regulation of the phosphorylation state of MLC [84]. The initiation of SMC contraction in the DA is caused by increased cytosolic $\left[\mathrm{Ca}^{2+}\right]$ activating $\mathrm{Ca}^{2+} /$ calmodulin-dependent MLC kinase, causing constriction through phosphorylation of MLC. The sustained contraction responsible for DA closure is caused by calcium sensitization, a process mediated by the actions of the Rho-kinase pathway, whereby constriction of the SMC occurs independently of ongoing calcium influx. The effects of this pathway result in MLC phosphatase (MLCP) inhibition, causing sustained constriction through persistent phosphorylation of MLC [84].

In studies on human and rabbit DA conducted by Kajimoto et al., Rho-kinase activation was found to be necessary for sustained oxygen induced SMC constriction, with prolonged exposure leading to an increased contribution of the Rho kinase pathway to DA constriction[83]. Additionally, persistent activity of Rho-kinase occurred due to a positive-feedback loop in fullterm, but not preterm rabbit DA. In term rabbit DA expression of key components of the Rho pathway are increased by Rho-kinase activity. Lastly, it was found that the activation of the Rho- 
kinase pathway was redox modulated, with increased $\mathrm{H}_{2} \mathrm{O}_{2}$ being capable of increasing ROCK-1 expression, a serine/threonine kinase which acts as the downstream effector during calcium sensitization. Thus, DA functional closure has at least two phases, an early mitochondrialregulated ion channel-mediated phase and a later rho-kinase mediated phase. 


\section{Chapter 3}

\section{Methodology}

Experimental Animals: All animal experiments conducted at Queen's University were done in accordance with the Canadian Council on Animal Care (CCAC) regulations and were approved by the Queen’s University Animal Care Committee (Protocol \#2017-1715).

Human DA tissues: DA samples from humans were harvested during congenital heart surgery at either the University of Chicago (IRB \#A3523-01) or the University of Nebraska (IRB \#10011-EP), after ethics approval from each institution. Ethics approval from Queen's University Health Sciences and Affiliated Teaching Hospitals Research Ethics Board (HSREB) (TRAQ \#6007784) was obtained prior to the use of human DASMC lines for ongoing research.

Tension measurements in term rabbit DA rings: Pregnant New Zealand White rabbits at 29 days gestation ( $\mathrm{n}=11, \mathrm{n}=29$ individual DA rings) were anaesthetized with $10 \mathrm{mg} / \mathrm{kg}$ ketamine and $0.2 \mathrm{mg} / \mathrm{kg}$ medetomidine. Rabbit kits were delivered via cesarean section and a midline sternotomy was performed immediately prior to the onset of respiration. The heart and lungs were excised en bloc and placed in hypoxic Krebs solution. The DA was dissected free from adventitia under a Scribolux 2.8x magnifier (Eschenbach, Danbury, CT, USA) and severed at its connections with the pulmonary trunk and the descending thoracic aorta. Within 5-10 minutes of harvesting the DAs, vessels were mounted on a force transducer using titanium wires at an experimentally-determined, optimal resting tension of $\sim 800 \mathrm{mg}$, in hypoxic Krebs $(\sim 40 \mathrm{mmHg}$ pO2, $\mathrm{pH}$ 7.35-7.45). After allowing the tissues to equilibrate for 30 minutes, tissues were exposed to varying pO2 levels $(35-155 \mathrm{mmHg})$. At peak oxygen induced constrictions, tissues 
were treated with either ETC leak suppressors S1QEL or S3QEL (1-100 $\mu \mathrm{M}$ in DMSO), ETC complex inhibitors rotenone (10 $\mu \mathrm{M}$ in DMSO) or antimycin $\mathrm{A}(10 \mu \mathrm{M}$ in EtOH) or vehicle controls (DMSO). These doses were determined by dose finding experiments to establish doses that had effects on DA tone greater than that of the vehicle, or based on previous research measuring the effects of ETC inhibitors on DA tone. In some experiments, electron leak suppressors were added prior to phenylephrine (PE) or $\mathrm{KCl}$ induced constriction events. At the end of each experiment, $80 \mathrm{mM} \mathrm{KCl}$ was administered. $10 \mu \mathrm{M}$ meclofenamate (an inhibitor of prostaglandin production) and $100 \mu \mathrm{M}$ L-Nitro arginine methyl ester (L-NAME, an inhibitor of nitric oxide production) were present in all ring bath experiments.

Isolation of DASMC: To establish rabbit and human DASMC lines, DA tissues were minced using a razor blade. Minced tissue was placed in $50 \mathrm{~mL}$ falcon tubes containing $2 \mathrm{~mL}$ of warm digestion buffer (RPMI with 2\% FBS, $13 \mathrm{WU} / \mathrm{mL}$ Liberase, and $100 \mathrm{mg} / \mathrm{mL}$ DNAase). Falcon tubes were vortexed for 20 seconds prior to incubation for 15 minutes at $37^{\circ} \mathrm{C}$ in an orbital shaker. Tissues were vortexed for 20 seconds, and incubated again for 15 minutes. Digested tissues were then filtered through a pre-wet $70 \mu \mathrm{M}$ cell strainer, and the filtered cell suspension was washed with $5 \mathrm{~mL}$ PBS. Cells were centrifuged at $1200 \mathrm{rpm}$ for 7 minutes at $21^{\circ} \mathrm{C}$. After centrifugation, the supernatant was aspirated off and the cell pellet was dissolved in $2 \mathrm{~mL}$ hypoxic M231 smooth muscle cell growth media (supplemented with 5\% smooth muscle growth supplement, $10 \%$ FBS, $1 \%$ L-glutamine, $1 \%$ penicillin/streptomycin, and $10 \mu \mathrm{g} / \mathrm{mL}$ ciprofloxacin $\mathrm{HCl})$, and was plated and finally cultured at $37^{\circ} \mathrm{C}$ in a hypoxic incubator $\left(2.5 \% \mathrm{O}_{2}\right.$ [40 mmHg $\mathrm{pO}_{2}$ ], $5 \% \mathrm{CO}_{2}$, balance $\mathrm{N}_{2}$ ). 
Establishing purified DASMC populations: To obtain a colony of purified DASMC, negative flow sorting experiments were performed on cells collected from DA tissues to exclude fibroblasts $\left(\mathrm{CD} 90^{+}\right)$and endothelial cells $\left(\mathrm{CD} 31^{+}\right)$. Cultured cells were trypsinized $(0.25 \%$ Trypsin-EDTA, Gibco), washed with PBS, and resuspended in $1 \mathrm{~mL}$ of hypoxic M231 with 4\% FBS. After counting, the cell suspensions were aliquoted into 4 Eppendorf tubes, two for singlestaining using PE anti-CD90/Thy1 (MRC OX-7, Abcam ab33694, Cambridge, MA, USA; $5 \mu \mathrm{L}$ per $10^{6}$ cells), and APC anti-CD31 (clone 390, BioLegend cat\#102409, San Diego, CA, USA; $1.25 \mu \mathrm{L}$ per $10^{6}$ cells), one for double staining using both previously mentioned antibodies, and one to serve as an unstained control. After adding staining solutions, all 4 Eppendorf tubes were vortexed and incubated for 30 minutes at $37^{\circ} \mathrm{C}$ in a hypoxic incubator. Cells were then centrifuged and resuspended in $1 \mathrm{~mL}$ PBS to wash away excess antibodies. Cells were sorted using the SH800S cell sorter (Sony Biotechnology, San Jose, CA, USA) to only include CD90 CD31 ${ }^{-}$cells and single stained and unstained cells were used to set up sorting and compensation parameters. After sorting, cells were collected in hypoxic M231 supplemented media and were plated and cultured at $37^{\circ} \mathrm{C}$ in a hypoxic incubator.

Mitochondrial metabolism measurements: Oxygen consumption rates (OCR) and extracellular acidification rates (ECAR) were measured simultaneously using an XF24 extracellular flux analyzer (Seahorse Bioscience, CA). A day prior to performing the experiment, rabbit DASMC were plated at an experimentally-determined, optimal density in a 24-well XF24 cell culture microplate (part \#100777-004), and an extracellular flux assay kit (part \#100850-001) was hydrated by loading $1 \mathrm{~mL}$ of XF calibrant solution (part \#100840-000) into each well in the utility plate and incubating at $37^{\circ} \mathrm{C}$ overnight in $\mathrm{CO}_{2}$ free incubator. The next day, 
measurements were taken after equilibration in XF assay media (part \#102353-100) supplemented with $4.5 \mathrm{~g} / \mathrm{L}$ glucose, with $\mathrm{pH}$ adjusted to 7.4 at $37^{\circ} \mathrm{C}$. To measure changes in OCR and ECAR after the addition of S1QEL (SML1948, Sigma-Aldrich, ON, CA) or S3QEL (SML1554, Sigma-Aldrich, ON, CA), electron leak suppressors or vehicle control (DMSO) were added to port A across the entire extracellular flux assay kit. Oligomycin, carbonyl cyanide-4 (trifluoromethoxy) phenylhydrazone (FCCP), and rotenone/antimycin A were added to ports B, $\mathrm{C}$, and $\mathrm{D}$, at 10X the working concentration. After equilibrating the extracellular flux assay kit in the XF24 analyzer, the hydrating cartridge was replaced with the cell culture microplate. For each phase of the experiment (baseline, intervention, oligomycin, FCCP, and rotenone/antimycin A) 3 measurement cycles were performed, consisting of 3-minute mix periods, 2-minute wait periods, and 3-minute measure periods. For all experiments, cells were challenged with $1 \mu \mathrm{M}$ oligomycin (to inhibit ATP synthase), $1 \mu \mathrm{M}$ FCCP (an uncoupling agent which maximizes OCR), and $1 \mu \mathrm{M}$ of rotenone/antimycin A (to inhibit mitochondrial respiration).

Measuring ROS production in DASMC: hDASMC were plated at an experimentallydetermined, optimal density for fluorescence imaging in a $\mu$-Slide 8 Well Glass Bottom plate (ibidi GmbH, part \#80827, Fitchburg, WI, USA). The next day, cells were stained with $10 \mu \mathrm{M}$ of MitoROS 580 dye using the Mitochondrial Superoxide Detection Kit (Abcam ab219943, Cambridge, MA, USA) for 30 minutes at $37^{\circ} \mathrm{C}$ in a hypoxic incubator, in the presence of either DMSO, $50 \mu \mathrm{M}$ S1QEL, $100 \mu \mathrm{M}$ S3QEL, or $10 \mu \mathrm{M}$ rotenone. After staining cells were imaged using an OkoLab stage-top microscope incubator (OkoLab Bold Line, Pozzuoli, Italy) and imaging was performed using a Leica TCS SP8 X confocal microscope (Leica Microsystems, Wetzlar, Germany). Cells were allowed to equilibrate in hypoxia $\left(2.5 \% \mathrm{O}_{2}, 5 \% \mathrm{CO}_{2}\right.$, balance $\left.\mathrm{N}_{2}\right)$ 
for 10 minutes, prior to imaging for 10 minutes in hypoxia followed by 10 minutes of normoxia (room air), capturing one frame every 12 seconds. Images were obtained and analyzed using either LAS-X software (Leica) or Fiji (ImageJ). For each treatment group, regions of interest (ROI) were drawn around 5 cells to track changes in MitoROS fluorescence over time. To compare changes in MitoROS fluorescence induced by increased oxygen content, ROI intensity over the last 4 images were compared to the initial 4 images taken during hypoxia.

Micro-CT imaging of DA in fetal kits: Pregnant New Zealand White rabbits at 29 days gestation ( $\mathrm{n}=7, \mathrm{n}=15$ individual kits) were anaesthetized with $10 \mathrm{mg} / \mathrm{kg}$ ketamine and $0.2 \mathrm{mg} / \mathrm{kg}$ medetomidine and rabbit kits were delivered via cesarean section. Rabbit kits were divided into three treatment groups: A) hypoxia, B) normoxia, and C) normoxia + S1QEL. Upon removal from the amniotic sac, kits were either placed in a $100 \% \mathrm{O}_{2}$ chamber for 20 minutes before being anesthetized with ketamine (groups B and C) or immediately anesthetized with ketamine (group A), before their first breath. Rabbit kits were placed in lateral decubitus and a left thoracotomy was performed to prevent bleeding via the umbilical vasculature. A right femoral arteriotomy was also performed to permit the exit of blood and perfusion solution. Three luer lock syringes were carefully filled with heparinized PBS (10 units; $2 \mathrm{~mL}), 4 \%$ PFA ( $8 \mathrm{~mL})$, and the contrast agent iohexol $300 \mathrm{mgI} / \mathrm{mL}$ (Omnipaque with $3 \%$ gelatin, $8 \mathrm{~mL}$ ) to avoid bubbles, and were capped and kept at $37^{\circ} \mathrm{C}$ prior to use. A 25 gauge butterfly catheter was inserted in the LV a distance no further than $1.5 \mathrm{~mm}$ while the heart was beating. The catheter was then connected to the solutions described above. The perfusion was performed at a constant rate of $2 \mathrm{~mL} /$ minute (controlled by hand), as follows: $250 \mu \mathrm{L}$ S1QEL ( $100 \mu \mathrm{M}$, group C only), heparinized PBS, 4\% PFA, and Omnipaque with 3\% gelatin. After perfusion, rabbit kits were positioned on ice with 
their heads elevated at a $45^{\circ}$ angle to the table to prevent leak of the contrast agent, and transferred to the imaging lab in the Queen's CardioPulmonary Unit. The micro-CT images were acquired exploiting VECTor ${ }^{4} \mathrm{CT}$ pre-clinical scanner (MILabs B.V., Utrecht, Netherlands) equipped with a cone-beam X-ray CT system. The X-ray source rotates around a fixed bed allowing the rabbit kit to be kept in the horizontal position in the scanner. The measurement was carried out at an acceleration voltage of $50 \mathrm{kVp}$ and an X-ray tube current of $430 \mu \mathrm{m}$ in an ultrafocused mode to take 3,600 projections over a $360^{\circ}$ scan with an exposure time of $40 \mathrm{~ms}$. The tomographic CT images were reconstructed using MILabs reconstruction software using Hann projection with a filter of a 40-mm voxel grid to generate a 3D CT image. Reconstructed slice data and maximum intensity projection (MIP) images were either processed using PMOD 3.9 software (PMOD Technologies Ltd., Zurich, Switzerland) and were manually converted to DICOM files for 3D rendering using OsiriX Lite software.

Statistical Analysis: Values are expressed as the mean \pm SEM. For normally distributed data, values are shown as the mean $\pm \mathrm{SEM}$, and Student's t-test or ANOVA were used to assess intergroup differences. P values $<0.05$ were considered statistically significant. 


\section{Chapter 4}

\section{Results}

Tension measurements in rabbit DA rings: Exposure to normoxia caused an average $1100 \pm 62$ mg increase in DA tension. Exposure to S1QEL at concentrations of $10 \mu \mathrm{M}, 50 \mu \mathrm{M}$, or $100 \mu \mathrm{M}$ caused stepwise decreases in DA tension (Figure 2A), in contrast with S3QEL which caused no significant change in DA tension at concentrations of $10 \mu \mathrm{M}, 50 \mu \mathrm{M}$, or $100 \mu \mathrm{M}$ (Figure 2B). 50 $\mu \mathrm{M}$ S1QEL caused an average $25.3 \pm 17.6 \%$ reduction in DA tension $(* \mathrm{p}<0.05, \mathrm{n}=8)$ whereas $100 \mu \mathrm{M}$ S3QEL only reduced DA tension by $4.4 \pm 2.2 \%(\mathrm{n}=4)$ (Figure $2 \mathrm{C}) .10 \mu \mathrm{M}$ of Complex I inhibitor rotenone reduced DA tension an average of $11.4 \pm 12.4 \%(\mathrm{n}=5)$, and $10 \mu \mathrm{M}$ of Complex III inhibitor antimycin A reduced DA tension an average $21.0 \pm 16.2 \%(n=7)$, while vehicle (DMSO) had minimal effect (change in tension $-2.6 \pm 1.5 \%, \mathrm{n}=6$ ). $50 \mu \mathrm{M}$ S1QEL had no significant effect on PE $(10 \mu \mathrm{M})$-induced constriction $(1.1 \pm 0.1 \%, \mathrm{n}=5)$ compared to vehicle (DMSO) $(1.0 \pm 0.1 \%, \mathrm{n}=3)$. The change relative to initial PE response was calculated by dividing the magnitude of the PE response in the presence of S1QEL or vehicle by the initial PE response in the absence of any intervention (Figure 2D). This data suggests that electron leak from Complex I site $\mathrm{I}_{\mathrm{Q}}$, but not Complex III site $\mathrm{III}_{\mathrm{Q}}$, is implicated in DA oxygen sensing. 
A)

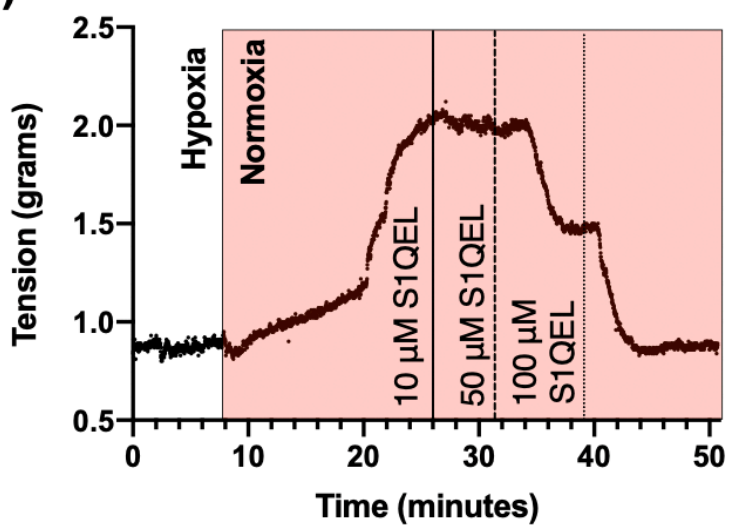

C)

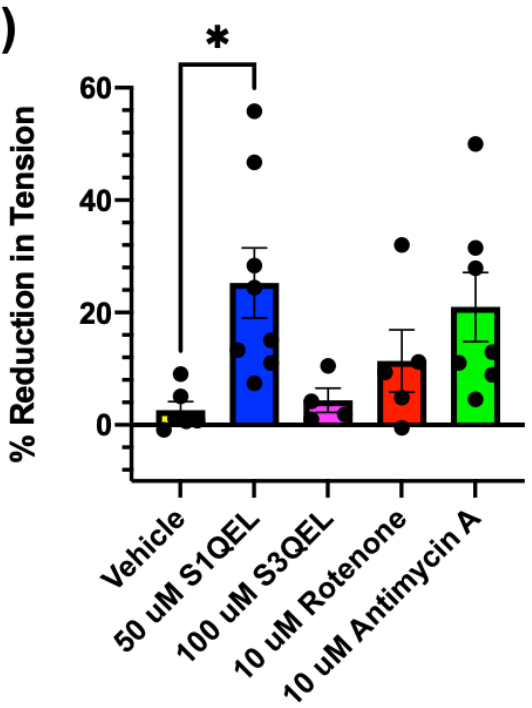

B)

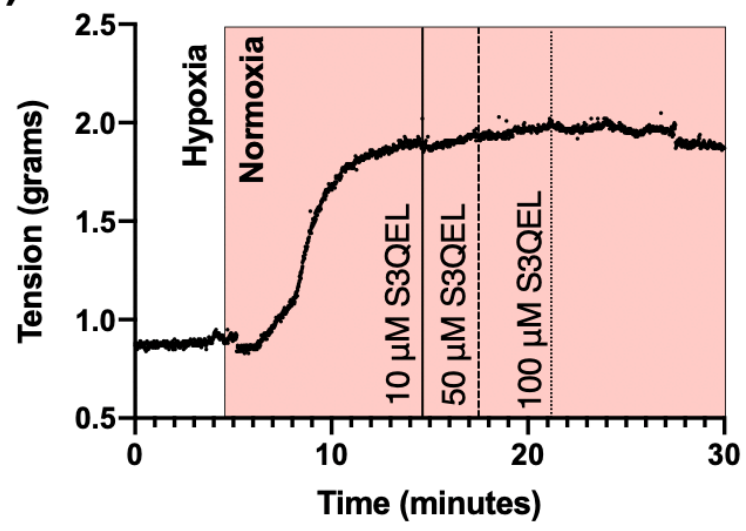

D)

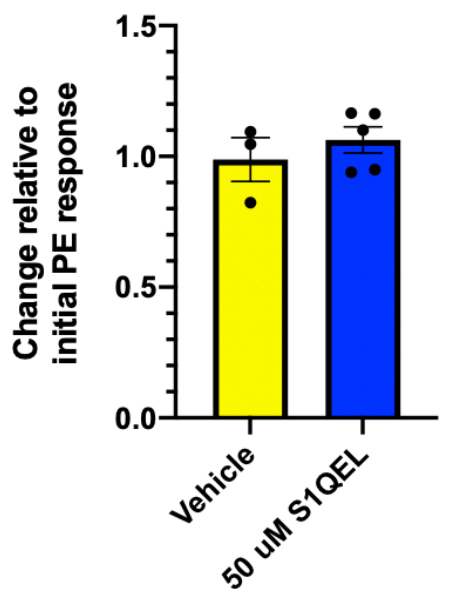

Figure 2 Effects of electron leak suppressors and ETC complex inhibitors on DA vessel tone. In panels (A) and (B), DA tension was measured using a force transducer, and constriction was induced after switching from hypoxia ( $35 \mathrm{mmHg} \mathrm{pO2})$ to normoxia ( 155 mmHg pO2, red box). In panels $(\boldsymbol{A})$ and $(\boldsymbol{B})$, dose responses of constricted DA to $10 \mu M, 50 \mu M$, and $100 \mu M$ SIQEL $(\boldsymbol{A})$ and S3QEL $(\boldsymbol{B})$ are shown. In panel $(\boldsymbol{C})$, percent reduction in DA tension 5 minutes after the addition of vehicle (DMSO, yellow), $50 \mu M$ SIQEL (blue), $100 \mu M$ S3QEL (magenta), $10 \mu M$ rotenone (red), or $10 \mu M$ antimycin A (green) is shown. In panel (D), DAs in hypoxia were exposed to $10 \mu M$ phenylephrine (PE) to induce constriction. After relaxing fully, DAs were exposed to $10 \mu M$ PE again in the presence of vehicle (DMSO, yellow) or $50 \mu M$ SIQEL (blue). * $p<0.05$.

Isolation of DASMC: General scheme for isolation of DASMC in both rabbit and human cells is shown in Figure 3. Unstained cells and single stained-cells were used to set up sorting and compensation parameters. Cells were first gated to identify live cells by their forward scatter (FSC) and side scatter (SSC) characteristics. Cells were then negatively sorted using antibodies against CD90 and CD31, to exclude fibroblasts and endothelial cells, respectively. 
A)

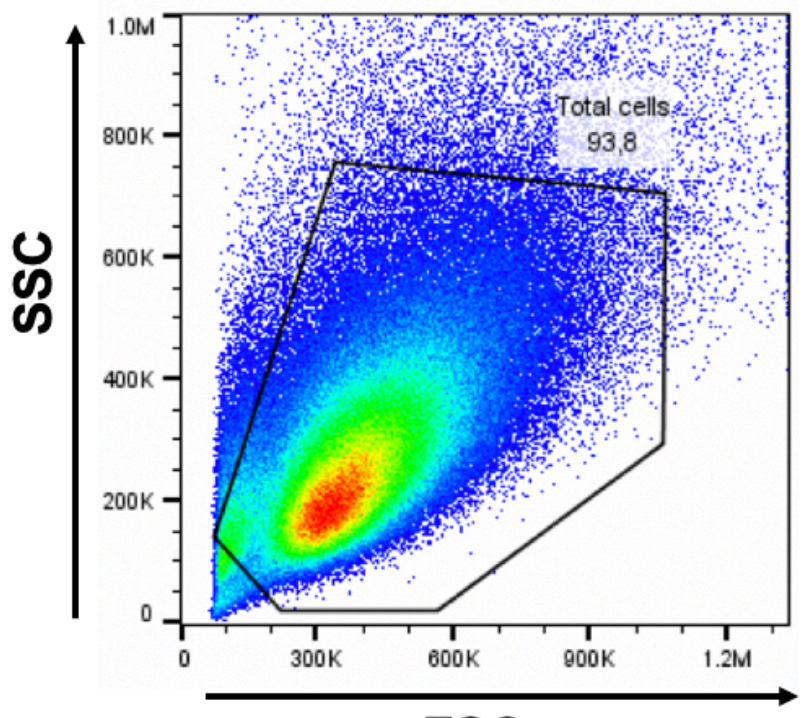

FSC
B)

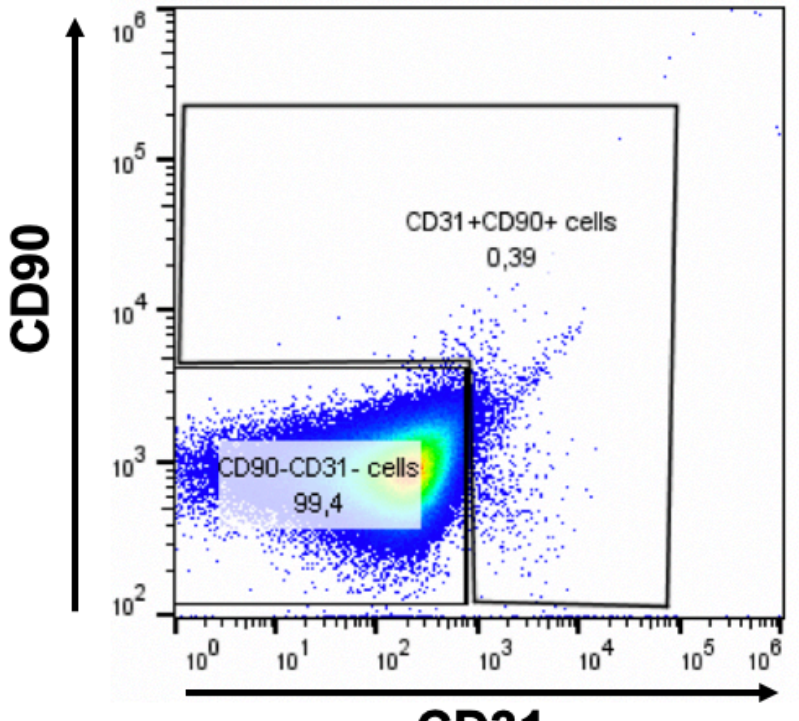

CD31

Figure 3 Isolation of DASMC using flow cytometry. A gating strategy was used to identify live cells by forward scatter (FSC) and side scatter (SSC) (A), and DASMC were isolated by negatively sorting for CD90 and CD31 to remove fibroblasts and endothelial cells, respectively $(\boldsymbol{B})$.

Mitochondrial metabolism measurements: Mitochondrial respiratory parameters were calculated according to the general scheme shown in Figure 4A, and changes in OCR values, measured in $\mathrm{pmol} \mathrm{O}_{2} / \mathrm{min} / \mu \mathrm{g}$ (normalized to total protein content in each well), are shown in Figure 4B-G. Acute response values calculated by measuring the change in OCR values after the addition of vehicle, electron leak suppressor or ETC inhibitor. Vehicle (DMSO) caused a slight increase in OCR values $(0.025 \pm 0.2, n=8)$, compared to decreased OCR rates observed after acute injection of $1 \mu \mathrm{M}$ S1QEL $(-0.3 \pm 0.39, \mathrm{n}=6), 10 \mu \mathrm{M}$ S1QEL $\left(-0.8 \pm 0.4, \mathrm{n}=8,{ }^{* *} \mathrm{p}<0.01\right), 1$ $\mu \mathrm{M}$ rotenone $\left(-1.9 \pm 0.8, \mathrm{n}=6,{ }^{* *} \mathrm{p}<0.01\right)$, and $10 \mu \mathrm{M} \mathrm{S} 3 \mathrm{QEL}(-0.5 \pm 0.8, \mathrm{n}=4)$ (Figure 4B). Maximal respiration values were calculated by subtracting the non-mitochondrial oxygen consumption measurements from the OCR values after addition of FCCP. DASMC treated with vehicle (DMSO) showed significantly higher maximal respiration values after exposure to FCCP $(5.8 \pm 2.5, \mathrm{n}=8)$, compared to cells treated with $1 \mu \mathrm{M}$ S1QEL $(2.7 \pm 0.8, \mathrm{n}=6), 10 \mu \mathrm{M}$ S1QEL 
$\left(3.1 \pm 0.9, \mathrm{n}=8,{ }^{*} \mathrm{p}<0.05\right), 1 \mu \mathrm{M}$ rotenone $\left(0.18 \pm 0.08, \mathrm{n}=6,{ }^{* *} \mathrm{p}<0.01\right)$, and $10 \mu \mathrm{M}$ S3QEL

$(4.4 \pm 3.6, n=4)$ (Figure 4C). Spare respiratory capacity values were calculated by subtracting the baseline OCR values from the maximal OCR values observed after the addition of FCCP. The spare respiratory capacity of DASMC treated with vehicle (DMSO) was higher $(3.3 \pm 1.6, n=8)$, compared to cells treated with $1 \mu \mathrm{M}$ S1QEL $(1.3 \pm 0.4, \mathrm{n}=6), 10 \mu \mathrm{M}$ S1QEL $(1.4 \pm 0.3, \mathrm{n}=8), 1$ $\mu \mathrm{M}$ rotenone $\left(0.02 \pm 0.04, \mathrm{n}=6,{ }^{* *} \mathrm{p}<0.01\right)$, and $10 \mu \mathrm{M}$ S3QEL $(2.2 \pm 2.4, \mathrm{n}=4)$ (Figure 4D). Nonmitochondrial oxygen consumption, or the remaining OCR observed after the addition of antimycin A and rotenone, was higher $(1.5 \pm 0.5, \mathrm{n}=8)$ in DASMC treated with vehicle (DMSO), compared to cells treated with $1 \mu \mathrm{M}$ S1QEL $(0.8 \pm 0.3, \mathrm{n}=6), 10 \mu \mathrm{M}$ S1QEL $\left(0.9 \pm 0.4, \mathrm{n}=8,{ }^{*}\right.$ $\mathrm{p}<0.05), 1 \mu \mathrm{M}$ rotenone $(1.3 \pm 0.4, \mathrm{n}=6)$, and $10 \mu \mathrm{M} \mathrm{S3QEL}(1.1 \pm 0.5, \mathrm{n}=4)($ Figure $4 \mathrm{E})$. ATP production values were calculated by subtracting the OCR values observed after the addition of oligomycin from the OCR values observed at baseline. ATP production was higher in DASMC treated with vehicle (DMSO) $(1.9 \pm 0.7, \mathrm{n}=8)$, compared to cells treated with $1 \mu \mathrm{M}$ S1QEL $\left(1.2 \pm 0.7, \mathrm{n}=6,{ }^{*} \mathrm{p}<0.05\right), 10 \mu \mathrm{M}$ S1QEL $\left(1.1 \pm 0.6, \mathrm{n}=8,{ }^{* *} \mathrm{p}<0.01\right), 1 \mu \mathrm{M}$ rotenone $(0.1 \pm 0.09$ $\left.\mathrm{n}=6,{ }^{* *} \mathrm{p}<0.01\right)$, and $10 \mu \mathrm{M}$ S3QEL $(1.9 \pm 1.1, \mathrm{n}=4)$ (Figure 4F). Proton leak values were calculated by subtracting non-mitochondrial oxygen consumption from OCR values observed after the addition of oligomycin. Proton leak in DASMC treated with vehicle (DMSO) $(0.5 \pm 0.2$, $\mathrm{n}=8)$ was similar to cells treated with $1 \mu \mathrm{M} \mathrm{S} 1 \mathrm{QEL}(0.4 \pm 0.2, \mathrm{n}=6), 10 \mu \mathrm{M}$ S1QEL $(0.6 \pm 0.2$, $\mathrm{n}=8)$, and $10 \mu \mathrm{M} \mathrm{S} 3 \mathrm{QEL}(0.5 \pm 0.4, \mathrm{n}=4)$, but higher than cells treated with $1 \mu \mathrm{M}$ rotenone $\left(0.3 \pm 0.1, \mathrm{n}=6,{ }^{*} \mathrm{p}<0.05\right)$ (Figure 4G). These results suggest that rotenone, but not S1QEL, impairs key mitochondrial respiratory parameters in DASMC, such as ATP production and spare respiratory capacity. Representative OCR tracings for rbDASMC measured using Seahorse XF Mito Stress Test are shown in Figure 5A-B, along with their corresponding ECAR tracings in 
Figure 5C-D. Taken together with our ex vivo work, these data suggest that S1QEL reverses ductal constriction through mechanisms unrelated to mitochondrial metabolism.
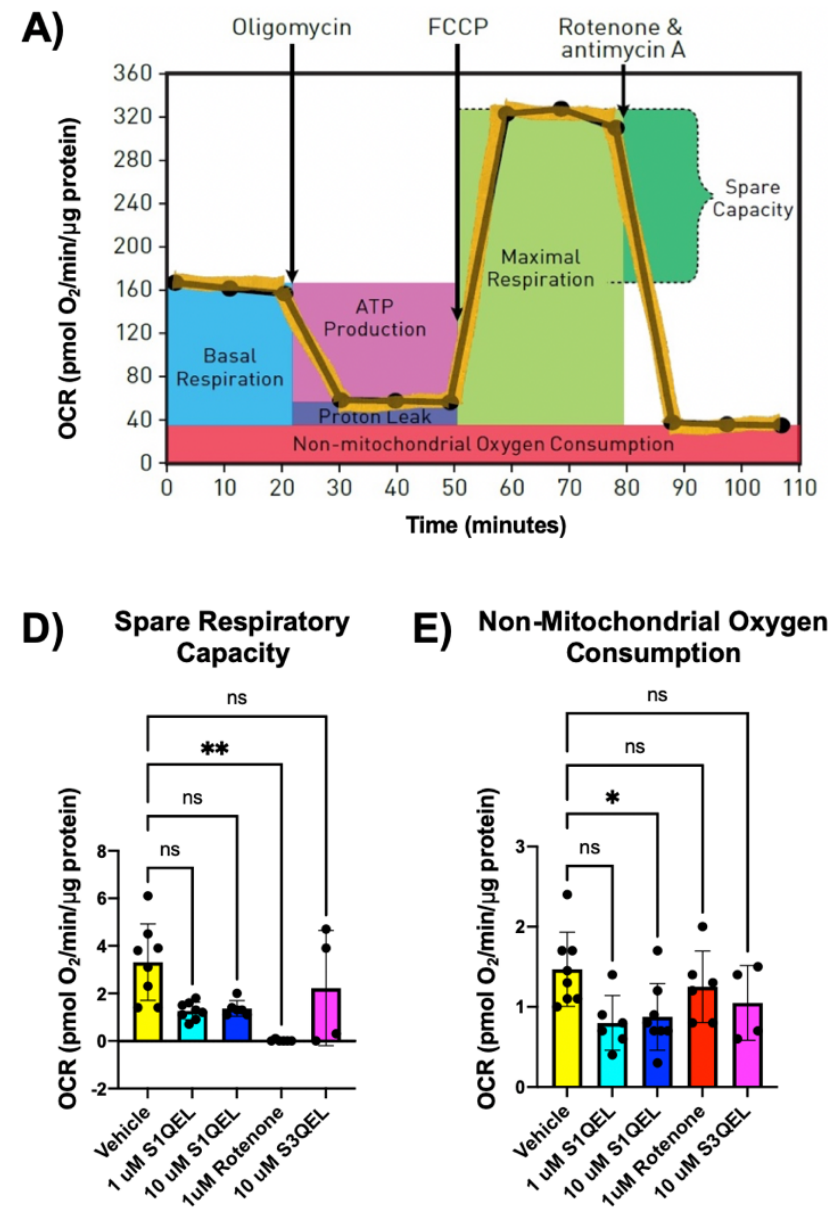

E) Non-Mitochondrial Oxygen

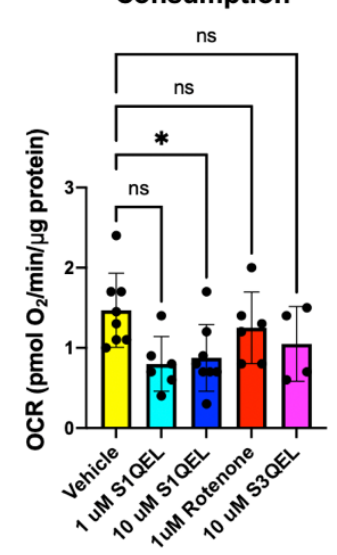

B)

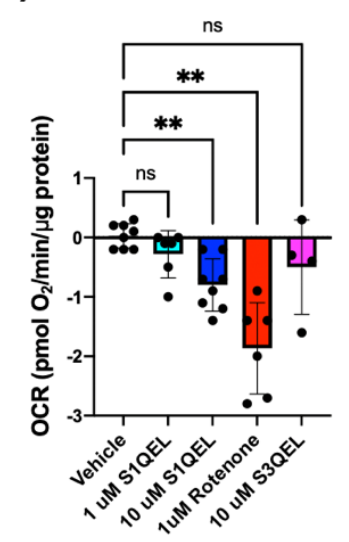

F)

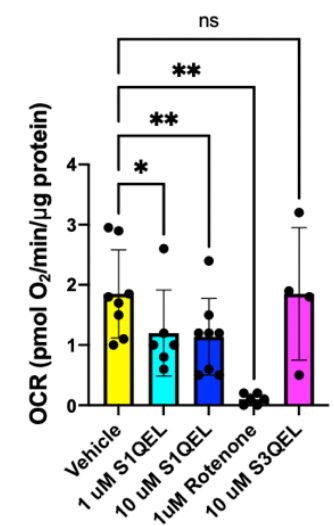

C)

Maximal Respiration

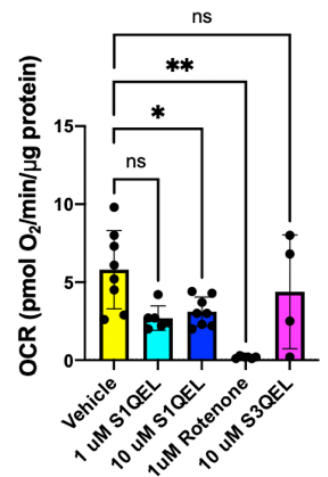

G)

Proton Leak

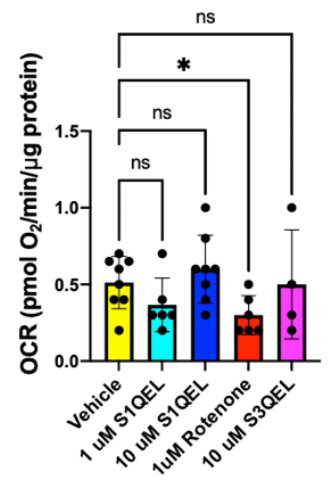

Figure 4 Impact of electron leak suppressors and rotenone on mitochondrial respiratory parameters. Panel (A) illustrates how various respiratory parameters are derived using the OCR tracings from the SeaHorse XF Mito Stress Test (shown in Figure $4 A / B)$. In panels (B) through $(\boldsymbol{G})$, mitochondrial respiratory parameters were compiled across eight different rbDASMC lines for cells exposed to vehicle (DMSO, yellow), $1 \mu \mathrm{M}$ SIQEL (cyan) or $10 \mu \mathrm{M}$ SIQEL (blue)), $10 \mu \mathrm{M}$ S3QEL (magenta), or $1 \mu \mathrm{M}$ rotenone (red). Data was normalized to total protein within each well. *,** $p<0.05$ and $p<0.01$, respectively. 

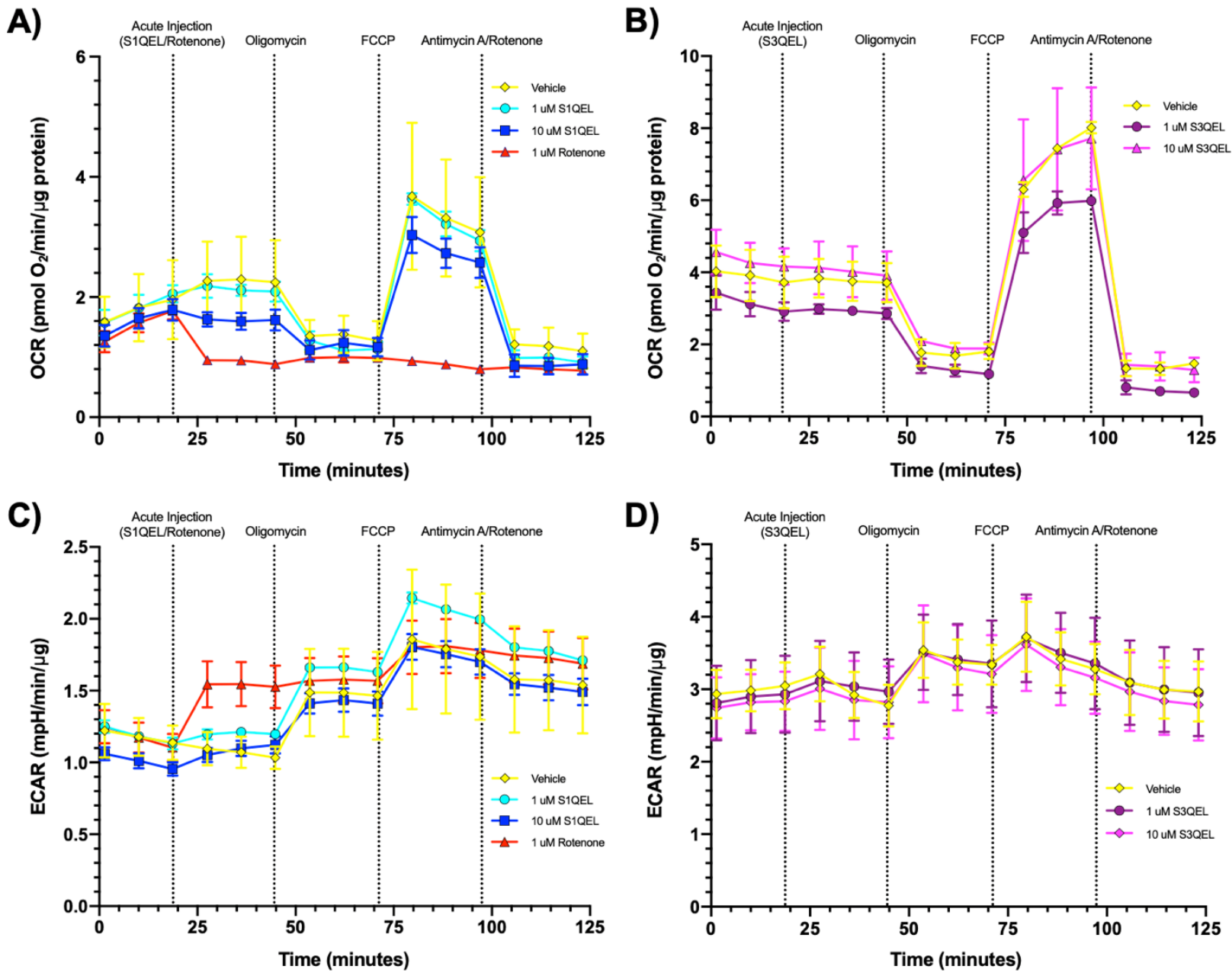

Figure 5 Impact of electron leak suppressors and rotenone on mitochondrial respiration using the SeaHorse XF Mito Stress Test. In panels $(\boldsymbol{A})$ and $(\boldsymbol{B})$, mitochondrial respiration is shown through measurement of oxygen consumption rates (OCR) measured in rabbit DASMC (rbDASMC). Cells were first exposed to an acute injection of either vehicle (DMSO, yellow), SIQEL (1 $\mu M$ (cyan) or $10 \mu M$ (blue), (A)) or rotenone (10 $\mu M$ (red), (A)), or S3QEL (1 $\mu M$ (purple) or $10 \mu M$ (magenta), (B)), followed by 1 $\mu M$ oligomycin, $1 \mu M F C C P$, and $1 \mu M$ of both rotenone and antimycin A. In panels $(C)$ and $(\boldsymbol{D})$, the extracellular acidification rates (ECAR) for the same cell lines measured in panels $(\boldsymbol{A})$ and $(\boldsymbol{B})$ are shown, following the same injection protocols.

ROS production during normoxia in DASMC: Representative images of fluorescence imaging experiments performed in hDASMC using MitoROS 580 are shown in Figure 6A/B. Increased superoxide production can be observed qualitatively after switching from hypoxia (Figure 6A) to normoxia (Figure 6B), with fluorescence intensity corresponding to superoxide production, on a scale of purple (low) to yellow (high). Figure 6C shows a representative tracing of fluorescence intensity for $6 \mathrm{hDASMC}$ (corresponding to individual ROI) during 10 minutes of hypoxia (light blue) and 10 minutes of normoxia (light red). MitoROS fluorescence (RFU) can 
be observed to increase after switching to normoxia. Four hDASMC cell lines were stained with MitoROS 580 (UC2, UC3, UC4, UC6) and their increase in fluorescence intensity after switching from hypoxia to normoxia was quantified (Figure 7A/B). Change in fluorescence intensity relative to hypoxic baseline was calculated using the average fluorescence intensity across the final four data points (end of normoxic period) obtained in the tracings shown in Figure 6C, and dividing these by the average fluorescence intensity across the first four data points (beginning of hypoxic period). hDASMC treated with vehicle (DMSO) showed larger increase in fluorescence intensity after exposure to normoxia $(1.4 \pm 0.1, \mathrm{n}=4)$, compared to $50 \mu \mathrm{M}$ S1QEL $(1.2 \pm 0.03, \mathrm{n}=4), 10 \mu \mathrm{M}$ rotenone $(1.2 \pm 0.2, \mathrm{n}=4)$, and $100 \mu \mathrm{M}$ S3QEL $(1.3 \pm 0.09, \mathrm{n}=4)$, indicating the leak suppressor reduced ROS production in the DAMSC. These results suggest that the electron leak suppressor S1QEL reduces DA tone ex vivo by decreasing ROS production within the tissue in response to normoxia. 


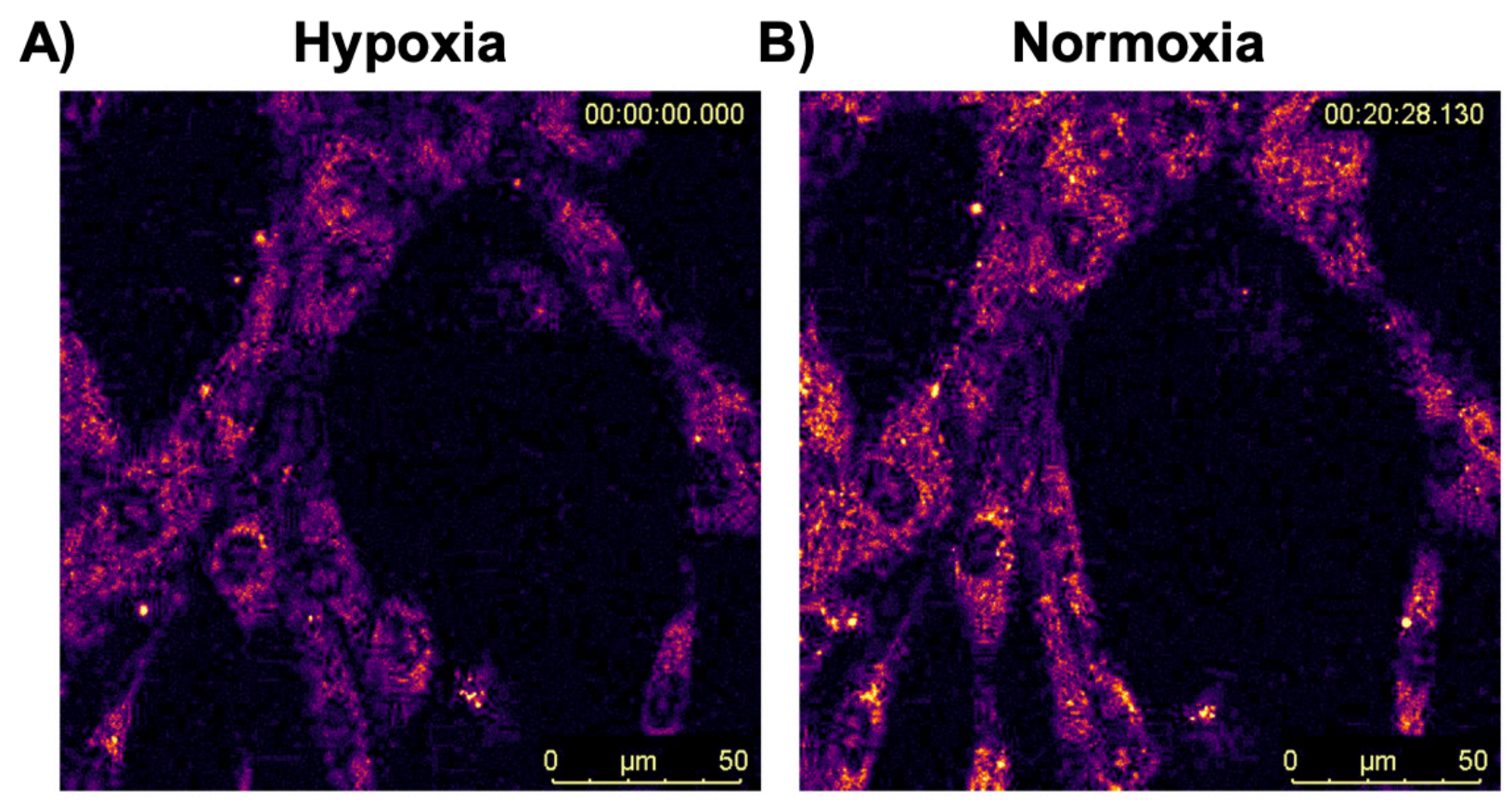

C)

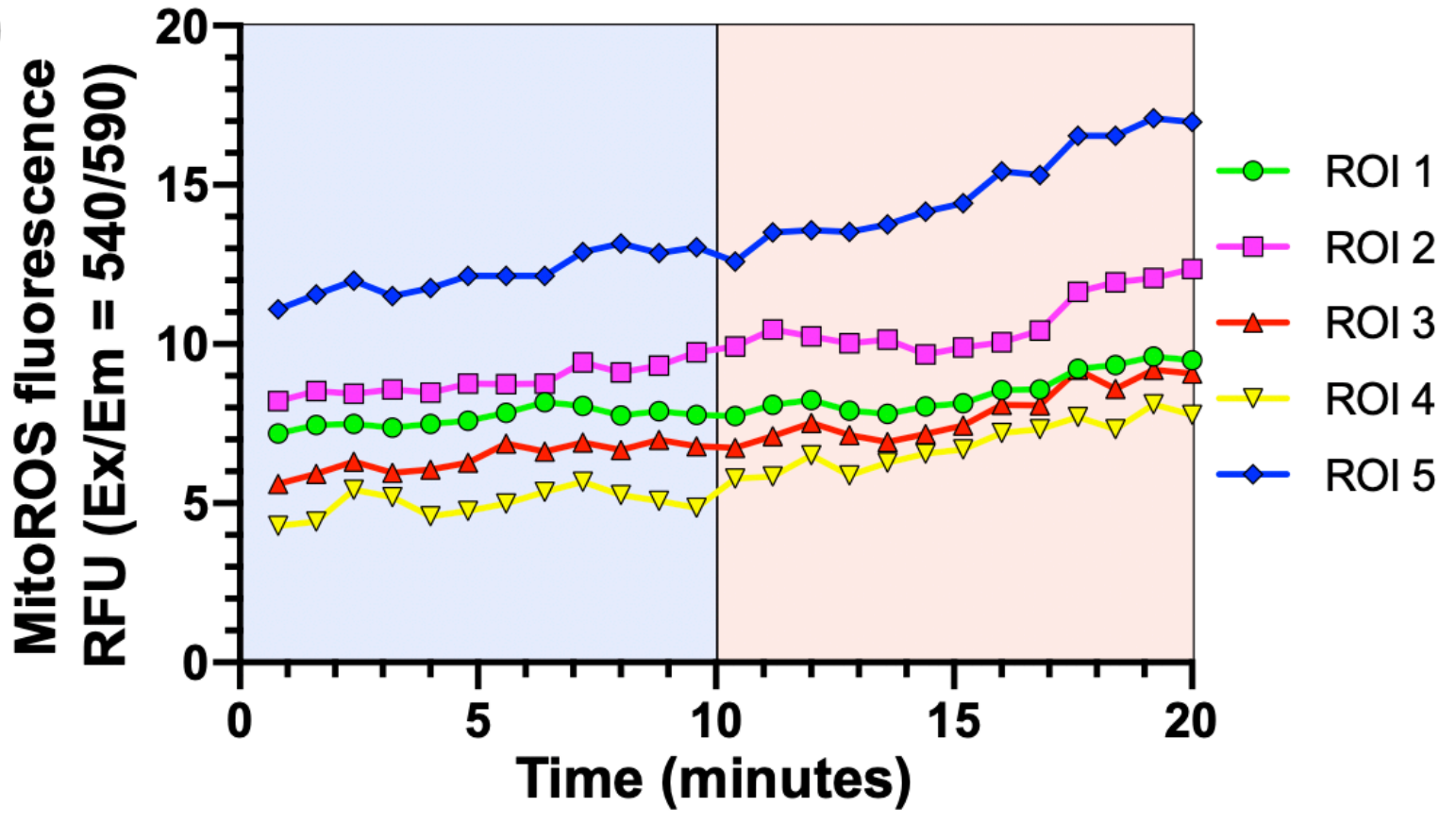

Figure 6 Superoxide measurement in hDASMC using MitoROS 580. Fluorescence intensity is depicted on a scale from purple (low superoxide production) to yellow (high superoxide production) in hDASMC immediately upon beginning imaging in hypoxia $(2.5 \%$ pO2) (A) or after 10 minutes of exposure to normoxia (room air) (B). Regions of interest (ROI) were drawn around individual cells, and their fluorescence intensity measured in relative fluorescence units (RFU) was measured over the course of the experiment $(\boldsymbol{C})$, with hypoxic and normoxic periods shown in blue and red, respectively. 
A)

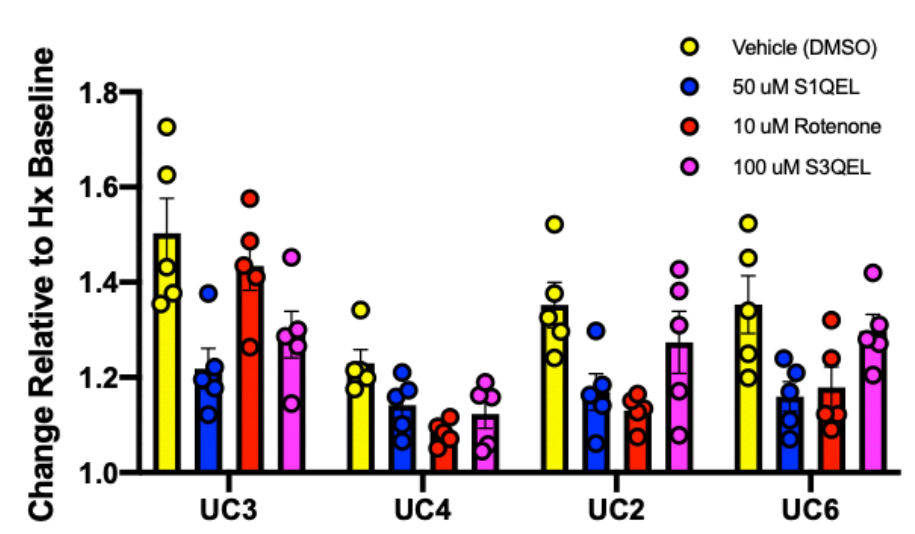

B)

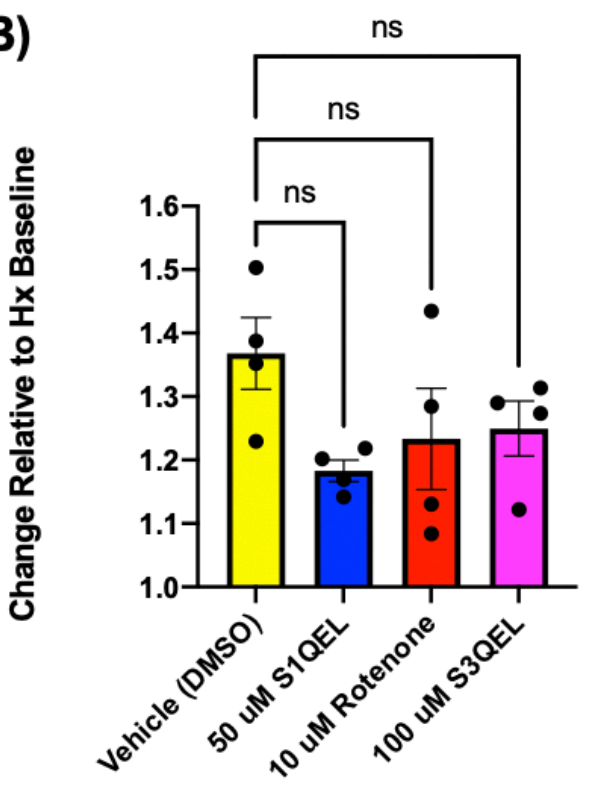

Figure 7 Effects of electron leak suppressors and rotenone on normoxia induced superoxide production. (A) Across four different hDASMC lines (UC2, UC3, UC4, and UC6), MitoROS fluorescence was compared between periods of normoxia and hypoxia, in cells exposed to vehicle (DMSO, yellow), $50 \mu \mathrm{M}$ S1QEL (blue), $100 \mu \mathrm{M} \mathrm{S3QEL} \mathrm{(magenta),} \mathrm{or} 10 \mu \mathrm{M}$ rotenone (red). Individual data points represent individual cells (ROI). (B) Data obtained from all four hDASMC lines were compiled together to analyze for intergroup differences using a one-way ANOVA.

Micro-CT imaging of DA in fetal kits: Representative MIP images of rabbit kits perfused with Omnipaque (with 3\% gelatin) immediately after cesarean delivery (hypoxia) or after exposure to $100 \% \mathrm{O}_{2}$ for 20 minutes in the presence of vehicle (normoxia) or $100 \mu \mathrm{M} \mathrm{S} 1 \mathrm{QEL}$ (normoxia + S1QEL) are shown in Figure 8A. Rabbit kits perfused immediately following delivery show complete ductal patency $(97.8 \pm 9.3 \%, \mathrm{n}=5)$, comparable to kits exposed to normoxia with 100 $\mu \mathrm{M}$ S1QEL $(80.9 \pm 15.5 \%, \mathrm{n}=5)$ whereas kits exposed to normoxia with vehicle (DMSO) treatment showed complete absence of their DA $(\mathrm{n}=5)$ (Figure 8B). Similarly, the percent change in DA diameter was comparable between hypoxic kits $(-2.2 \pm 9.3 \%, \mathrm{n}=5)$ and normoxic kits treated with $100 \mu \mathrm{M}$ S1QEL (-19.1 $\pm 15.1 \%, \mathrm{n}=5)$, indicating S1QEL mimicked hypoxia and preserved DA patency despite normoxic ventilation. These results suggest that suppression of site $\mathrm{I}_{\mathrm{Q}}$ electron leak via the actions of $\mathrm{S} 1 \mathrm{QEL}$ is capable of reversing ductal constriction in vivo. 
A)

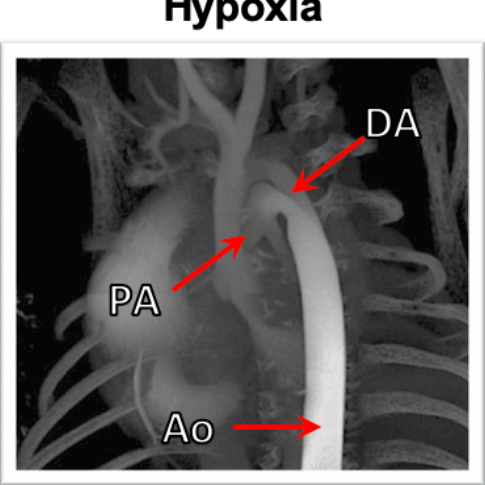

B)

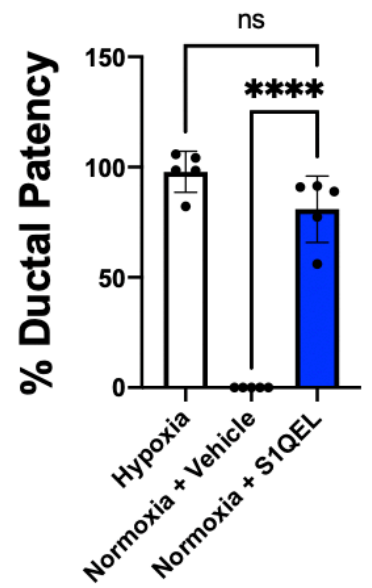

Normoxia

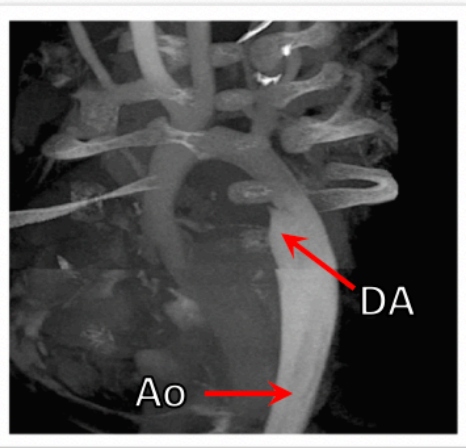

Normoxia + S1QEL

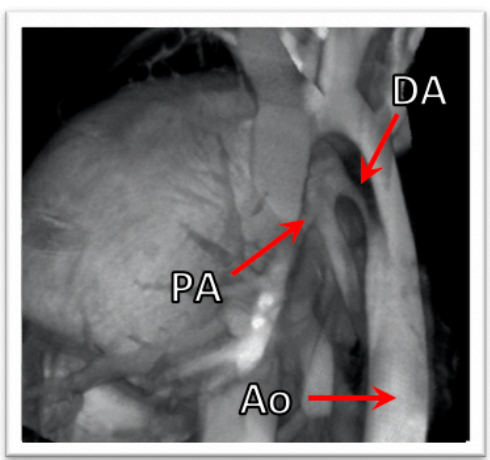

C)

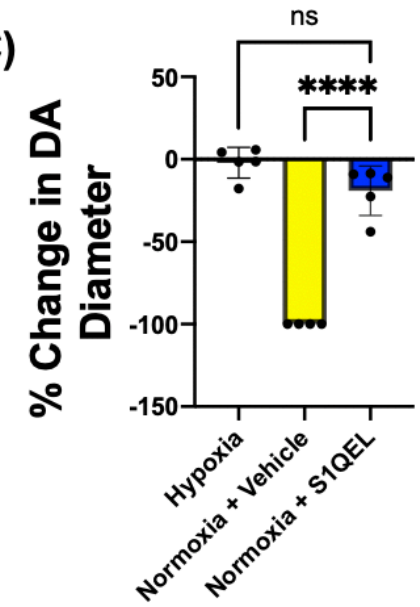

Figure 8 Effect of S1QEL on DA patency in vivo. In panel (A), maximum intensity projection (MIP) images were taken after perfusion with the contrast agent Omnipaque (with 3\% gelatin) and subsequent microCT imaging. On the left, the DA is shown connecting to the pulmonary arteries (PA) and descending thoracic aorta (Ao) in rabbit kits perfused immediately following cesarean delivery (hypoxia). In the middle, only the beak of the DA remains at its connection to the aorta, for rabbit kits exposed to 20 minutes of $100 \% \mathrm{O}_{2}$ (normoxia) prior to perfusion, whereas the connection between the DA and PA and Ao is observed for rabbit kits exposed to normoxia plus $100 \mu \mathrm{M}$ SIQEL prior to perfusion, as shown on the right. Percent DA patency (B) and change in DA diameter $(C)$ were quantified for rabbit kits exposed to hypoxia (white), normoxia with vehicle (DMSO, yellow), and normoxia with $100 \mu M$ S1QEL (blue) ( $n=5$ per group). **** $p<0.0001$. 


\section{Chapter 5}

\section{Discussion}

This study explored the role of electron leak suppressors S1QEL and S3QEL on oxygen sensing in the DA. This research has identified, for the first time, a critical role for electron leak from ETC complexes in ROS mediated oxygen signaling in DASMC, confirming previous studies showing ETC complex inhibitors were capable of modulating DA tone ex vivo by controlling ROS production. However, our findings extend prior work using classical ETC inhibitors like rotenone and show that electron leak and subsequent superoxide production by Complex I, but not Complex III, is critical for DASMC signaling in response to normoxia. Furthermore, our research indicates that electron leak suppressors and ETC inhibitors modulate DA tone through changes in ROS production, rather than by altering mitochondrial respiration or ATP production. This work also highlights the therapeutic potential for site $\mathrm{I}_{\mathrm{Q}}$ electron leak suppressors in modulating DA tone in vivo, which has potential translational relevance in the treatment of newborns with congenital heart abnormalities.

Using an organ bath system, we have demonstrated robust vasoconstriction and vasodilation responses of isolated rabbit DA vessels in response to changes in oxygen content (35-155 mmHg pO2). We have also demonstrated that the site $\mathrm{I}_{\mathrm{Q}}$ electron leak suppressor S1QEL, but not the site $\mathrm{III}_{\mathrm{Q}}$ electron leak suppressor S3QEL, dilates the normoxia induced preconstricted DA (Figure 2A-C). Importantly, this dilatory effect caused by S1QEL is not due to DA damage or a nonspecific suppression of tone, because phenylephrine constriction is unaltered in its presence (Figure 2D). This vasodilatory effect observed ex vivo suggests an importance for site $\mathrm{I}_{\mathrm{Q}}$ in oxygen sensing in the DA. Our research is also in agreement with our prior studies 
showing that ETC complex inhibitors rotenone and antimycin A cause dilation of the DA ex vivo (Figure 2C) [8].

Oxygen sensing by the DA, like other tissues of the HOSS, is known to be mediated by changes in ROS production by the mitochondria. These ROS diffuse from mitochondria outwards to the peripheral parts of the cell to modulate redox sensitive ion channels, which can cause calcium influx following membrane depolarization. In DASMC, it has been shown that normoxic vasoconstriction is triggered by increased ROS production, which is required for normoxia induced $\mathrm{K}^{+}$channel inhibition [71]. Our research further validates this proposed cell signaling mechanism, and shows that normoxia causes increased superoxide production in DASMC (Figure 6A/B). Site $\mathrm{I}_{\mathrm{Q}}$, or Complex I quinone binding site, is known to be a major source of ROS production in various tissue types $[12,85,86]$. Taken together with the data we obtained measuring ROS production in hDASMC (Figure 6 and 7), these findings suggest that site $\mathrm{I}_{\mathrm{Q}}$, but not site $\mathrm{III}_{\mathrm{Q}}$, may be a significant source of superoxide production required for cell signaling during oxygen sensing in DASMC.

Our ex vivo tissue organ bath work and in vitro ROS measurements highlight important similarities between the Complex I inhibitor rotenone and electron leak suppressor S1QEL. Both of these compounds are shown to cause vasodilation of the DA ex vivo, as well as decrease normoxic induced rises in ROS production. Our in vitro work using the Seahorse XF Mito Stress Test has shown that S1QEL and rotenone have different effects on mitochondrial respiratory parameters. The OCR tracings observed in DASMC exposed to S1QEL and S3QEL show similar trends in OCR values over the course of oligomycin, FCCP, and antimycin A/rotenone injections (Figure 4A/B). In contrast to these electron leak suppressors, rotenone completely abolishes mitochondrial-linked OCR immediately upon injection, and the OCR in rotenone-treated cells 
remain low over the remainder of these experiments (Figure 4A). Quantitative analysis of these OCR tracings across rbDASMC lines has shown that S1QEL, but not rotenone, maintains key parameters linked to mitochondrial respiration, including spare respiratory capacity and ATP production (Figure 5D/F). Taken together with our findings from the organ bath experiments showing greater reductions in DA tension after treatment with S1QEL compared to rotenone, this data suggests that although changes in parameters linked to mitochondrial metabolism are associated with DASMC oxygen sensing, they are not the driving force behind these signaling mechanisms; rather it is ROS related to ETC leak that is the signaling mechanism.

Our research investigating the impact of S1QEL on DA tone in vivo suggests that electron leak suppressors have the potential to act as novel therapeutics for controlling DA tone. Unlike rotenone which is known to be toxic and is even used to create animal models of Parkinson's disease [87], S1QEL has been shown to be non-toxic across a variety of cell types and has already been used to improve survival rates in mice models of asystolic cardiac arrest $[12,88]$. Our work demonstrates that S1QEL is capable of reversing oxygen induced constriction of the DA in rabbit term kits (Figure 8). This has potential translational relevance for newborns with congenital heart disease, such as those with single ventricles, who depend on PDA for maintaining their systemic or pulmonary circulation, such as in newborns afflicted with pulmonary atresia or aortic stenosis [89].

Our research is in agreement with the redox theory of oxygen sensing, which postulates that ROS production by the ETC is proportionate to rates of mitochondrial respiration [90]. This implies that when rates of respiration are low due to low oxygen supply during hypoxia, rates of electron flux through the ETC remain low, causing little electron leak and ROS production. Similarly, as rates of respiration increase with increased oxygen supply during normoxia, 
increased electron flux through the ETC increases electron leak and subsequent ROS production by the ETC. As it relates to oxygen sensing in the DA, site IQ may constitute a key electron leak site which when bound by S1QEL may prevent adequate ROS production by the mitochondria to sustain ROS mediated DA constriction. Interestingly, site $\mathrm{I}_{\mathrm{Q}}$ is the same site bound by the Complex I inhibitor rotenone, further highlighting its importance along with Complex I in oxygen sensing in the DA. Although S3QEL was not observed to elicit the same vasodilatory effects, antimycin A, which also binds Complex III is known to cause dilation of the preconstricted DA, as was observed in our organ bath experiments (Figure 2C). Interestingly, S3QEL binds to site $\mathrm{Q}_{0}$, or the quinone oxidation site of Complex III, whereas antimycin A binds to site $\mathrm{Q}_{\mathrm{i}}$, or the quinone reductase site (Figure 14). Although $\mathrm{Q}_{\mathrm{o}}$ is known to be a source of ROS production in diverse cell signaling events and pathologies [91], our data suggests that ROS production by this site is not implicated in oxygen sensing in the DA. Site $\mathrm{Q}_{\mathrm{i}}$ of Complex III is not believed to be a major source of electron leak and ROS production by the ETC, suggesting that the vasodilatory effects of antimycin A may be caused by a general decrease in electron leak from the ETC as a result of decreased overall electron flux due to Complex III inhibition.

The significance of site $\mathrm{I}_{\mathrm{Q}}$ in oxygen sensing has recently been demonstrated to be important in the HPV response of PASMC. In response to hypoxia, ROS production by the mitochondria of PASMC is decreased, leading to inhibition of redox-sensitive $\mathrm{Kv}$ channels. Closure of these channels results in PASMC depolarization and vasoconstriction, following an influx of $\mathrm{Ca}^{2+}$ through voltage-sensitive $\mathrm{Ca}^{2+}$ channels. Dunham-Snary et al. found that Ndufs2, a subunit of Complex I found at the $\mathrm{I}_{\mathrm{Q}}$ site, is required for oxygen-sensing and HPV in PASMC [77]. Treatment with siNdufs2 prevented hypoxic increases in $\left[\mathrm{Ca}^{2+}\right]$ in PASMC, and significantly impaired hypoxia induced constriction in vivo in rats. Ndufs 2 is located at the distal 
portion of the iron-sulfur (Fe-S) chain that runs through Complex I, and forms part of the binding pocket of the terminal Fe-S cluster, N2, which transfer electrons to ubiquinone at site $\mathrm{I}_{\mathrm{Q}}$ (Figure 13). Taken together with our research, site $I_{Q}$ may represent a significant site for ROS production and cell signaling events across a variety of tissues included in the HOSS. These findings suggest potential areas for future research looking at the role of Ndufs2 in oxygen sensing within the DASMC, as well as the impact of S1QEL and other site IQ electron leak suppressors on oxygen sensing within the pulmonary vasculature. 


\section{Chapter 6}

\section{Conclusions}

This work demonstrates the importance of electron leak and ROS production from Complex I in DA oxygen sensing. We have also identified S1QEL as a potential novel therapeutic for vasodilating the DA both ex vivo and in vivo, and have demonstrated its ability to modulate ROS production without inhibiting key mitochondrial respiratory parameters in vitro. This work suggests that Complex I, but not Complex III, is of significant importance in initiating ROS mediated cell signaling mechanisms required for DASMC oxygen-sensing, and further highlights the importance of site IQ-generated ROS which have been observed to play a role in other specialized oxygen sensing tissues. 


\title{
Chapter 7
}

\section{Mitochondrial Iron-Sulfur Clusters: Structure, Function, and an Emerging Role in Vascular Biology}

\author{
Austin D Read ${ }^{1, \#}$, Rachel ET Bentley ${ }^{1, \#}$, Stephen L Archer ${ }^{1,2}$, Kimberly J Dunham-Snary ${ }^{1,3, *}$ \\ 1 - Department of Medicine, Queen's University, Kingston, ON, CAN \\ 2 - Queen's CardioPulmonary Unit, Queen's University, Kingston, ON, CAN \\ 3 - Department of Biomedical and Molecular Sciences, Queen's University, Kingston, ON, CAN \\ \# - equal contributor \\ * - corresponding author
}

This review article was published in Redox Biology (DOI: 10.1016/j.redox.2021.102164), and has been included in my thesis in accordance with Elsevier's copyright rules.

\subsection{Abstract}

Iron-sulfur (Fe-S) clusters are essential cofactors most commonly known for their role mediating electron transfer within the mitochondrial respiratory chain. The Fe-S cluster pathways that function within the respiratory complexes are highly conserved between bacteria and the mitochondria of eukaryotic cells. Within the electron transport chain, Fe-S clusters play a critical role in transporting electrons through Complexes I, II and III to cytochrome c, before subsequent transfer to molecular oxygen. Fe-S clusters are also among the binding sites of classical mitochondrial inhibitors, such as rotenone, and play an important role in the production of mitochondrial reactive oxygen species (ROS). Mitochondrial Fe-S clusters also play a critical role in the pathogenesis of disease. High levels of ROS produced at these sites can cause cell injury or death, however, when produced at low levels can serve as signaling molecules. For example, Ndufs2, a Complex I subunit containing an Fe-S center, N2, has recently been identified as a redox- 
sensitive oxygen sensor, mediating homeostatic oxygen-sensing in the pulmonary vasculature and carotid body. Fe-S clusters are emerging as transcriptionally-regulated mediators in disease and play a crucial role in normal physiology, offering potential new therapeutic targets for diseases including malaria, diabetes, and cancer.

\subsection{Introduction}

Iron-sulfur (Fe-S) clusters are among the most common cofactors observed across nature and comprise the largest class of metalloproteins. These clusters are structurally diverse, existing in simple forms such as $[1 \mathrm{Fe}-0 \mathrm{~S}]$, where a single iron atom is coordinated by four cysteine groups, found in rubredoxins of sulfur-metabolizing archaea, and in complex forms such as the [8Fe-7S] found in nitrogenases of nitrogen-fixing bacteria [92, 93]. Most commonly, these clusters exist as [2Fe-2S], [3Fe-4S] or [4Fe-4S]. The simplest of these, [2Fe-2S] is a rhombic structure containing two iron atoms bridged by two sulfur atoms, and is the cluster commonly found within ferredoxins, such as adrenodoxin, an Fe-S protein involved in the synthesis of steroids within the adrenal glands (Figure 9) [94]. Although typically ligated by four cysteine residues within the Fe-S protein, ligation involving both cysteine and histidine residues is also observed, as seen in the Rieske iron-sulfur protein within Complex III of the electron transport chain (ETC) [95]. The [4Fe-4S] cluster exists in a cubic structure, with iron and sulfur atoms found in alternating corner positions. These clusters are found in bacterial ferredoxins, and within the mitochondrial respiratory complexes, such as with cluster N2 located within Complex I of the ETC [96]. The [3Fe-4S] clusters, although less common then the [4Fe-4S] subtype, are also ubiquitous, existing in a variety of ferredoxins and in the quinone binding site of Complex II [97]. 


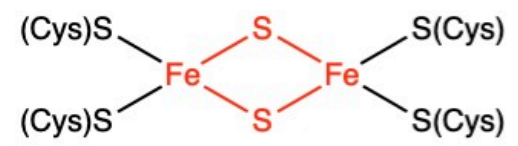

[2Fe-2S]

Adrenodoxin

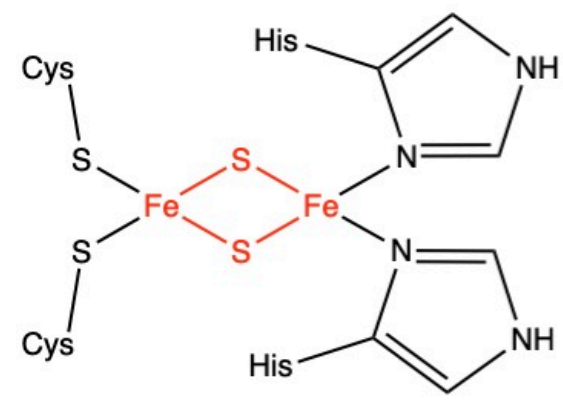

$[2 \mathrm{Fe}-2 \mathrm{~S}]$

Complex III Rieske

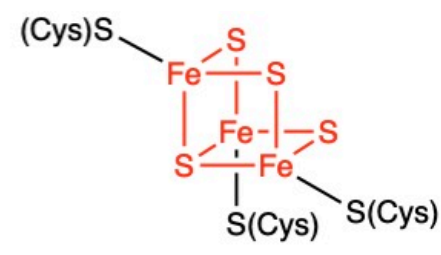

$[3 \mathrm{Fe}-4 \mathrm{~S}]$

Complex II

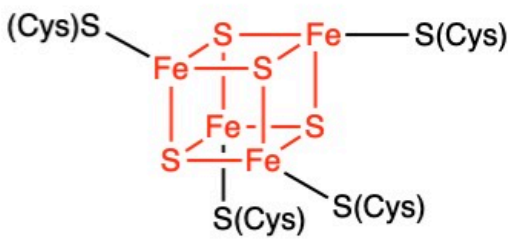

[4Fe-4S]

Complex I N2

Figure 9 The most common forms of Fe-S clusters found in biological systems: [2Fe-2S], [3Fe-4S] and [4Fe-4S]. In all structures shown above, the iron and sulfur atoms that are a part of the cluster are shown in red and ligating amino acid residues are shown in black.

Fe-S clusters are versatile protein prosthetic groups and serve a variety of functions in biological systems. They function as cofactors in enzyme catalysis, and are often situated in active sites to assist in Lewis acid reactions, as observed with mitochondrial aconitase and radical S-adenosylmethionine (SAM) enzymes $[98,99]$. These clusters also have regulatory roles, modulating gene expression in response to oxidative stress (via superoxide response (SoxR) proteins), oxygen levels (via fumarate-nitrate reduction (FNR) proteins), as well as iron levels (via Iron Response Proteins including IRP1 and IRP2) [100-102]. Fe-S clusters have also been observed to play important roles in DNA metabolism, coordinating protein conformational changes during DNA replication and repair, as found in primases, helicases, nucleases, and polymerases [103-107]. Within these enzymes, these clusters are involved in maintaining structural stability, and are also known to play roles in DNA binding, unwinding, and exonuclease activity. For example, an Fe-S cluster within the helicase-nuclease AddAB is found 
adjacent to the enzyme's wedge domain and is essential for DNA unwinding, although its exact function is unknown [108].

Despite these diverse roles, Fe-S clusters are most commonly known for their role in electron transfer, with their arrangements defining the pathways of electron transport within the systems that drive photosynthesis and mitochondrial respiration. Within the mitochondria, Fe-S centers play vital roles in both the tricarboxylic acid cycle (TCA) and the ETC. Electrons donated by both $\mathrm{NADH}$ and $\mathrm{FADH}_{2}$ are transferred through numerous Fe-S clusters found in complexes I, II and III of the ETC, with molecular oxygen serving as the terminal electron acceptor, forming water. In this review, we describe the evolution of Fe-S clusters across taxa, their roles in mitochondrial respiration, with an emphasis on their composition within Complex I of the ETC, their emerging roles as oxygen sensors within the pulmonary arteries, the carotid body, and the ductus arteriosus, as well as emerging targets of therapeutics.

\subsection{Mitochondria and endosymbiosis}

It is important to explore the origins of mitochondria to provide context for the roles of mitochondrial Fe-S clusters. The structure of the mitochondrion was first described in 1888, with Kölliker concluding that all mitochondria, then considered "granules", have a membrane [109, 110]. The origins of mitochondria as bacterial endosymbionts were first proposed by Wallin in 1927 [111]. However, the endosymbiont hypothesis, outlined in Figure 10, was popularized by Lynn Margulis (then Lynn Sagan) in her 1967 publication [112], and in more detail in her subsequent books $[113,114]$. Margulis synthesized ideas from a variety of sources to create a clear theory of endosymbiosis $[111,115-118]$. There was previously a great deal of debate between the opposing hypotheses of endosymbiosis and autogenous origins of mitochondria [119-125], with the autogenous origin theory postulating that mitochondria arose from within a 
single cell via functional specialization and intracellular compartmentalization. However, endosymbiosis is now universally accepted as the evolutionary origin of mitochondria; that a proto-eukaryote (heterotrophic anaerobe) engulfed a prokaryotic proto-mitochondrion [112]. Genetic analyses have determined that mitochondria are likely descended from -proteobacteria $[126,127]$, with the closest relative of the proto-mitochondrion belonging to the order Rickettsiales $[128,129]$.

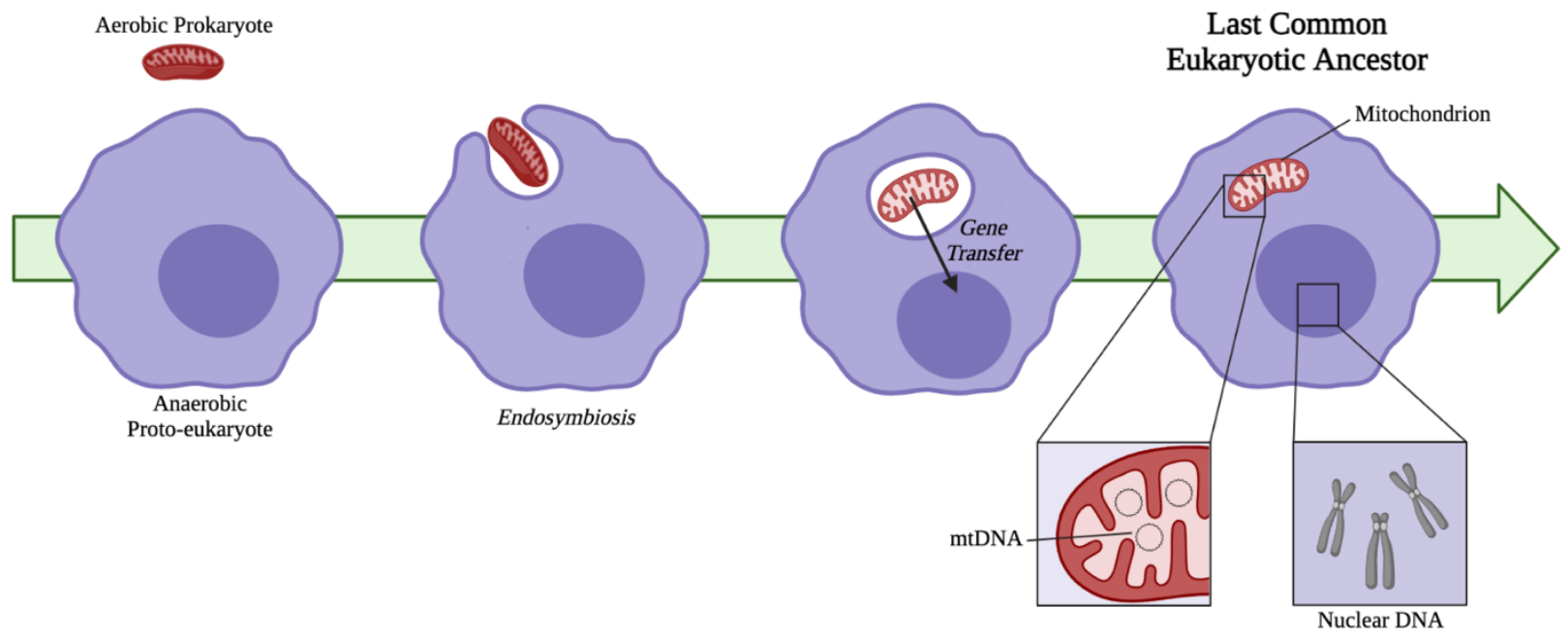

Figure 10 Overview of endosymbiont hypothesis. This universally accepted theory of mitochondrial origins posits that the anaerobic proto-eukaryote engulfed the aerobic prokaryote, most closely related to the order Rickettsiales. There was sizeable transfer of genes encoding mitochondrial proteins to the nuclear genome and elimination of redundancy, with current mitochondrial genomes encoding as few as 5 genes. The acquisition of the mitochondrion resulted in the last common eukaryotic ancestor, from which all current eukaryotes evolved.

Debate continues surrounding the initial nature of the relationship of the protomitochondrion and proto-eukaryote, and about the selective advantage provided by the protomitochondrion immediately following endosymbiosis $[130,131]$. Some suggest the first endosymbiont was an aerobic heterotroph that provided an advantage to the host by supporting its energy need via secretion of ATP [113], removing the fermentation waste of the host [132, 133], and/or removing intracellular oxygen that could be damaging or toxic to the host [134]. There is also evidence indicating that the benefit provided by the first mitochondrial endosymbiont was the generation of heat, enabling the host to colonize cooler environments with 
a higher internal temperature maintaining membrane fluidity, DNA helical tension, enzymatic reaction rates and other temperature-dependent cellular processes [131]. It has been postulated that assembly of Fe-S clusters was the essential contribution of the endosymbiont and the reason for the existence of the mitochondrion $[135,136]$, as Fe-S cluster synthesis is essential for eukaryotic cell survival [137-139]. Others have proposed a non-mutualistic scenario, where the proto-mitochondrion was a predator invading the host $[140,141]$. These theories are based on current knowledge and understanding of $\quad$-proteobacterial diversity [130]. While these theories are not mutually exclusive, no consensus has been reached as to the precise nature of the initial host-symbiont relationship.

While a primary function of the organelle remains the generation of ATP via the respiratory chain, mitochondria exhibit significant diversity between cells and tissues and display tissue heterogeneity in their noncanonical functions (beyond generation of ATP), including energy production, calcium homeostasis, and coordination of programmed cell death (apoptosis). The mammalian respiratory chain is composed of five complexes, each with catalytic subunits highly conserved from bacteria [142-146]. These subunits function to couple electron transport between subunits to proton translocation across the inner mitochondrial membrane (IMM), creating the proton motive force required for ATP synthesis. While mitochondrial subunits are highly conserved, there still exists a great deal of mitochondrial and respiratory chain diversity within the eukaryotic domain. While all eukaryotes are descended from a common ancestor, some now lack subunits of the respiratory chain, and others, such as Giardia muris [147] and Entamoeba histolytica [148], lack mitochondria altogether, instead relying on hydrogenosomes for the anaerobic generation of ATP [149]. 
The fungal respiratory chain is similar to that of other eukaryotes, with a few notable differences. Complex I has been found in nearly all species of fungi, with Complex I having a similar structure to its counterpart in other forms of life. Notably, Saccharomyces cerevisiae lacks Complex I, as do certain other fungi [150]; these organisms possess alternative NADH:ubiquinone oxidoreductases that enable direct oxidation of external NADH or act as a parallel, rotenone-insensitive route of internal NADH oxidation [151]. Alternative NADH:ubiquinone oxidoreductases are widely distributed in fungi and plants, and lack Fe-S clusters [152]. These alternative NADH dehydrogenases are nuclear-encoded and, unlike traditional Complex I, do not couple electron transport to proton translocation across the IMM $[153,154]$. These alternative pathways in plants and fungi can function separate from or in conjunction with the canonical pathway [151].

In addition to alternative forms of Complex I, 'Alternative Oxidase' is present in most fungal genomes tested [155] and acts in parallel to Complex III to oxidize ubiquinol [156]. Alternative Oxidase also catalyzes the reduction of molecular oxygen to water [156]. Alternative Oxidase does not contain any Fe-S clusters and is not susceptible to the Complex III inhibitors antimycin A and myxothiazol, nor to the Complex IV inhibitor cyanide [155-157]. In certain plant species, alternative oxidase plays a vital role for heat production, whether to volatilize insect attractants, as is the case in the Arum plant, or to permit growth at lower temperatures, as seen in the American and Asian skunk cabbage [158]. However, it is generally considered to be a stress protein induced when factors such as growth inhibition or exposure to wounding, drought, or adverse salinity impair the main respiratory chain [158].

\subsection{Mitochondrial Fe-S clusters}

\subsubsection{Aconitase Fe-S clusters}


Aconitases are metalloenzymes containing a [4Fe-4S] cluster, acting in both the cytosol and mitochondria in different capacities. In the cytosol, the iron response protein (IRP-1) acts as a sensor of iron levels within the cell. When iron concentrations are high, IRP-1 acts as an aconitase and contains a [4Fe-4S] Fe-S cluster. When iron levels are low, IRP-1 loses its Fe-S cluster and the apoprotein subsequently binds to iron-responsive elements (IREs) within the 5' or 3' UTR of target mRNA to promote iron uptake, and reduce iron storage and utilization [159, 160]. Within the mitochondria, aconitase acts in the second step of the TCA cycle, converting citrate to isocitrate via the intermediate cis-aconitate. The enzyme exists in two forms: an inactive form containing a [3Fe-4S] cluster, and upon acquiring another iron atom, an active form containing a $[4 \mathrm{Fe}-4 \mathrm{~S}]$ cluster. This additional labile iron atom $\left(\mathrm{Fe}_{\alpha}\right)$ is coordinated by water molecules and is essential for carrying out the enzyme's catalytic function (Figure 11) [161]. The $\mathrm{Fe}_{\alpha}$ atom within active aconitase coordinates with oxygen atoms of citrate and water and acts as a Lewis acid to activate the hydroxyl group of citrate to facilitate the isomerization reaction.
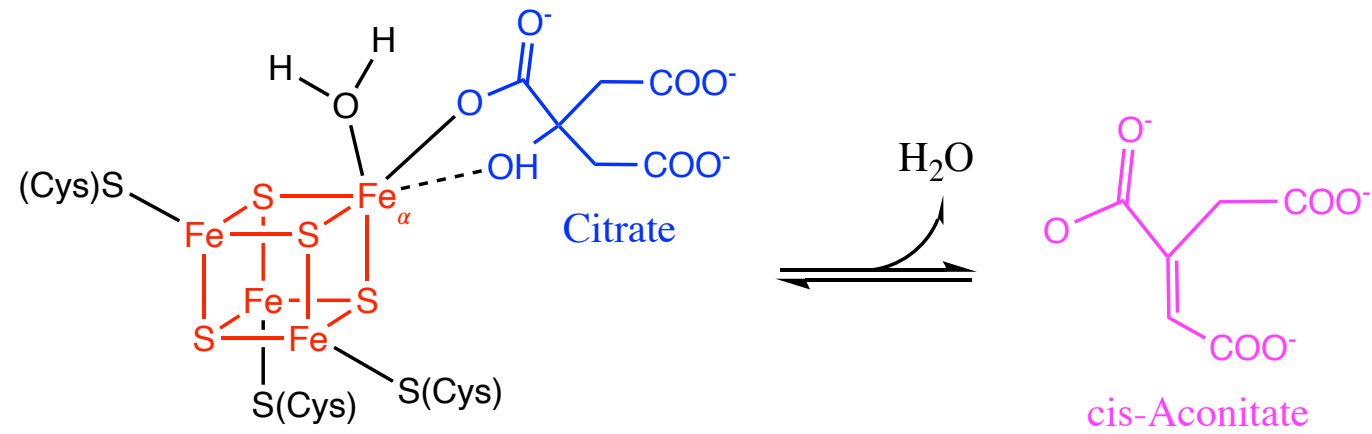

Figure 11 Aconitase reaction mechanism. The isomerization reaction of citrate (shown in blue) to cis-Aconitate (magenta) is facilitated by the [4Fe-4S] cluster found within aconitase (shown in red). The addition of another labile iron (Fe $\left.{ }_{\alpha}\right)$ transforms inactive aconitase into its active form, and coordinates with water molecules and oxygen atoms of citrate to facilitate enzyme catalysis.

Aconitase, as well as other Fe-S cluster-containing dehydratases, are sensitive to both reactive oxygen species (ROS), including superoxide $\left(\mathrm{O}_{2} \cdot \cdot^{-}\right)$and hydrogen peroxide $\left(\mathrm{H}_{2} \mathrm{O}_{2}\right)$, and 
the production of the reactive nitrogen species (RNS) peroxynitrite (ONOO-) [162-164]. The ETC is a major source of both ROS and RNS within the mitochondria. When levels of ROS/RNS rise, these molecules are capable of oxidizing the Fe-S cluster in active m-aconitase, causing the loss of the labile $\mathrm{Fe}_{\alpha}$ atom and enzyme inactivation [165]. This susceptibility of m-aconitase to ROS/RNS-induced inactivation makes it a candidate sensor for redox changes within the mitochondria. If levels of oxidative stress increase, aconitase inactivation slows the flow of metabolites through the TCA cycle, thereby decreasing the availability of substrates for the ETC, and possibly promoting fatty acid synthesis through the export of citrate out of the mitochondrion [165]. In this way, the sensitivity of Fe-S cluster inactivation in m-aconitase may allow the enzyme to act as a sensor of ROS/RNS production by controlling metabolic flux through the mitochondria.

\subsubsection{Electron transport chain Fe-S clusters}

Oxidative phosphorylation describes a series of metabolic reactions that take place within the mitochondria, responsible for the majority of ATP production within aerobic eukaryotes. The electron carriers $\mathrm{NADH}$ and $\mathrm{FADH}_{2}$ formed from glycolysis, fatty acid oxidation, and the TCA cycle act as electron donors, passing electrons through four respiratory complexes of the ETC, to eventually reduce molecular oxygen to water. As electrons pass through the ETC complexes, protons are pumped from the mitochondrial matrix into the intermembrane space (IMS), creating a transmembrane electrical potential that is used to drive ATP production by ATP synthase (Complex V). Various redox groups exist within the respiratory complexes to facilitate the movement of electrons from $\mathrm{NADH}$ and $\mathrm{FADH}_{2}$ through the ETC, including flavins, quinones, hemes, and Fe-S clusters (Figure 12). Within the mitochondria, Fe-S clusters play a variety of roles, acting not only in the main electron transfer pathways required for ATP generation, but 
also acting in mitochondrial DNA (mtDNA) catabolism. Within the pathways which drive mitochondrial respiration, Fe-S clusters are found within three of the four complexes which make up the ETC. In coordination with other redox groups, Fe-S clusters within Complexes I and II form electron tunneling chains, transferring electrons one at a time from the electron carriers $\mathrm{FADH}_{2}$ and $\mathrm{NADH}$ to ubiquinone. Although not found in a chain, an additional Fe-S cluster found within Complex III plays an integral role in passing electrons from ubiquinol (the reduced version of ubiquinone) to cytochrome $\mathrm{c}$, to facilitate further transfer of electrons to Complex IV. In the mitochondria, Fe-S biogenesis involves the coordination of several proteins, including iron-sulfur cluster scaffold (ISCU) which provides the cysteine ligands where upon new Fe-S clusters are synthesized, cysteine desulfurase (NFS1) which provides the inorganic sulfur to ISCU, and frataxin $(\mathrm{FXN})$ a protein that regulates desulfurase activity and has been suggested to act as an iron chaperone, delivering iron to the scaffold. After synthesis of [2Fe-2S] clusters in the mitochondria, they are either transferred to apoproteins, exported into the cytosol for incorporation into the cytosolic iron-sulfur cluster assembly (CIA) pathway, or further processed into [4Fe-4S] clusters. Although not the subject of this review, biogenesis of Fe-S clusters has been extensively studied and recently review by Alfadhel et al. and Maio et al. [166, $167]$. 


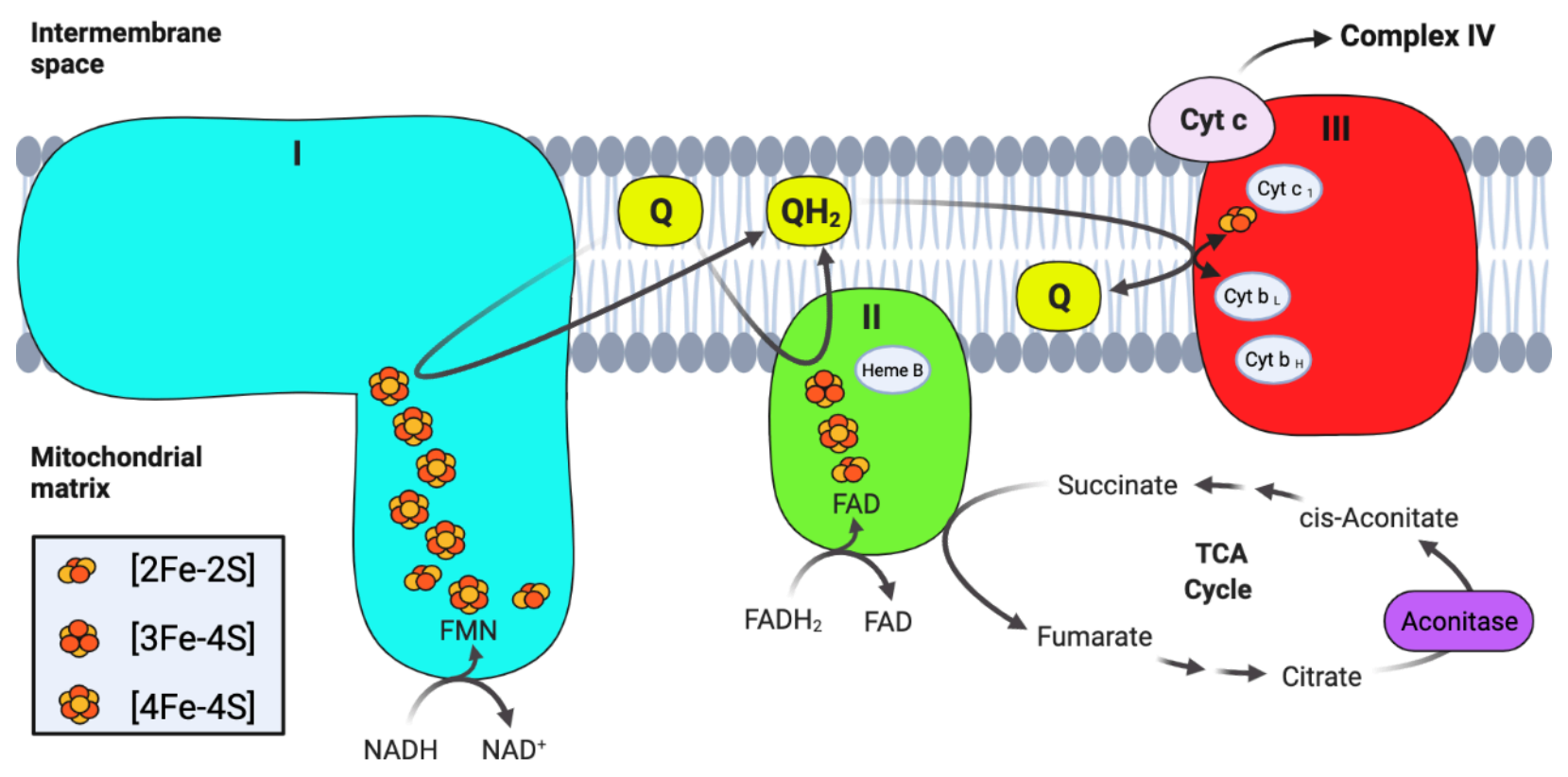

Figure 12 Simplified version of the mitochondrial ETC, showing complexes I (blue), II (green), and III (red). The electron donors $\mathrm{NADH}$ and $\mathrm{FADH}_{2}$ are shown reducing complexes I and II, respectively, as well as the electron carriers' ubiquinone (Q) and ubiquinol $\left(\mathrm{QH}_{2}\right)$. Besides the Fe-S clusters, other redox prosthetic groups within each complex are also shown, including FMN and FAD, as well as the heme moieties a part of the cytochrome proteins. Intermediates within the TCA cycle that interact with Complex II and aconitase (purple) are also shown.

\subsubsection{Fe-S clusters in Complex I}

Complex I, also referred to as NADH dehydrogenase, is L-shaped, containing a membrane portion connected to a hydrophilic peripheral portion which protrudes into the mitochondrial matrix (or cytoplasm in bacteria) [168-170]. Although a total of 45 different subunits comprise the mammalian Complex I, a core of 14 subunits is sufficient to drive energy production in the prokaryotic version of the complex [171-173]. These core 14 subunits are highly conserved across both prokaryotes and eukaryotes, and are considered to be the minimal assembly of Complex I [174]. In the mammalian complex, these subunits can be split into two groups, hydrophobic subunits encoded by the mitochondrial genome and hydrophilic subunits encoded by the nuclear genome. These subunits are further subdivided into the three functional modules of Complex I: the electron input/dehydrogenase $(\mathrm{N})$ module that accepts electron via the oxidation of $\mathrm{NADH}$, the electron output/hydrogenase $(\mathrm{Q})$ module that reduces ubiquinone, 
and the proton translocation $(\mathrm{P})$ module that pumps protons into the IMS [175]. Table 1

summarizes the 14 core subunits found across three species: Homo sapiens, Escherichia coli and Neurospora crassa. The location of the genes encoding each subunit in the human nuclear and mitochondrial genomes, the module where each subunit is found, as well as the presence of any ligated Fe-S clusters are also listed. Note that in E. coli, as with a few other bacteria, two of the core 14 subunits are fused together (nuoC and nuoD), and the complex therefore contains 13 subunits total [176].

\begin{tabular}{|c|c|c|c|c|c|}
\hline Species & $\begin{array}{l}\text { Subunit } \\
\text { Name }\end{array}$ & $\begin{array}{l}\% \text { Homology Relative } \\
\text { to H. Sapiens }\end{array}$ & $\begin{array}{l}\text { Gene Location } \\
\text { Homo Sapiens } \\
\end{array}$ & Module & $\begin{array}{l}\text { Associated } \\
\text { Fe-S Clusters } \\
\end{array}$ \\
\hline H. sapiens & \multirow{3}{*}{$\begin{array}{l}\text { NDUFV1 } \\
\text { nuoF } \\
51 \mathrm{kDa} \\
\end{array}$} & \multirow{3}{*}{$\begin{array}{l}- \\
62 * \\
86^{*} \\
\end{array}$} & \multirow[t]{3}{*}{$11 \mathrm{q} 13$} & \multirow[t]{3}{*}{$\mathrm{N}$} & \multirow[t]{3}{*}{$\mathrm{N} 3[4 \mathrm{Fe}-4 \mathrm{~S}]$} \\
\hline E. coli & & & & & \\
\hline N. crassa & & & & & \\
\hline H. sapiens & \multirow{3}{*}{$\begin{array}{l}\text { NDUFV2 } \\
\text { nuoE } \\
24 \mathrm{kDa} \\
\end{array}$} & \multirow{3}{*}{$\begin{array}{l}- \\
57 * \\
77 *\end{array}$} & \multirow[t]{3}{*}{ 18p11.31-p11.2 } & \multirow[t]{3}{*}{$\mathrm{N}$} & \multirow[t]{3}{*}{$\mathrm{N} 1 \mathrm{a}[2 \mathrm{Fe}-2 \mathrm{~S}$} \\
\hline E. coli & & & & & \\
\hline N. crassa & & & & & \\
\hline H. sapiens & \multirow{3}{*}{$\begin{array}{l}\text { NDUFS1 } \\
\text { nuoG } \\
78 \mathrm{kDa}\end{array}$} & \multirow{3}{*}{$\begin{array}{l}- \\
42 * \\
69 *\end{array}$} & \multirow[t]{3}{*}{$2 q 33-q 34$} & \multirow[t]{3}{*}{$\mathrm{N}$} & $\mathrm{N} 1 \mathrm{~b}[2 \mathrm{Fe}-2 \mathrm{~S}]$ \\
\hline E. coli & & & & & $\mathrm{N} 4[4 \mathrm{Fe}-4 \mathrm{~S}]$ \\
\hline N. crassa & & & & & N5 [4Fe-4S] \\
\hline H. sapiens & \multirow{3}{*}{$\begin{array}{l}\text { NDUFS2 } \\
\text { nuoD } \\
49 \mathrm{kDa}\end{array}$} & \multirow{3}{*}{$\begin{array}{l}- \\
60 * \\
85 *\end{array}$} & \multirow[t]{3}{*}{$1 \mathrm{q} 23$} & \multirow[t]{3}{*}{ Q } & \\
\hline E. coli & & & & & \\
\hline N. crassa & & & & & \\
\hline H. sapiens & \multirow{3}{*}{$\begin{array}{l}\text { NDUFS3 } \\
\text { nuoC } \\
30.4 \mathrm{kDa}\end{array}$} & \multirow{3}{*}{$\begin{array}{l}- \\
60 * \\
68\end{array}$} & \multirow[t]{3}{*}{$11 \mathrm{p} 11.11$} & \multirow[t]{3}{*}{ Q } & \\
\hline E. coli & & & & & \\
\hline N. crassa & & & & & \\
\hline H. sapiens & \multirow{3}{*}{$\begin{array}{l}\text { NDUFS7 } \\
\text { nuoB } \\
19.3 \mathrm{kDa}\end{array}$} & \multirow{3}{*}{$\begin{array}{l}- \\
76 * \\
77^{*}\end{array}$} & \multirow[t]{3}{*}{$19 \mathrm{p} 13.3$} & \multirow[t]{3}{*}{ Q } & \multirow[t]{3}{*}{$\mathrm{N} 2[4 \mathrm{Fe}-4 \mathrm{~S}]$} \\
\hline E. coli & & & & & \\
\hline N. crassa & & & & & \\
\hline H. sapiens & \multirow{3}{*}{$\begin{array}{l}\text { NDUFS8 } \\
\text { nuoI } \\
21.3 \mathrm{c}\end{array}$} & \multirow{3}{*}{$\begin{array}{l}- \\
55 \\
84\end{array}$} & \multirow[t]{3}{*}{$11 \mathrm{q} 13$} & Q & $\mathrm{N} 6 \mathrm{a}[4 \mathrm{Fe}-4 \mathrm{~S}]$ \\
\hline E. coli & & & & & $\mathrm{N} 6 \mathrm{~b}[4 \mathrm{Fe}-4 \mathrm{~S}]$ \\
\hline N. crassa & & & & & \\
\hline H. sapiens & ND1 & - & mt_3307 & $\mathrm{P}$ & \\
\hline E. coli & nuoH & 61 & & & \\
\hline N. crassa & ND1 & 57 & & & \\
\hline H. sapiens & ND2 & - & mt_4470 & $\mathrm{P}$ & \\
\hline E. coli & nuoN & 45 & & & \\
\hline N. crassa & ND2 & 43 & & & \\
\hline H. sapiens & ND3 & - & mt_10059 & $\mathrm{P}$ & \\
\hline E. coli & nuoA & 53 & & & \\
\hline N. crassa & ND3 & 50 & & & \\
\hline H. sapiens & ND4 & - & mt_10760 & $\mathrm{P}$ & \\
\hline E. coli & nuoM & 52 & & & \\
\hline
\end{tabular}




\begin{tabular}{|c|c|c|c|c|}
\hline N. crassa & ND4 & 54 & & \\
\hline H. sapiens & ND4L & - & mt_10470 & $P$ \\
\hline E. coli & nuoK & 51 & & \\
\hline N. crassa & ND4L & 58 & & \\
\hline H. sapiens & ND5 & - & mt_12337 & $\mathrm{P}$ \\
\hline E. coli & nuoL & 54 & & \\
\hline$N$. crassa & ND5 & 64 & & \\
\hline H. sapiens & ND6 & - & mt_14149 & $P$ \\
\hline E. coli & nuoJ & & & \\
\hline N. crassa & ND6 & $\begin{array}{l}\text { No significant } \\
\text { similarity }\end{array}$ & & \\
\hline
\end{tabular}

Table 1. Complex I minimal assembly. Subunit names for H. sapiens, E. coli, and N. crassa shown. The location of the genes encoding H. sapiens subunits in the nuclear and mitochondrial genomes are shown in blue and red, respectively. Note that clusters N1 a and N5 have not been identified in N. crassa. [176-182] Percent homology values are reported as 'positives' (i.e. amino acids that are identical or have similar chemical properties); where multiple (H. sapiens) subunit isoforms exist, percent homology has been averaged across isoforms and indicated with an asterisk.

Of the 12 Fe-S clusters found within the ETC complexes, Complex I contains the majority, carrying eight clusters within five of its 14 core subunits. The Fe-S clusters found within Complex I are all located within the peripheral arm, in both the $\mathrm{N}$ and Q modules. The cluster chain begins at the NADH binding site near the flavin mononucleotide (FMN), and terminates at the ubiquinone binding site, at the interface of the peripheral and membrane arms. Figure 13 shows the Fe-S cluster chain within Complex I from B. taurus, with the midpoint redox potentials (a measure of the tendency of a chemical species to gain/lose electrons, with more positive values corresponding to a higher affinity for electrons) at $\mathrm{pH} 7\left(\mathrm{E}_{\mathrm{m}}\right)$ also shown in parentheses. Of these eight clusters, N1a and N1b are of the [2Fe-2S] form, while the remaining $\operatorname{six}(\mathrm{N} 2, \mathrm{~N} 3, \mathrm{~N} 4, \mathrm{~N} 5, \mathrm{~N} 6 \mathrm{a}$ and N6b) are of the [4Fe-4S] type. Note that the "N" prefix refers to the Fe-S clusters found within NADH dehydrogenase, whereas the numbering of each cluster corresponds to their spin relaxation rates measured through electron paramagnetic resonance (EPR); higher spin relaxation rates correspond to higher cluster numbers. In some bacteria, including E. coli, the additional N7 Fe-S cluster is found within the nuoG subunit. Based on their positions within the peripheral arm, it is believed that the main pathway of electron movement 
after transfer from FMN is: N3-N1b-N4-N5-N6a-N6b-N2. Due to the position of cluster N1a (and N7 in bacteria), it is not believed to be directly involved in this pathway. The midpoint redox potential difference between $\mathrm{NADH}\left(\mathrm{E}_{\mathrm{m} 7}=-320 \mathrm{mV}\right)$ and ubiquinone $\left(\mathrm{E}_{\mathrm{m} 7}=+110 \mathrm{mV}\right)$ drives electrons through this Fe-S cluster chain (a distance of more than $95 \AA$ ), and provides the energy required to pump protons for ATP production [180].

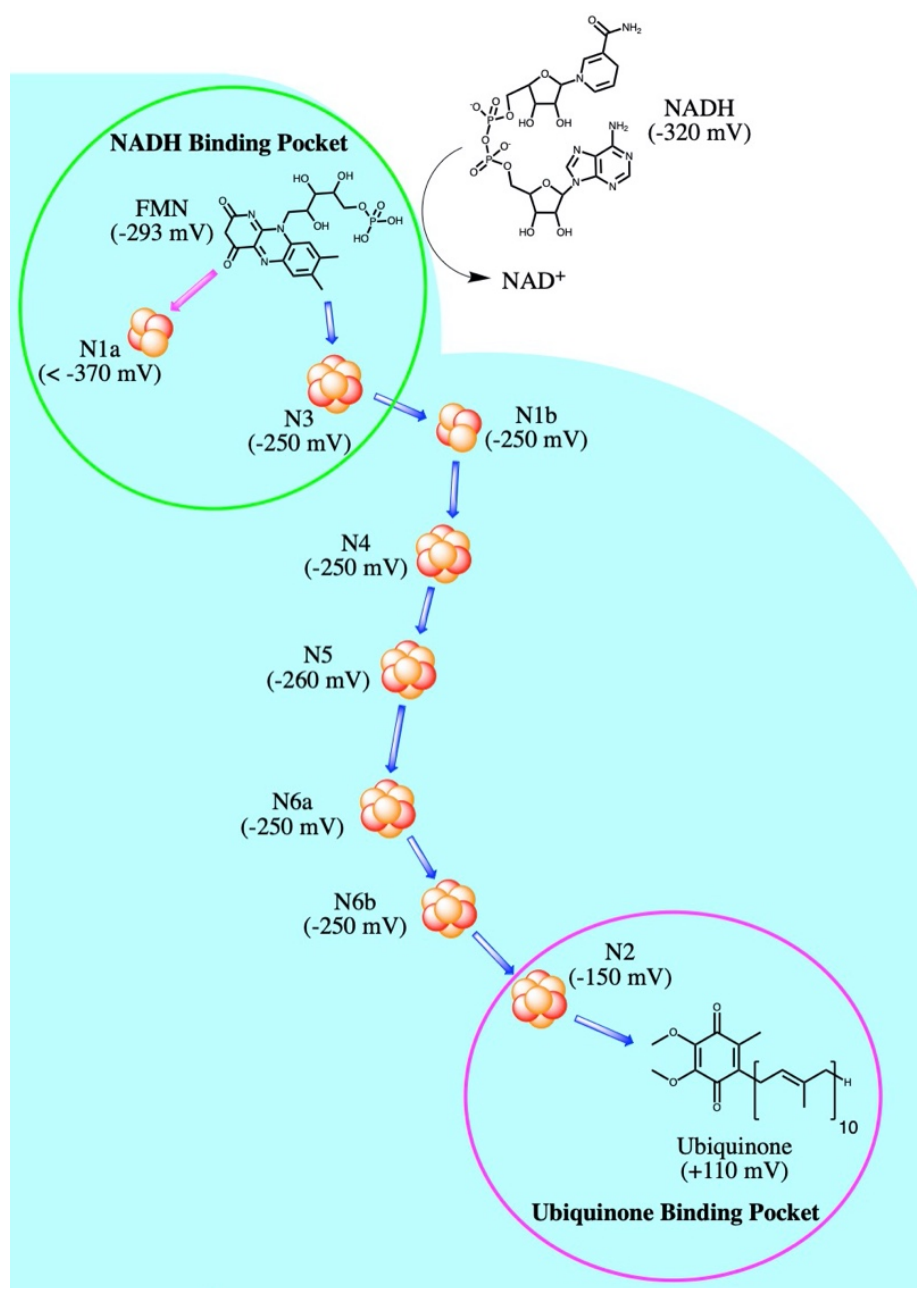

Figure $13 \mathrm{Fe}$-S chain within Complex I. The midpoint redox potential at pH of 7 (as found in B. taurus) is listed for each cluster, as well as NADH, FMN, and ubiquinone. The NADH and ubiquinone binding sites are depicted in green and magenta circles, respectively, and the main route of electron transfer is depicted by blue arrows.

The $\mathrm{N}$ module contains the NADH dehydrogenase [ubiquinone] iron-sulfur protein 1 (NDUFS1), NADH dehydrogenase [ubiquinone] flavoprotein 1 (NDUFV1), and NDUFV2 subunits and is responsible for initiating the transfer of electrons down the chain of Fe-S clusters 
via NADH and FMN [179]. The first cluster within this chain is N3, found within the N module, ligated to NDUFV1 near its $\mathrm{C}$ terminus. The N3 cluster is located $7.6 \AA$ from FMN, which is also non-covalently bound to NDUFV1 $[183,184]$. N3 receives single electrons from FMN and begins the electron transfer pathway, passing electrons to N1b. This is an intersubunit transfer, as electrons jump from NDUFV1 to NDUFS1, and is sensitive to internal water within the complex: the presence of water increases the rate of transfer by three orders of magnitude [185]. N1b, N4 and N5 are also found within the N module, bound to the NDUFS1 subunit [180, 183]. After accepting an electron from N3, electrons from N1b are passed on to N4, then to N5. Unlike the other $[4 \mathrm{Fe}-4 \mathrm{~S}]$ clusters found within Complex I, N5 is ligated by three cysteines and one histidine, resulting in slightly altered EPR properties [96].

The N1a cluster is the last remaining Fe-S cluster within the $\mathrm{N}$ module. This cluster is not believed to be a part of the main electron shuttling pathway due to its position relative to the other clusters, as well as its low redox potential $\left(E_{\mathrm{m} 7}<-370 \mathrm{mV}\right)$ compared to fully reduced FMN, flavohydroquinone $\left(\mathrm{FMNH}_{2}, \mathrm{E}_{\mathrm{m} 7}=-293 \mathrm{mV}\right)[186,187]$. However, this cluster is conserved in Complex I across taxa, suggestive of its functional significance. It has been postulated that it may prevent ROS production by reducing the lifetime of reduced FMN species. FMN is situated $7.6 \AA$ from N3 and $11.3 \AA$ from N1a [180]. After accepting two electrons simultaneously from $\mathrm{NADH}$, it is only capable of passing electrons one at a time down the Fe-S cluster chain of Complex I. It has been proposed that N1a may accept an electron from flavosemiquinone $\left(\mathrm{FMNH} \cdot, \mathrm{E}_{\mathrm{m} 7}=-389 \mathrm{mV}\right)$, shortening the lifetime of this unstable intermediate and possibly preventing electron leakage and ROS production [180]. Recently, N1a has been shown to prevent over-reduction of Complex I by stabilizing bound $\mathrm{NAD}^{+}$, thereby preventing further binding of NADH [188]. The redox state of N1a regulates NADH oxidation in 
E. coli Complex I by controlling the orientation of a peptide bond close to the NADH binding site. If the rates of $\mathrm{N} 2$ cluster oxidation are low due to a predominantly reduced quinone pool, this causes reduction of N1a by FMNH and a conformational switch within the NADH binding pocket that favours $\mathrm{NAD}^{+}$. This reduced N1a cluster thereby prevents additional binding of $\mathrm{NADH}$ and further reduction of the complex, which may provide the complex with an intramolecular feedback mechanism to prevent ROS production [188].

The Q module contains the NDUFS2, NDUFS3, NDUFS7 and NDUFS8 subunits as well as the N6a, N6b and N2 Fe-S clusters [179]. Both the N6a and N6b clusters are ligated within NDUFS8. The N6a cluster is located near the interface of the N and Q modules near the zincbinding NDUFS6 subunit, and accepts electrons from N5, passing them onwards to N6b and N2 [189]. The longest edge-to-edge distance between clusters within Complex I is the electron transfer from the N to Q modules, between N5 and N6a (14 $\AA$ ), and is believed to be a ratelimiting step within the complex. Similar to the intersubunit electron transfer between clusters $\mathrm{N} 3$ and N1b, the rate of transfer between N6b and N2 is also enhanced by the presence of molecules of water between subunits NDUFS8 and NDUFS7 [185].

The last cluster within the Q module/Complex I is the N2 cluster, ligated by four cysteine residues within the NDUFS7 subunit near its interface with NDUFS2 [183]. This cluster is located $\sim 12 \AA$ from the ubiquinone binding site, and is responsible for reducing ubiquinone to ubiquinol within the Q module of Complex I, thereby transferring electrons to downstream complexes [190]. In comparison to the other Fe-S clusters in Complex I, N2 exhibits a higher redox potential $\left(\mathrm{E}_{\mathrm{m} 7}=\sim-150 \mathrm{mV}\right)$ in most organisms, including B. taurus, $N$. crassa, and E. coli, compared to the other clusters within the Complex I cluster chain $\left(E_{\mathrm{m} 7}=\sim-250 \mathrm{mV}\right)$, making it the electron sink of the complex $[180,181,191]$. Besides it's unusually high redox potential, 
$\mathrm{N} 2$ 's reduction potential also exhibits $\mathrm{pH}$ dependence due to a hydrogen bond formed between a histidine residue in NDUFS2 $\left(\mathrm{His}^{226}\right.$ ) and the Fe-S cluster [192]. Remarkably, a similar hydrogen bond with this cluster is also observed in water-soluble [NiFe] hydrogenases in analogous subunits. Mutations at this residue within NDUFS2 to either alanine, glutamine, or cysteine, have shown to reduce the EPR signal of the cluster shifting the $\mathrm{E}_{\mathrm{m} 7}$ by $-80 \mathrm{mV}$, while only eliciting moderate effects on the catalytic activity of the complex [193]. These findings suggest that the energy conversion mechanism driving proton translocation across the IMM is not associated with one of the electron transfers between the Fe-S clusters upstream but is perhaps linked to the protonation/deprotonations of ubiquinone intermediates. Interestingly, NDUFS2 has been shown to be critical in the assembly and/or stability of the N2 cluster in Complex I, and is also implicated in oxygen-sensing mechanisms within the homeostatic oxygen-sensing system, as discussed in a later section [194].

Complex I is considered to be the main producer of reactive oxygen species within mitochondria $[195,196]$. The crystal structure of Complex I shows that the majority of redox cofactors within the enzyme are shielded from the solvent and are unlikely to react with molecular oxygen to form ROS. In Complex I, ROS production occurs at either end of the Fe-S chain, at the FMN site $\left(\mathrm{I}_{\mathrm{F}}\right)$ or the quinone binding site $\left(\mathrm{I}_{\mathrm{Q}}\right)$ and can occur when electrons travel in either the forward or reverse directions. Due to the positions of these sites within the peripheral arm, it is believed that Complex I generates ROS exclusively into the mitochondrial matrix [197].

When electrons move in the forward direction, i.e. from FMN to N2, blocking the $\mathrm{I}_{\mathrm{Q}}$ site via rotenone causes a backlog of electrons in the Fe-S cluster chain, and an over-reduction of the IF site [198]. This over-reduction of Complex I increases the lifetime of FMNH', causing 
electrons to leak and produce $\mathrm{O}_{2}{ }^{--}$. The effects of rotenone on ROS production appears to be tissue-specific, showing different responses in various cell types. Although rotenone increases ROS in renal artery smooth muscle cells (SMC), within the pulmonary artery SMC, rotenone decreases ROS production [199]. This effect is attributed to decreased overall electron flux through the complex and is explored in further detail below.

Complex I can also produce ROS via reverse electron transport (RET) [197]. RET occurs when there is an overabundance of ubiquinol in the $\mathrm{Q} / \mathrm{QH}_{2}$ pool due to reduction by electrons from Complex II. When this occurs, electrons can be transferred from ubiquinol to N2. In isolated mitochondria supplemented with succinate, RET can produce $\mathrm{NADH}$ from $\mathrm{NAD}^{+}$via RET through Complex I, however this process is also associated with a significant production of ROS, which is believed to come from the IQ site [86, 200]. Unlike the forward direction, RET is dependent on both the redox state of the $\mathrm{Q} / \mathrm{QH}_{2}$ pool as well as the proton motive force, with inhibition of Complex I by rotenone (an IQ site inhibitor) or mitochondrial uncoupling by FCCP reducing ROS production via RET.

ROS production from RET is known to be an important pathophysiological mechanism and a major contributor to oxidative stress during heart attack and stroke, via ischaemiareperfusion (I/R) injury in the heart and the brain, respectively. Ischaemia is characterized by an overabundance of fumarate which causes reversal of succinate dehydrogenase activity and accumulation of succinate. After reperfusion, the accumulated succinate is rapidly oxidized by Complex II causing increased ROS production via RET and initiating oxidative damage [200]. A new generation of antioxidants that are capable of suppressing electron leak specifically from the $\mathrm{I}_{\mathrm{Q}}$ site have been shown to protect against ischaemia-reperfusion injury in in vitro modeling of cardiac I/R, providing further evidence of RET-induced ROS production during reperfusion and 
providing new therapeutic leads for protecting against reperfusion induced oxidative damage [12].

\subsubsection{Fe-S clusters in Complex II}

Of the remaining four Fe-S clusters outside of Complex I, three are within ETC Complex II, also called succinate dehydrogenase (SDH) or succinate-coenzyme Q reductase. SDH is an IMM bound protein with a hydrophilic head that protrudes into the mitochondrial matrix. SDH serves dual functions in mitochondrial respiration: oxidizing succinate to fumarate in the TCA cycle, and reducing ubiquinone $(\mathrm{Q})$ to ubiquinol $\left(\mathrm{QH}_{2}\right)$ as a result of electron transfer from $\mathrm{FADH}_{2}$ in the ETC (Figure 4) [201]. Complex II is the only ETC complex without any mtDNAencoded subunits, and is comprised of four nuclear-encoded core proteins: the flavoprotein (SDHA) containing a covalently attached flavin adenine dinucleotide (FAD) cofactor, an Fe-S protein (SDHB) housing the clusters required for electron transfer from $\mathrm{FADH}_{2}$ to ubiquinone, and two hydrophobic membrane-spanning subunits (SDHC and SDHD) which anchor the catalytic SDHA-SDHB dimer to the IMM and contain the ubiquinone binding site [202]. Within the SDHB subunit, three Fe-S clusters exist, all in different forms: [2Fe-2S], [4Fe-4S], and [3Fe$4 \mathrm{~S}]$. The rhombic cluster is found in the N-terminal domain of SDHB, adjacent to the FAD bound to SDHA, whereas the other two clusters are found within the C-terminal domain [203]. All three clusters are aligned nearly linearly, each spaced less than $14 \AA$ from the other (the distance limit for productive electron transfer) [203, 204]. The first cluster in this chain [2Fe-2S] accepts electrons from FAD, passing them through the [4Fe-4S] cluster and finally to [3Fe-4S], located 7.1 Å from the ubiquinone binding site [203].

Mitochondrial SDH also contains a fifth redox prosthetic group, heme b, located between subunits SDHC and SDHD. Although this heme was originally believed to transfer electrons 
from the $[3 \mathrm{Fe}-4 \mathrm{~S}]$ group to ubiquinone, the edge-to-edge distance between the [3Fe-4S] cluster and bound ubiquinone is smaller (7.1 $\AA$ ) than the distance between the cluster and the heme (13.3 $\AA)$. Additionally, the redox potential of heme $b\left(E_{m 7}=-185 \mathrm{mV}\right)$ is much lower than both the $[3 \mathrm{Fe}-4 \mathrm{~S}]$ cluster $\left(\mathrm{E}_{\mathrm{m} 7}=+60 \mathrm{mV}\right)$ and the ubiquinone reduction $\left(\mathrm{E}_{\mathrm{m} 7}=+110 \mathrm{mV}\right)$, suggesting transfer from the cluster directly to heme b would be unfavourable [203]. In E. coli Complex II, the heme group is closer to both the $[3 \mathrm{Fe}-4 \mathrm{~S}]$ cluster and bound quinone, and also has a more comparable redox potential $\left(\mathrm{E}_{\mathrm{m} 7}=+36 \mathrm{mV}\right)$ [205]. It is believed that electrons transferred to ubiquinone are in equilibrium with heme $b$, an effect which may stabilize ubisemiquinone radicals and reduce production of ROS from the enzyme.

Under normal conditions, production of ROS by Complex II is considered to be negligible, although mutated Complex II is known to cause oxidative stress due to ROS production at the flavin site $\left(\mathrm{II}_{\mathrm{F}}\right)$ [206-208]. In isolated mitochondria, Complex II is capable of producing ROS at $\mathrm{II}_{\mathrm{F}}$, as the FAD within the flavoprotein is known to be a potent site of electron leak [86]. Similar to Complex I, ROS production by Complex II occurs exclusively in the matrix, and this electron leakage can result from electrons traveling in the forward direction after being donated by succinate/FADH 2 , or in the reverse direction after transfer from ubiquinol [51]. ROS production at site $\mathrm{II}_{\mathrm{F}}$ is dependent on the occupancy of the substrate oxidation site, with significant ROS production only occurring in the presence of an open flavin site. Occupiers of this site, including the dicarboxylic acids oxaloacetate and malate, as well as succinate itself, are postulated to bind at site $\mathrm{II}_{\mathrm{F}}$, preventing oxygen from entering and being reduced to produce ROS in vivo [51, 209].

\subsubsection{Fe-S clusters in Complex III}


Electrons are eventually shuttled from Complexes I and II via $\mathrm{QH}_{2}$, to Complex III, where the final Fe-S cluster within the ETC is found. Complex III, also called cytochrome bc-1 complex or coenzyme Q: cytochrome c-oxidoreductase, is a homodimer containing three essential redox subunits: cytochrome $\mathrm{b}$, cytochrome $\mathrm{c}_{1}$, and the iron sulfur protein (ISP) containing the sole Fe-S cluster [210]. Although in its simplest form this complex exists with only these three essential subunits, in bovine and humans, this complex includes an additional 8 supernumerary subunits in each monomer which are believed to contributed to the structure's stability [211-213]. At this complex, ubiquinol is oxidized in a bifurcated fashion (commonly known as the Q-cycle) at the $\mathrm{Q}_{\mathrm{o}}$ site, where two electrons from $\mathrm{QH}_{2}$ are passed through two separate pathways (Figure 14) [213]. The "low potential pathway" involves electrons passing through two heme units within cytochrome b: a low potential heme cyt $b_{\mathrm{L}}\left(\mathrm{E}_{\mathrm{m} 7}=-30 \mathrm{mV}\right)$, and a high potential heme cyt $b_{H}\left(E_{m 7}=+100 \mathrm{mV}\right)$ [213]. This electron will either reduce ubiquinone to ubisemiquinone (SQ), or after a subsequent round of this bifurcated reaction, reduce SQ to $\mathrm{QH}_{2}$ at the quinone reductase site $\left(\mathrm{Q}_{\mathrm{i}}\right)$. This low potential pathway therefore regenerates a molecule of ubiquinol every two rounds of ubiquinone oxidation at the $\mathrm{Q}_{\mathrm{o}}$ site, with uptake of two protons from the mitochondrial matrix [214]. 


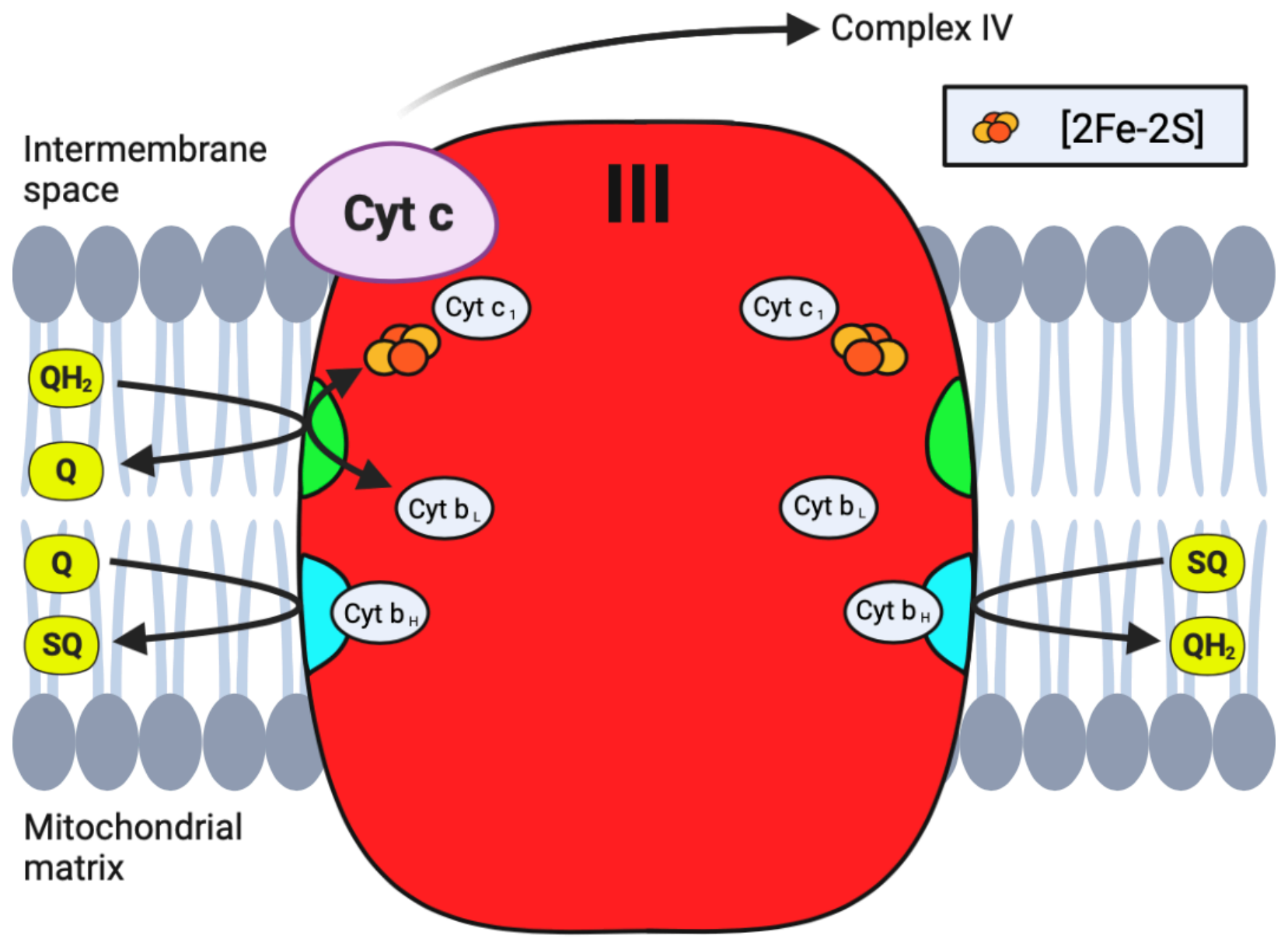

Figure 14 Q-cycle within Complex III. Ubiquinol $\left(Q_{2}\right)$ is oxidized at the $Q_{o}$ site (shown in green), passing electrons to either cytochrome c (cyt c) through the [2Fe-2S] cluster within the ISP and cytochrome $c_{1}\left(c y t c_{1}\right)$, or to the $Q_{i}$ site (shown in blue) through cyt $b_{L}$ and $b_{H}$. As shown on the left side of the complex, ubiquinone $(Q)$ is reduced to ubisemiquinone $(S Q)$ at the $Q_{i}$ site after a single round of the $Q$-cycle, while a second round of the cycle reduces $S Q$ to $Q_{2}$, as shown on the right side of the complex.

The "high potential pathway" involves electron transfer from ubiquinone at the $\mathrm{Q}_{\mathrm{o}}$ site to cytochrome c, via the Fe-S cluster within the iron sulfur protein $\left(E_{\mathrm{m} 7}=+250 \mathrm{mV}\right)$ and cytochrome $\mathrm{c}_{1}\left(\mathrm{E}_{\mathrm{m} 7}=+230 \mathrm{mV}\right)$ [213]. In contrast to other clusters found within the respiration complexes, the cluster within ISP has a Rieske-type [2Fe-2S] structure, where the iron atoms are coordinated by two cysteine and 2 histidine residues (Figure 1) [215]. Both ISP and cytochrome $\mathrm{c}_{1}$ are anchored to the IMM via transmembrane helices, and both contain soluble extramembrane domains within the IMS $[211,216]$. This extramembrane domain of ISP (ISP-ED) contains the Rieske Fe-S cluster within the IMS, and moves between the $\mathrm{Q}_{\mathrm{o}}$ site and cytochrome $\mathrm{c}_{1}$ to 
facilitate electron transfer through Complex III [217]. Although substantial conformational change within ISP-ED is required for the movement between these redox centers, the exact mechanism is still unknown, although different models have been proposed to explain its movement [218, 219].

Aside from Complex I, Complex III is the other primary site of mitochondrial ROS production. The $\mathrm{Q}_{\mathrm{o}}$ site is known to be the major source of ROS production by Complex III [86]. The production of ubisemiquinone radicals associated with the bifurcated nature of electron movement at this complex increases its susceptibility to generate ROS. The $\mathrm{Q}_{\mathrm{i}}$ site inhibitor antimycin A prevents electron transfer from the $\mathrm{Q}_{o}$ site, increasing the lifetime of the ubisemiquinone species which readily reacts with oxygen to form $\mathrm{O}_{2} \cdot{ }^{-}$[220]. Inhibition of Complex III by antimycin A also increases the $\mathrm{QH}_{2} / \mathrm{Q}$ ratio, which can lead to electron backlog in Complexes I and II. Similar to the effects of rotenone on Complex I, the effects of antimycin A on Complex III also appear to be tissue-specific, causing opposing changes in ROS production in renal and pulmonary artery SMC. [61] In the rat lungs and pulmonary artery SMC, antimycin A decreases ROS production, an effect attributed to decrease electron flux through the complex. The tissue-specific effects of proximal ETC inhibitors rotenone and antimycin on ROS production are discussed in more detail in the next section [73]. Unlike both Complexes I and II, Complex III releases $\mathrm{O}_{2} \cdot-$ into both the IMS as well as the mitochondrial matrix [221].

\subsection{Fe-S clusters and the Homeostatic Oxygen-Sensing System}

Electron tunneling through the ETC complexes and the associated ROS production that occurs at several distinct sites has been implicated in both pathophysiological diseases and normal physiological processes. Although the sites of electron leak are typically attributed to the flavin sites or quinone binding sites within the respiratory complexes, the role of Fe-S clusters in 
ROS production and signaling is a novel and emerging area of investigation, and in recent years has been shown to be important within oxygen-sensing systems. Changes in oxygen tension are accompanied by redox changes, with Fe-S clusters being highly susceptible to redox changes and known to function as redox switches within cells $[222,223]$. This is of particular interest in the highly specialized tissues designed to sense and respond to changes in oxygen. The homeostatic oxygen-sensing system (HOSS), outlined in Figure 15, is comprised of specialized tissues throughout the body, specifically adapted to sense small changes in both arterial oxygen content and airway oxygen levels [90]. In response to changes in environmental oxygen levels or localized changes in $\mathrm{pO}_{2}$, these tissues can elicit changes in vascular tone, respiration, and neurosecretion [30]. The chromaffin cells of the fetal adrenal medulla, the type 1 cells within the carotid body, as well as the neuroepithelial bodies within the lungs, are all members of the HOSS which respond to changes in oxygen via transmitter release. Also included within HOSS are the smooth muscle cells (SMC) of the fetoplacental arteries, the ductus arteriosus, and the systemic and resistance pulmonary arteries (PAs), all of which respond to changes in oxygen content by modulating vascular tone $[1,90]$. In all of these tissues, the cellular response to changes in oxygen tension are mediated by changes in ROS production, largely by the mitochondria. The modulation of redox sensitive $\mathrm{K}^{+}$channels causes changes in membrane polarization and influences $\mathrm{Ca}^{2+}$ entry into the cell $[1,224,225]$. This is followed either by changes in vascular tone or secretion of transmitters, depending on the tissue type. 


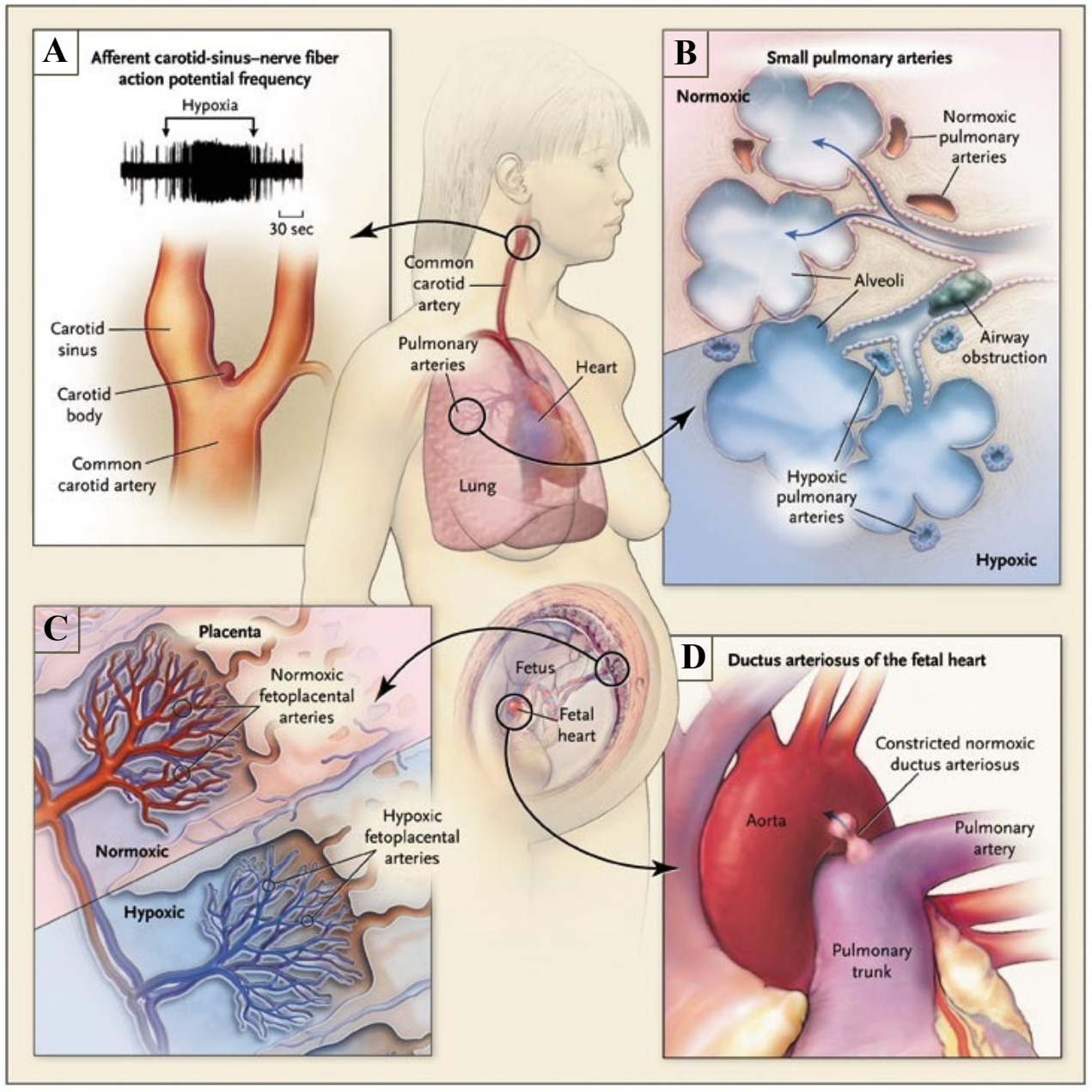

Figure 15 The specialized tissues of the Homoeostatic Oxygen Sensing System (HOSS). A. The carotid body, (located at the bifurcation of the carotid artery) increases the frequency of action potentials upon exposure to hypoxia. B. Pulmonary arteries constrict in hypoxia to divert blood to regions of the lung with better oxygenation (ventilation-perfusion matching). C. Hypoxic Fetoplacental vasoconstriction optimizes maternal and fetal perfusion matching. D. The ductus arteriosus rapidly constricts in response to normoxia postnatally, properly separating the pulmonary and systemic circulations. Adapted with permission from Weir et al. (2005) NEJM [90].

In response to hypoxia, the pulmonary arteries constrict, whereas the renal arteries (RAs) dilate. In a study by Michelakis et al., diversity in mitochondrial function was proposed as a possible explanation for the observed differences in PAs and RAs responding to hypoxia. Several differences are observed in mitochondria between these tissues, with lung mitochondria being more depolarized, having lower oxygen consumption rates, and producing more ROS at baseline [199]. Proximal ETC inhibitors, like rotenone (an inhibitor of Complex I) and antimycin A (an inhibitor of Complex III), were found to mimic hypoxia in both tissues, causing inhibition of $\mathrm{K}^{+}$ 
channels in PAs and activation of $\mathrm{K}^{+}$channels in RAs. Interestingly, other modulators of vascular tone in these vessels, such as endothelin-1 and 4-AP (a Kv blocker), constrict both vessel types, suggesting the mechanism behind the opposing effects of hypoxia on both tissue types may reside in the mitochondrial ETC, rather than in downstream ion channels [199]. These effects of hypoxia on these vessels were also observed in the absence of endothelium, suggesting the sensor of oxygen may reside within the SMC themselves.

As electrons flow through the IMM, electron leakage occurs at several sites and upon exiting the ETC, electrons can combine with molecular oxygen to produce $\mathrm{O}_{2} \cdot{ }^{-}$radicals [226]. These anions are quickly converted into $\mathrm{H}_{2} \mathrm{O}_{2}$, a species with a larger diffusion radius, via the actions of superoxide dismutase [227]. $\mathrm{H}_{2} \mathrm{O}_{2}$ is a well-established signalling molecule, and has the ability to cause numerous structural/functional changes within proteins, including oxidizing methionine residues, and forming disulfide bridges via oxidation of cysteine residues [228]. Among these redox-sensitive proteins are ion channels, such as the potassium channel Kv1.5; changes in redox state of the cell changes the open state probability of the $\mathrm{K}^{+}$channel, thus controlling membrane potential and in-turn modulating certain voltage-sensitive channels (e.g. L-type $\mathrm{Ca}^{2+}$ channels) $[229,230]$. Redox changes also modulate activation of Rho-kinase which, when active, maintains SMC constriction independent of intracellular calcium [90, 231, 232]. In this way, it is believed that alterations in electron leak and ROS production may provide a sensing mechanism whereby changes in rates of mitochondrial respiration (dependent on oxygen availability), alter the redox status of the cell, and modulate downstream ion channels. Recent findings suggest that ROS production from Fe-S clusters plays an important role in redox signaling within oxygen sensitive tissues. These changes in ROS production may also alter the Fe-S clusters themselves, with reversible redox changes to Fe-S clusters potentially playing a 
vital role in sensing changes in oxygen tension. The role of Fe-S clusters and the effects of ROS signaling in the fetoplacental arteries, carotid body, pulmonary arteries, and ductus arteriosus will be explored in the following sections.

\subsubsection{Oxygen-sensing in fetoplacental arteries}

The fetoplacental arteries are highly sensitive to changes in oxygen tension [233]. The placental cotyledons contain the fetal capillaries and are surrounded by maternal blood, thus serving as the site of exchange for nutrients and oxygen from the maternal blood to fetal circulation. Fetoplacental arteries constrict under hypoxic conditions as a result of hypoxic inhibition of Kv channels, similarly to the PAs [234]. Distribution of maternal blood flow is variable amongst placental cotyledons, with hypoxic fetoplacental artery vasoconstriction acting to divert fetal blood flow to better perfused cotyledons to increase efficiency of placental oxygen exchange [234, 235]. The role of mitochondria and mitochondrial Fe-S clusters in fetoplacental artery oxygen-sensing requires further investigation. However, while the acute placental response to oxygen has yet to be studied, chronic hypoxia of the placenta from under-perfusion is a key pathologic feature of pre-eclampsia $[236,237]$. Pre-eclampsia occurs in $3-8 \%$ of all pregnancies [238]; it can present as a maternal syndrome (hypertension and proteinuria), or as a fetal syndrome (fetal growth restriction, abnormal oxygenation, reduced amniotic fluid), and is associated with increased maternal and perinatal morbidity and mortality [236, 238, 239]. Preeclampsia occurs more frequently at high altitudes [240, 241], with high altitude pregnancies having lower levels of Complex I and ISCU in placental tissue [242]. Additionally mitochondrial dysfunction has been reported in pre-eclampsia, with decreased expression of ISCU [236]: the transcription factor HIF-1 (hypoxia inducible factor 1 ) acts to decrease ISCU via the HIF-1aresponsive miR210 [236, 242]. Though the role of mitochondrial Fe-S clusters in the placental 
oxygen response has yet to be studied, the modulation of an essential Fe-S cluster assembly pathway component by hypoxia suggests that the role of Fe-S clusters in placental oxygensensing is a valuable area of further investigation.

\subsubsection{Oxygen-sensing in the carotid body}

Acute oxygen-sensing by chemoreceptors within the carotid body is essential for mammalian adaptations to changing $\mathrm{pO}_{2}$ levels, as occurs in response to disease and to changes in atmospheric oxygen supply with altitude. In response to decreased blood oxygen, activation of chemoreceptors within the carotid body glomus cells mediates cardiorespiratory reflexes, causing both sympathetic activation and hyperventilation. Within the glomus cells, $\mathrm{O}_{2}$-sensitive $\mathrm{K}^{+}$channels close in response to hypoxia, causing subsequent cell depolarization and release of neurotransmitters which activate sensory fibers that terminate at the respiratory center within the brain stem [229, 243, 244]. Fernández-Agüera et al. demonstrated that ablation of the Ndufs2 gene within the carotid body glomus cells of mice results in the loss of the hypoxic ventilatory response, while maintaining responses to other stimuli such as hypercapnia and hypoglycemia [78]. Using this same model of knockout mice, deletion of Ndufs4 was observed to reduce Complex I activity, while maintaining normal cellular responses to hypoxia, suggesting an important role for Ndufs 2 and ubiquinone oxidoreductase activity in oxygen-sensing within glomus cells. In this model of Ndufs2 deficient mice, glomus cells exhibited increased levels of $\mathrm{NADH}$ and ROS, suggesting the loss of the hypoxia response within these cells may be a result of alterations in mitochondrial homeostasis, rather than a consequence of Complex I dysfunction specifically. To address this, Ndufs2-null cells were supplemented with pyruvate and $\alpha$ ketoglutarate to regenerate $\mathrm{NAD}^{+}$, as well as exogenous succinate to bolster the Complex II through Complex IV pathway. Despite these supplements, these cells were still found to exhibit 
an almost complete absence of hypoxic response, suggesting an essential role of Complex I derived NADH and ROS in acute oxygen-sensing [245]. This same group also found that in response to hypoxia, glomus cells showed an increase in ROS production in the IMS and a decrease in ROS production within the mitochondrial matrix. One could speculate that this may be the result of $\mathrm{O}_{2} \cdot-$ being electrostatically channeled into the IMS by its positive potential, causing the subcellular compartmentalization observed [245]. The authors suggest that during hypoxia, an increase in ratio of reduced to oxidized ubiquinone may cause decrease rates of electron flux through Complex I, possibly increasing the lifetime of the reduced form of the N2 cluster, causing increased electron leak and $\mathrm{O}_{2} \cdot{ }^{-}$production. Although not speculated by the authors, these findings may also suggest that alterations in the redox midpoint potential of the N2 cluster due to the absence of the hydrogen bond formed between the cluster and Ndufs 2 may cause less controlled movement of electrons through the Fe-S chain, possibly increasing the chances of leakage and ROS production.

\subsubsection{Oxygen-sensing in the pulmonary artery}

Oxygen-sensing within the pulmonary arteries tightly matches ventilation to perfusion, optimizing oxygen uptake and systemic delivery [90]. In response to hypoxia, small resistance pulmonary arteries constrict, diverting blood away from poorly ventilated portions of the lung and towards better oxygenated portions, optimizing overall oxygen uptake [246]. This hypoxic pulmonary vasoconstriction (HPV) mechanism is intrinsic to the SMC of the resistance pulmonary arteries, and similar to other tissues included in the HOSS, involves the coordination of redox and voltage sensitive $\mathrm{K}^{+}$and $\mathrm{Ca}^{2+}$ ion channels. In response to hypoxia, inhibition of $\mathrm{K}^{+}$ channels leads to cell depolarization, $\mathrm{Ca}^{2+}$ uptake through large conductance voltage sensitive channels, and subsequent vasoconstriction in response to increased intracellular $\mathrm{Ca}^{2+}[247]$. 
Although like other members of the HOSS the sensor responsible for this cascade is believed to reside in the mitochondria, the exact mechanism of oxygen-sensing, as well as the influence of hypoxia on ROS production within pulmonary artery SMC (PASMC), is still a matter of debate $[56,59-61,65,66,68,248]$. Similar to the findings in the carotid body glomus cells, a recent study by our group has shown that the HPV response within PASMC is also dependent on Ndufs2 [77]. Ndufs2 expression was observed to be greater in rat PASMC in comparison to renal artery SMC, providing a possible reasoning for the discrepancy in oxygen-sensing abilities between these cell types as described by Michelakis et al. [199]. In isolated PASMC treated with siRNA to knockdown Ndufs2, the hypoxia induced rise in intracellular $\mathrm{Ca}^{2+}$ was not observed, whereas responses to other stimuli such as hyperkalemia remained intact. siNdufs2 was also observed to mimic aspects of chronic normoxia, including decreased Complex I activity, elevated NADH/NAD ${ }^{+}$ratios, and decreased expression of the $\mathrm{O}_{2}$-sensitive $\mathrm{Kv} 1.5$ channel. Nebulization of siNdufs 2 resulted in partial knockdown of subunit expression ( $\sim 35 \%$ knockdown in whole lung homogenate), but completely eliminated HPV in a rat model. Similarly, an attenuated HPV response was observed in mice treated with siNdufs2 as measured using intravital microscopy and confocal imaging of excised lungs. Collectively, these in vitro and in vivo findings suggest a key role for this subunit in the oxygen-sensing pathway within the resistance pulmonary arteries. As in the carotid body, these findings further suggest an importance of the interaction between Ndufs2 and the N2 Fe-S cluster, and suggest that the redox midpoint potential of this cluster is vital for maintaining normal oxygen signaling pathways through the control of ROS production from Complex I. 
Normoxia

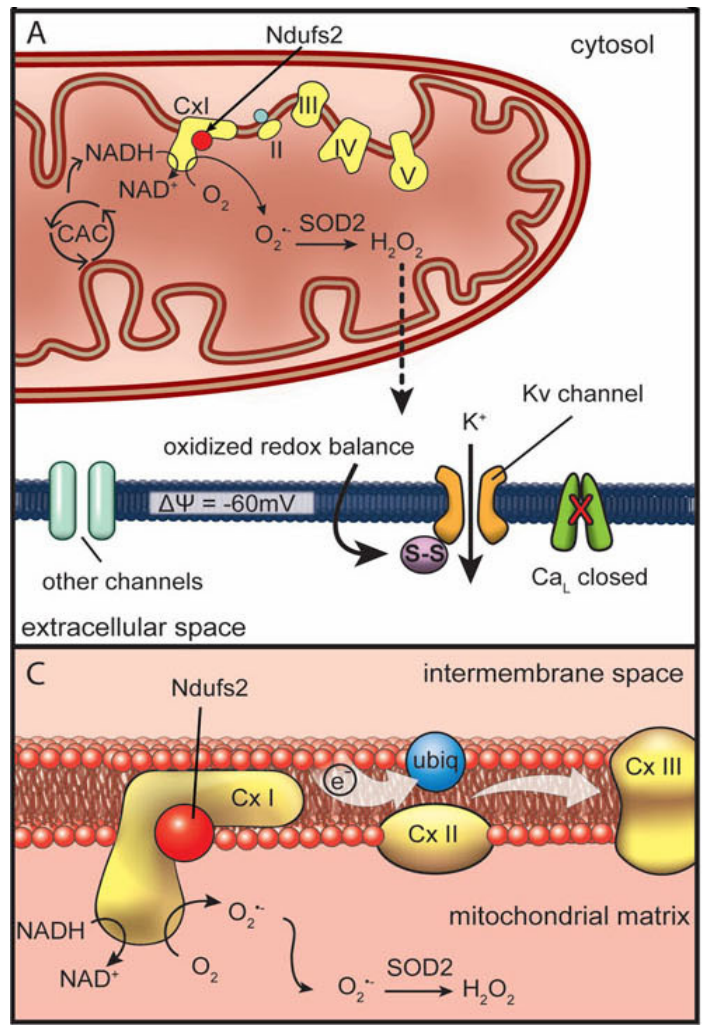

Hypoxia

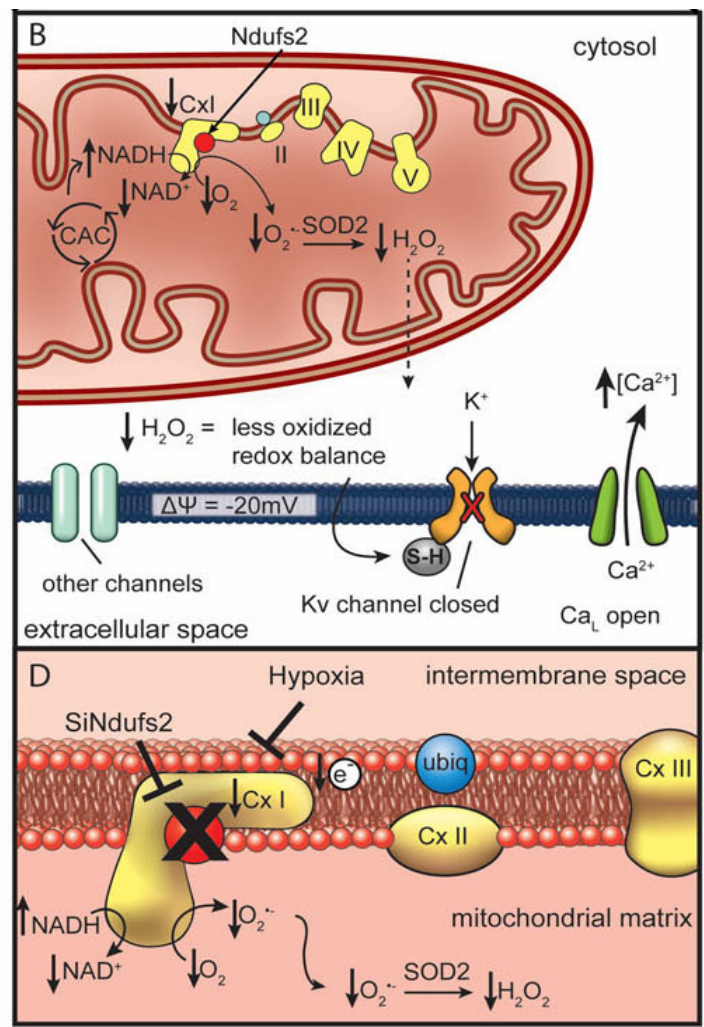

Figure 16 PASMC mitochondrion during normoxia and hypoxia. A. Under normoxic conditions, oxygen radicals produced at ETC Complex I are converted to $\mathrm{H}_{2} \mathrm{O}_{2}$ by SOD2, with this ROS production leading to oxidation of sulfhydryl groups in the Kv channel, forming disulfide bridges $(S-S)$ and increasing the channel's open-state probability. B. With a switch to hypoxia, production of superoxide is decreased due to decreased uncoupled electron transport. The resultant decrease in $\mathrm{H}_{2} \mathrm{O}_{2}$ and the accumulation of NADH depolarize the cell, closing the Kv channels, with subsequent opening of calcium channels causing vasoconstriction. C. Optimal function of Complex I requires intact Ndufs2. D. Inhibition of Complex I, via hypoxia or siRNA targeting Ndufs2, results in a more reduced redox state. Adapted with permission from Dunham-Snary et al. (2019) Circ. Res. [77].

7.5.4 Oxygen-sensing in the ductus arteriosus

Oxygen-sensing in the ductus arteriosus (DA) is crucial for circulatory transition into neonatal life. The ductus arteriosus is a fetal vessel connecting the pulmonary artery and aorta, diverting blood in the pulmonary circulation, oxygenated by the placenta, away from the developing lungs to the systemic circulation. The DA rapidly constricts in response to the changes in the arterial partial pressure of oxygen $\left(\mathrm{PaO}_{2}\right)$ that occur with the transition from the hypoxic fetal environment $\left(\mathrm{PaO}_{2}<40 \mathrm{mmHg}\right)$ to the normoxic neonatal environment $\left(\mathrm{PaO}_{2} \sim 100\right.$ mmHg) [249]. The immediate constriction of the DA, occurring within minutes of birth, and 
permanent closure, which typically occurs within 72 hours, is crucial for proper separation of the pulmonary and systemic circulation [250,251]. Failure of DA closure is a common complication of extreme preterm birth and can have serious adverse outcomes if not treated [249, 252]. DA response to oxygen occurs via an oxygen-induced increase in mitochondrial ROS in DA smooth muscle cells, with ROS inhibiting voltage-gated potassium channels (Kv) [9, 253, 254]. Kv channel inhibition leads to cell depolarization, opening of L-type $\mathrm{Ca}^{2+}$ channels, and smooth muscle cell constriction [255]. Mitochondrial ROS are mainly produced by Complexes I and III of the ETC, with inhibition of these complexes inhibiting DA response to oxygen without affecting constriction induced by vasoconstrictors [8]. A recent transcriptomic study by Bentley et al [256] explored the gene expression profiles of human DA smooth muscle cells under hypoxia and following 96 hours oxygen exposure. This study found that, among genes significantly differentially expressed between the oxygen condition, mitochondrial pathways were highly enriched [256]. Furthermore, 20 genes from Complex I were significantly upregulated by oxygen exposure [256]. While transcriptomic data cannot shed light on the mechanistic pathways involved, these findings point to a role of Complex I in the oxygen response of DA smooth muscle cells. The specific role of Complex I subunits and Fe-S clusters is a promising area that requires further investigation as the DA is a historically under researched model of oxygen-sensing.

\subsection{Fe-S clusters as emerging targets of therapeutics}

Fe-S clusters play important roles in a variety of cellular functions, and as such have increasingly been of interest in drug research, either as potential therapeutic targets or as the source of drug toxicity that can be mitigated. Fe-S clusters can be the subject of therapeutic targeting in three primary domains: targeting of Fe-S clusters within a protein, targeting Fe-S 
cluster biogenesis, and drug-induced ROS production modulating Fe-S clusters (reviewed in detail by Vernis et al. [257]). Fe-S clusters frequently play a critical role in the catalytic subunits of proteins and are highly sensitive to redox changes, making them useful potential targets that could have large effects [222]. Fe-S clusters are particularly sensitive to redox changes within the cell, and play important roles serving as redox switches, sensing changes in ROS [222, 223]. Redox changes can cause proteins to lose their cluster, interconvert between $[4 \mathrm{Fe}-4 \mathrm{~S}]$ and $[2 \mathrm{Fe}-$ 2S], or change the redox state of the cluster by giving or receiving an electron [223]. Proteins using Fe-S redox switches are found in bacteria, yeast, and mammals, and are involved in a variety of functions, including: enzyme protection, Fe-S cluster transfer/repair (mitoNEET), DNA repair, and regulation of gene expression [223]. MitoNEET is an Fe-S protein anchored to the outer mitochondrial membrane [258]. MitoNEET, a homodimer with a [2Fe-2S] cluster in each monomer, plays a role in Fe-S cluster shuttling and in redox reactions [258-260]. As a general rule, when the Fe-S cluster is reduced, the protein is in a dormant state, with a switch to active state occurring when a signal induces oxidation of the cluster [100, 223, 261].

\begin{tabular}{l|l|l} 
Drug & Target & Effect \\
\hline PEITC & Glutathione & $\begin{array}{l}\text { Glutathione depletion increases ROS, degrading Fe-S cluster of NADH } \\
\text { Dehydrogenase 3 [262] }\end{array}$ \\
\hline Cluvenone & $\begin{array}{l}\text { MitoNEET \& NAF- } \\
1\end{array}$ & $\begin{array}{l}\text { Cluvenone-derivative MAD28 destabilizes Fe-S clusters of mitoNEET and } \\
\text { NAF-1, inhibiting cell proliferation, targeting cancer cells [263-266] }\end{array}$ \\
\hline Pioglitazone & MitoNEET & $\begin{array}{l}\text { Stabilizes Fe-S clusters and protects from damaging effects of NADP(H) } \\
\text { binding [267-269] }\end{array}$ \\
\hline $\begin{array}{l}\text { Primaquine } \\
\text { L-serine }+ \\
\text { fluoroquinolones }\end{array}$ & $\begin{array}{l}\text { Aconitase \& Rli1 } \\
\text { clusters }\end{array}$ & $\begin{array}{l}\text { Targeting Fe-S clusters decreases activity of aconitase and RNase L inhibitor } \\
\text { (Rli1), with inhibitory effect on respiration and growth of malaria parasites } \\
\text { [270] }\end{array}$ \\
\hline '882 & $\begin{array}{l}\text { Increases ROS production, destabilizing Fe-S clusters. Combination of L- } \\
\text { serine with ofloxacin or moxifloxacin increases bactericidal efficiency [271- } \\
273]\end{array}$ \\
\hline complex
\end{tabular}




\begin{tabular}{l|l|l}
\hline- & SUF & $\begin{array}{l}\text { Malaria parasites necessitate Fe-S clusters as cofactors: targeting SUF Fe-S } \\
\text { cluster biosynthesis for potential anti-malarial drugs [275-278] }\end{array}$ \\
\hline Doxorubicin & Increased ROS & $\begin{array}{l}\text { Cardiac toxicity of the anticancer drug results from iron-mediated } \\
\text { mitochondrial ROS production [279-282]. Adjuvant therapies can modulate } \\
\text { adverse effects [283-285] }\end{array}$ \\
Table 2. Summary of drugs targeting Fe-S clusters and their mechanisms of action.
\end{tabular}

Drugs acting on Fe-S targets either directly or indirectly have been shown to be effective in a wide variety of treatments (outlined in Table 2), from anticancer drugs to antibacterial/antiparasitic drugs. One such drug is -phenethyl isothiocyanate (PEITC), a natural anticancer product highly effective against human leukemia [262]. PEITC increases production of ROS with depletion of mitochondrial antioxidant glutathione [262]. At least part of the anticancer activity of PEITC is due to this increase in ROS degrading the Fe-S center of NDUFS3 of ETC Complex I [257, 262].

Cluvenone is a mitochondria-targeted molecule, which has demonstrated anticancer properties and decent tumor selectivity [286]. MAD-28, a cluvenone derivative, acts by binding and destabilizing two [2Fe-2S] proteins overexpressed in several cancer lines: mitoNEET (mitochondria), and nutrient deprivation autophagy factor-1 (NAF-1, endoplasmic reticulum) [263-265]. MAD-28 destabilizes the Fe-S clusters by breaking the coordinative bond between the histidine ligand and the iron of the mitoNEET and NAF-1 Fe-S clusters [263, 266]. Due to this cluster destabilization, MAD-28 strongly inhibits cell proliferation and has high specificity in targeting cancer cells [263, 266, 287, 288].

In addition to its overexpression in cancer lines, mitoNEET has been implicated in diabetes and is the target of the thiazolidinedione class of insulin-sensitizing drugs such as pioglitazone $[269,289,290]$. MitoNEET primarily interacts with NADP(H), with NADP(H) binding destabilizing the Fe-S cluster and facilitating cluster release [268]. Pioglitazone acts to 
enhance the stability of mitoNEET Fe-S clusters [267], binding with a much higher affinity than NADP(H) and protecting the cells from damaging effects [268].

There are a number of anti-bacterial and antiparasitic drugs that function through Fe-S cluster targeting. Fe-S clusters are targeted by the antimalarial drug Primaquine, as shown in yeast [270]. Primaquine decreases the activity of aconitase and RNase L inhibitor (Rli1), both of which are sensitive to oxidative damage due to their reliance on Fe-S clusters for activity [270]. Rli1 is an essential protein involved in a variety of cellular processes, including ribosome biogenesis [291] and translation initiation and termination [292, 293]. The Fe-S clusters of aconitase and Rli1 are proposed to be the primary target of primaquine in vivo, with the growth inhibitory effect reliant on respiration and the subsequent ROS production [270].

Another antibacterial treatment of interest is the combination of L-serine with the two fluoroquinolones ofloxacin or moxifloxacin, with combined treatment having higher bactericidal efficiency regardless of growth phase $[257,271,272]$. These effects are due to an increase in ROS production and rapid disruption of Fe-S clusters, though whether the clusters are directly targeted in addition to their redox-mediated disruption was not explored [273].

The examination of novel antimicrobial strategies has become increasingly vital as antibiotic resistance emerges. This was the focus of a study by Choby et al. [274] where the attempt to circumvent antimicrobial resistance in Staphylococcus aureus strains identified a new molecule named ' 882 . The toxicity of ' 882 was due to its inhibition of the sulfur mobilization (SUF) biosynthesis complex. The SUF operon encodes six major proteins that function in Fe-S cluster assembly (SufB, SufC, SufD), with sulfur mobilization from cysteine mediated by SufE and SufS, and with the use of carrier protein SufA [135, 294-297]. ' 882 physically interacts with 
the SUF Fe-S cluster synthesis machinery and subsequently decreases the activity of aconitase, an Fe-S cluster-dependent enzyme [274].

The SUF Fe-S synthesis pathway has also been suggested as a promising target in malaria parasites. Malaria parasites contain a plastid organelle called the apicoplast which "harbors biochemical pathways of prokaryotic origin" [275], thought to have arisen from a separate endosymbiotic event [298]. The apicoplast is necessary for survival of malaria parasites during the liver and blood stages of development [276]. Apicoplast enzymes are predicted to necessitate Fe-S cluster cofactors [277, 299-302] and the SUF pathways play a crucial role in maintaining the apicoplast [276], thus the SUF pathway is a promising target for potential antimalarial treatments. This idea has been extended to suggestions that the SUF pathway and apicoplast proteins are promising drug targets for anti-parasitic drugs against apicomplexan parasites in general, as these proteins are "among the most divergent relative to the mammalian proteins" [278] and thus could likely be tolerated by humans. This target/pathway is included in Table 2, though a named drug has yet to be developed.

The targeting of Fe-S clusters has also been found as the cause for adverse outcomes from certain drugs. For example, doxorubicin is an anticancer drug of the anthracycline family [303] that can also lead to development of cardiac toxicity and cardiomyopathy [304, 305]. The accumulation of doxorubicin in mitochondria can initiate mitochondrial production of ROS and RNS [306], activating apoptotic pathways [282, 307]. Additionally, it has been proposed that an increase in ROS is induced by doxorubicin in an iron-mediated pathway [279-281, 308].

Doxorubicin functions as an iron chelator, with the resultant complex between iron and the drug catalyzing conversion of hydrogen peroxide to hydroxyl radicals [281]. With a cardioprotective adjuvant treatment such as dexrazoxane, which chelates free iron [283], the doxorubicin-induced 
depletion of mitochondrial DNA due to oxidative stress can be avoided [284]. Other mitochondrial targeted antioxidants, mitoTEMPOL and MitoQ, were effective in rodents at preventing cardiac injury while maintaining the anti-tumor efficacy of doxorubicin [285]. By understanding the mechanism of the adverse drug outcome, a targeted adjuvant therapy can prevent cardiotoxicity while not interfering with the anti-cancer efficacy of doxorubicin.

\subsection{Conclusions}

Fe-S clusters are multi-functional protein prosthetic groups highly conserved between bacteria and eukaryotes. In the mitochondria, Fe-S clusters play essential roles in the enzyme aconitase, while also being integral to electron transfer within the respiratory complexes. During mitochondrial respiration, electron transfer through these clusters is associated with ROS production at several distinct sites with Complexes I, II and III. These ROS act as cell signaling molecules, acting within oxygen-sensitive tissues to mediate changes in vascular tone and respiration. Cluster N2 of Complex I has been identified as a potential redox-sensitive oxygen sensor, regulating the production of ROS to initiate cell-signaling cascades within the pulmonary artery and carotid body. Fe-S containing proteins are also suggested to be transcriptionallyregulated mediators in fetal vascular development and are emerging as therapeutic targets in the treatment of diabetes, malaria, and cancer. Numerous questions regarding the role of Fe-S centers in health and disease still remain. For example, it is not known if there is a biochemical threshold for Complex I substrates (i.e., NADH) in oxygen-sensing tissues. Dunham-Snary et al. demonstrated that PASMC exposed to chronic hypoxia (to mimic group 3 pulmonary hypertension) lowered expression of NDUFS2, reduced Complex I activity, and increased the ratio of $\mathrm{NADH} / \mathrm{NAD}^{+}[77]$. Further investigation is required to determine if this relationship is bidirectional, and if NADH deprivation would inhibit oxygen-sensing in PASMC. Further 
investigation is also warranted to determine the effect of mitochondrial dysfunction due to mitochondrial disease on oxygen-sensing. Jain et al. reported that chronic hypoxia exposure activates the HIF signalling cascade, resulting in improved survival and reduced disease severity in a mouse model of Leigh syndrome, one of the most common mitochondrial diseases [236]. However, it remains unknown whether impaired acute oxygen-sensing is linked to mitochondrial diseases, and what HOSS tissues (if any) are affected. With the central role of Fe-S clusters in mitochondria, future investigations into potential links between mitochondrial diseases, acute oxygen-sensing, and Fe-S assembly/function/dysfunction could reveal new therapeutic avenues. 


\section{References}

[1] K.J. Dunham-Snary, Z.G. Hong, P.Y. Xiong, J.C. Del Paggio, J.E. Herr, A.M. Johri, S.L. Archer, A mitochondrial redox oxygen sensor in the pulmonary vasculature and ductus arteriosus, Pflugers Arch 468(1) (2016) 43-58.

[2] R.I. Clyman, Ductus arteriosus: current theories of prenatal and postnatal regulation, Semin Perinatol 11(1) (1987) 64-71.

[3] M.R. Drayton, R. Skidmore, Ductus arteriosus blood flow during first 48 hours of life, Archives of Disease in Childhood 62(10) (1987) 1030-1034.

[4] S.Y. Ho, R.H. Anderson, Anatomical closure of the ductus arteriosus: a study in 35 specimens, J Anat 128(Pt 4) (1979) 829-836.

[5] M. El Hajjar, G. Vaksmann, T. Rakza, G. Kongolo, L. Storme, Severity of the ductal shunt: a comparison of different markers, Arch Dis Child Fetal Neonatal Ed 90(5) (2005) F419-22.

[6] J.E. Dice, J. Bhatia, Patent ductus arteriosus: an overview, J Pediatr Pharmacol Ther 12(3) (2007) 138146.

[7] S.E.G. Hamrick, G. Hansmann, Patent Ductus Arteriosus of the Preterm Infant, Pediatrics 125(5) (2010) 1020-1030.

[8] E.D. Michelakis, I. Rebeyka, X. Wu, A. Nsair, B. Thebaud, K. Hashimoto, J.R. Dyck, A. Haromy, G. Harry, A. Barr, S.L. Archer, O2 sensing in the human ductus arteriosus: regulation of voltage-gated K+ channels in smooth muscle cells by a mitochondrial redox sensor, Circ Res 91(6) (2002) 478-86.

[9] B. Thébaud, E.D. Michelakis, X.-C. Wu, R. Moudgil, M. Kuzyk, J.R. Dyck, G. Harry, K. Hashimoto, A. Haromy, I. Rebeyka, Oxygen-sensitive Kv channel gene transfer confers oxygen responsiveness to preterm rabbit and remodeled human ductus arteriosus: implications for infants with patent ductus arteriosus, Circulation 110(11) (2004) 1372-1379.

[10] T. Takizawa, T. Oda, K. Arishima, M. Yamamoto, T. Masaoka, H. Somiya, F. Akahori, K. Shiota, A calcium channel blocker verapamil inhibits the spontaneous closure of the ductus arteriosus in newborn rats, J Toxicol Sci 19(3) (1994) 171-4.

[11] A.L. Orr, L. Vargas, C.N. Turk, J.E. Baaten, J.T. Matzen, V.J. Dardov, S.J. Attle, J. Li, D.C. Quackenbush, R.L.S. Goncalves, I.V. Perevoshchikova, H.M. Petrassi, S.L. Meeusen, E.K. Ainscow, M.D. Brand, Suppressors of superoxide production from mitochondrial complex III, Nat Chem Biol 11(11) (2015) 834-836.

[12] M.D. Brand, R.L.S. Goncalves, A.L. Orr, L. Vargas, A.A. Gerencser, M. Borch Jensen, Y.T. Wang, S. Melov, C.N. Turk, J.T. Matzen, V.J. Dardov, H.M. Petrassi, S.L. Meeusen, I.V. Perevoshchikova, H. Jasper, P.S. Brookes, E.K. Ainscow, Suppressors of Superoxide- $\mathrm{H}(2) \mathrm{O}(2)$ Production at Site I(Q) of Mitochondrial Complex I Protect against Stem Cell Hyperplasia and Ischemia-Reperfusion Injury, Cell metabolism 24(4) (2016) 582-592.

[13] F. Coceani, C. Armstrong, L. Kelsey, Endothelin is a potent constrictor of the lamb ductus arteriosus, Can J Physiol Pharmacol 67(8) (1989) 902-4.

[14] E.B. Sideris, K. Yokochi, F. Coceani, P.M. Olley, Prostaglandins and fetal cardiac output distribution in the lamb, Am J Physiol 248(6 Pt 2) (1985) H853-8.

[15] F. Coceani, L. Kelsey, E. Seidlitz, K. Korzekwa, Inhibition of the contraction of the ductus arteriosus to oxygen by 1 -aminobenzotriazole, a mechanism-based inactivator of cytochrome $\mathrm{P} 450, \mathrm{Br} \mathrm{J}$ Pharmacol 117(7) (1996) 1586-92.

[16] F. Coceani, L. Kelsey, E. Seidlitz, Carbon monoxide-induced relaxation of the ductus arteriosus in the lamb: evidence against the prime role of guanylyl cyclase, Br J Pharmacol 118(7) (1996) 1689-96.

[17] J.R. Fineman, Y. Takahashi, C. Roman, R.I. Clyman, Endothelin-receptor blockade does not alter closure of the ductus arteriosus, American Journal of Physiology-Heart and Circulatory Physiology 275(5) (1998) H1620-H1626. 
[18] F. Coceani, P.M. Olley, The control of cardiovascular shunts in the fetal and perinatal period, Can J Physiol Pharmacol 66(8) (1988) 1129-34.

[19] F. Coceani, E. Bodach, E. White, I. Bishai, P.M. Olley, Prostaglandin I2 is less relaxant than prostaglandin E2 on the lamb ductus arteriosus, Prostaglandins 15(4) (1978) 551-6.

[20] F. Coceani, D. Huhtanen, N.C. Hamilton, I. Bishai, P.M. Olley, Involvement of intramural prostaglandin E2 in prenatal patency of the lamb ductus arteriosus, Can J Physiol Pharmacol 64(6) (1986) 737-44.

[21] M.A. Heymann, A.M. Rudolph, Effects of acetylsalicylic acid on the ductus arteriosus and circulation in fetal lambs in utero, Circ Res 38(5) (1976) 418-22.

[22] J.M. Rennie, R.W. Cooke, Prolonged low dose indomethacin for persistent ductus arteriosus of prematurity, Archives of Disease in Childhood 66(1 Spec No) (1991) 55-58.

[23] B. Van Overmeire, K. Smets, D. Lecoutere, H. Van de Broek, J. Weyler, K. De Groote, J.-P. Langhendries, A Comparison of Ibuprofen and Indomethacin for Closure of Patent Ductus Arteriosus, New England Journal of Medicine 343(10) (2000) 674-681.

[24] M.A. Heymann, A.M. Rudolph, N.H. Silverman, Closure of the ductus arteriosus in premature infants by inhibition of prostaglandin synthesis, N Engl J Med 295(10) (1976) 530-3.

[25] N. Nakwan, S. Jain, K. Kumar, S. Hosono, M. Hammoud, Y.Y. Elsayed, S. Ariff, B. Hasan, W. Khowaja, W.B. Poon, An Asian multicenter retrospective study on persistent pulmonary hypertension of the newborn: incidence, etiology, diagnosis, treatment and outcome, J Matern Fetal Neonatal Med 33(12) (2020) 2032-2037.

[26] S. Prescott, J. Keim-Malpass, Patent Ductus Arteriosus in the Preterm Infant: Diagnostic and Treatment Options, Advances in Neonatal Care 17(1) (2017) 10-18.

[27] M. Tristani-Firouzi, H.L. Reeve, S. Tolarova, E.K. Weir, S.L. Archer, Oxygen-induced constriction of rabbit ductus arteriosus occurs via inhibition of a 4-aminopyridine-, voltage-sensitive potassium channel, J Clin Invest 98(9) (1996) 1959-65.

[28] E. Michelakis, I. Rebeyka, J. Bateson, P. Olley, L. Puttagunta, S. Archer, Voltage-gated potassium channels in human ductus arteriosus, The Lancet 356(9224) (2000) 134-137.

[29] E.K. Weir, J. López-Barneo, K.J. Buckler, S.L. Archer, Acute oxygen-sensing mechanisms, The New England journal of medicine 353(19) (2005) 2042-2055.

[30] E.K. Weir, S.L. Archer, The role of redox changes in oxygen sensing, Respir Physiol Neurobiol 174(3) (2010) 182-91.

[31] I.F. McMurtry, A.B. Davidson, J.T. Reeves, R.F. Grover, Inhibition of hypoxic pulmonary vasoconstriction by calcium antagonists in isolated rat lungs, Circ Res 38(2) (1976) 99-104.

[32] D.R. Harder, J.A. Madden, C. Dawson, Hypoxic induction of Ca2+-dependent action potentials in small pulmonary arteries of the cat, J Appl Physiol (1985) 59(5) (1985) 1389-93.

[33] K. Hasunuma, D.M. Rodman, I.F. McMurtry, Effects of K+ channel blockers on vascular tone in the perfused rat lung, Am Rev Respir Dis 144(4) (1991) 884-7.

[34] S.M. Tipparaju, X.-P. Li, P.J. Kilfoil, B. Xue, V.N. Uversky, A. Bhatnagar, O.A. Barski, Interactions between the $\mathrm{C}$-terminus of $\mathrm{Kv} 1.5$ and $\mathrm{Kv} \beta$ regulate pyridine nucleotide-dependent changes in channel gating, Pflugers Archiv : European journal of physiology 463(6) (2012) 799-818.

[35] B. Thebaud, E.D. Michelakis, X.C. Wu, R. Moudgil, M. Kuzyk, J.R. Dyck, G. Harry, K. Hashimoto, A. Haromy, I. Rebeyka, S.L. Archer, Oxygen-sensitive Kv channel gene transfer confers oxygen responsiveness to preterm rabbit and remodeled human ductus arteriosus: implications for infants with patent ductus arteriosus, Circulation 110(11) (2004) 1372-9.

[36] S.L. Archer, E. Souil, A.T. Dinh-Xuan, B. Schremmer, J.C. Mercier, A. El Yaagoubi, L. NguyenHuu, H.L. Reeve, V. Hampl, Molecular identification of the role of voltage-gated K+ channels, Kv1.5 and Kv2.1, in hypoxic pulmonary vasoconstriction and control of resting membrane potential in rat pulmonary artery myocytes, J Clin Invest 101(11) (1998) 2319-30.

[37] M.K. Park, Y.M. Bae, S.H. Lee, W.K. Ho, Y.E. Earm, Modulation of voltage-dependent K+ channel by redox potential in pulmonary and ear arterial smooth muscle cells of the rabbit, Pflugers Arch 434(6) (1997) 764-71. 
[38] J.P. Ruppersberg, M. Stocker, O. Pongs, S.H. Heinemann, R. Frank, M. Koenen, Regulation of fast inactivation of cloned mammalian IK(A) channels by cysteine oxidation, Nature 352(6337) (1991) 711-4. [39] J.T. Hulme, E.A. Coppock, A. Felipe, J.R. Martens, M.M. Tamkun, Oxygen sensitivity of cloned voltage-gated $\mathrm{K}(+)$ channels expressed in the pulmonary vasculature, Circ Res 85(6) (1999) 489-97. [40] Z. Hong, S. Kutty, P.T. Toth, G. Marsboom, J.M. Hammel, C. Chamberlain, J.J. Ryan, H.J. Zhang, W.W. Sharp, E. Morrow, K. Trivedi, E.K. Weir, S.L. Archer, Role of dynamin-related protein 1 (Drp1)mediated mitochondrial fission in oxygen sensing and constriction of the ductus arteriosus, Circ Res 112(5) (2013) 802-15.

[41] M.T. Breitzig, M.D. Alleyn, R.F. Lockey, N. Kolliputi, A mitochondrial delicacy: dynamin-related protein 1 and mitochondrial dynamics, Am J Physiol Cell Physiol 315(1) (2018) C80-C90.

[42] A. Cassidy-Stone, J.E. Chipuk, E. Ingerman, C. Song, C. Yoo, T. Kuwana, M.J. Kurth, J.T. Shaw, J.E. Hinshaw, D.R. Green, J. Nunnari, Chemical inhibition of the mitochondrial division dynamin reveals its role in Bax/Bak-dependent mitochondrial outer membrane permeabilization, Dev Cell 14(2) (2008) 193-204.

[43] H.L. Reeve, E.K. Weir, D.P. Nelson, D.A. Peterson, S.L. Archer, Opposing effects of oxidants and antioxidants on $\mathrm{K}+$ channel activity and tone in rat vascular tissue, Exp Physiol 80(5) (1995) 825-34.

[44] J. Rettig, S.H. Heinemann, F. Wunder, C. Lorra, D.N. Parcej, J. Oliver Dolly, O. Pongs, Inactivation properties of voltage-gated $\mathrm{K}+$ channels altered by presence of $\beta$-subunit, Nature 369(6478) (1994) 289294.

[45] A. Olschewski, Z. Hong, D.A. Peterson, D.P. Nelson, V.A. Porter, E.K. Weir, Opposite effects of redox status on membrane potential, cytosolic calcium, and tone in pulmonary arteries and ductus arteriosus, American Journal of Physiology-Lung Cellular and Molecular Physiology 286(1) (2004) L15L22.

[46] H.-S. Wong, P.A. Dighe, V. Mezera, P.-A. Monternier, M.D. Brand, Production of superoxide and hydrogen peroxide from specific mitochondrial sites under different bioenergetic conditions, The Journal of biological chemistry 292(41) (2017) 16804-16809.

[47] J.F. Turrens, Mitochondrial formation of reactive oxygen species, J Physiol 552(Pt 2) (2003) 335-44.

[48] E. Cadenas, K.J. Davies, Mitochondrial free radical generation, oxidative stress, and aging, Free Radic Biol Med 29(3-4) (2000) 222-30.

[49] J.F. Turrens, A. Boveris, Generation of superoxide anion by the NADH dehydrogenase of bovine heart mitochondria, Biochem J 191(2) (1980) 421-427.

[50] K. Sugioka, M. Nakano, H. Totsune-Nakano, H. Minakami, S. Tero-Kubota, Y. Ikegami, Mechanism of O2- generation in reduction and oxidation cycle of ubiquinones in a model of mitochondrial electron transport systems, Biochim Biophys Acta 936(3) (1988) 377-85.

[51] C.L. Quinlan, A.L. Orr, I.V. Perevoshchikova, J.R. Treberg, B.A. Ackrell, M.D. Brand, Mitochondrial complex II can generate reactive oxygen species at high rates in both the forward and reverse reactions, J Biol Chem 287(32) (2012) 27255-64.

[52] S. Kaewpila, S. Venkataraman, G.R. Buettner, L.W. Oberley, Manganese superoxide dismutase modulates hypoxia-inducible factor-1 alpha induction via superoxide, Cancer Res 68(8) (2008) 2781-8.

[53] J.R. Burgoyne, S.-i. Oka, N. Ale-Agha, P. Eaton, Hydrogen peroxide sensing and signaling by protein kinases in the cardiovascular system, Antioxid Redox Signal 18(9) (2013) 1042-1052.

[54] S.L. Archer, H.L. Reeve, E. Michelakis, L. Puttagunta, R. Waite, D.P. Nelson, M.C. Dinauer, E.K. Weir, O2 sensing is preserved in mice lacking the gp91 phox subunit of NADPH oxidase, Proc Natl Acad Sci U S A 96(14) (1999) 7944-9.

[55] R.D. Jones, J.T. Hancock, A.H. Morice, NADPH oxidase: a universal oxygen sensor?, Free Radic Biol Med 29(5) (2000) 416-24.

[56] B.A. Freeman, J.D. Crapo, Hyperoxia increases oxygen radical production in rat lungs and lung mitochondria, J Biol Chem 256(21) (1981) 10986-92.

[57] B.A. Freeman, M.K. Topolosky, J.D. Crapo, Hyperoxia increases oxygen radical production in rat lung homogenates, Arch Biochem Biophys 216(2) (1982) 477-84. 
[58] N. Oshino, D. Jamieson, B. Chance, The properties of hydrogen peroxide production under hyperoxic and hypoxic conditions of perfused rat liver, Biochem J 146(1) (1975) 53-65.

[59] S.K. Berkelhamer, G.A. Kim, J.E. Radder, S. Wedgwood, L. Czech, R.H. Steinhorn, P.T.

Schumacker, Developmental differences in hyperoxia-induced oxidative stress and cellular responses in the murine lung, Free Radic Biol Med 61 (2013) 51-60.

[60] E.D. Michelakis, S.L. Archer, E.K. Weir, Acute hypoxic pulmonary vasoconstriction: a model of oxygen sensing, Physiol Res 44(6) (1995) 361-7.

[61] S.L. Archer, D.P. Nelson, E.K. Weir, Simultaneous measurement of O2 radicals and pulmonary vascular reactivity in rat lung, J Appl Physiol (1985) 67(5) (1989) 1903-11.

[62] J. Duranteau, N.S. Chandel, A. Kulisz, Z. Shao, P.T. Schumacker, Intracellular signaling by reactive oxygen species during hypoxia in cardiomyocytes, J Biol Chem 273(19) (1998) 11619-24.

[63] T.L. Vanden Hoek, L.B. Becker, Z. Shao, C. Li, P.T. Schumacker, Reactive oxygen species released from mitochondria during brief hypoxia induce preconditioning in cardiomyocytes, J Biol Chem 273(29) (1998) 18092-8.

[64] N.S. Chandel, W.C. Trzyna, D.S. McClintock, P.T. Schumacker, Role of oxidants in NF-kappa B activation and TNF-alpha gene transcription induced by hypoxia and endotoxin, J Immunol 165(2) (2000) 1013-21.

[65] G.B. Waypa, J.D. Marks, M.M. Mack, C. Boriboun, P.T. Mungai, P.T. Schumacker, Mitochondrial reactive oxygen species trigger calcium increases during hypoxia in pulmonary arterial myocytes, Circ Res 91(8) (2002) 719-26.

[66] R.D. Guzy, B. Hoyos, E. Robin, H. Chen, L. Liu, K.D. Mansfield, M.C. Simon, U. Hammerling, P.T. Schumacker, Mitochondrial complex III is required for hypoxia-induced ROS production and cellular oxygen sensing, Cell Metab 1(6) (2005) 401-8.

[67] L. Gao, P. González-Rodríguez, P. Ortega-Sáenz, J. López-Barneo, Redox signaling in acute oxygen sensing, Redox Biol 12 (2017) 908-915.

[68] N. Sommer, M. Hüttemann, O. Pak, S. Scheibe, F. Knoepp, C. Sinkler, M. Malczyk, M. Gierhardt, A. Esfandiary, S. Kraut, F. Jonas, C. Veith, S. Aras, A. Sydykov, N. Alebrahimdehkordi, K. Giehl, M. Hecker, R.P. Brandes, W. Seeger, F. Grimminger, H.A. Ghofrani, R.T. Schermuly, L.I. Grossman, N. Weissmann, Mitochondrial Complex IV Subunit 4 Isoform 2 Is Essential for Acute Pulmonary Oxygen Sensing, Circ Res 121(4) (2017) 424-438.

[69] I. Arias-Mayenco, P. Gonzalez-Rodriguez, H. Torres-Torrelo, L. Gao, M.C. Fernandez-Aguera, V. Bonilla-Henao, P. Ortega-Saenz, J. Lopez-Barneo, Acute O2 Sensing: Role of Coenzyme QH2/Q Ratio and Mitochondrial ROS Compartmentalization, Cell Metab 28(1) (2018) 145-158 e4.

[70] T.D. Henry, S.L. Archer, D. Nelson, E.K. Weir, A.H. From, Enhanced chemiluminescence as a measure of oxygen-derived free radical generation during ischemia and reperfusion, Circ Res 67(6) (1990) 1453-61.

[71] H.L. Reeve, S. Tolarova, D.P. Nelson, S. Archer, E.K. Weir, Redox control of oxygen sensing in the rabbit ductus arteriosus, J Physiol 533(Pt 1) (2001) 253-61.

[72] S.L. Archer, D.P. Nelson, E.K. Weir, Detection of activated $\mathrm{O} 2$ species in vitro and in rat lungs by chemiluminescence, J Appl Physiol (1985) 67(5) (1989) 1912-21.

[73] S.L. Archer, J. Huang, T. Henry, D. Peterson, E.K. Weir, A redox-based O2 sensor in rat pulmonary vasculature, Circ Res 73(6) (1993) 1100-12.

[74] S.L. Archer, J.A. Will, E.K. Weir, Redox status in the control of pulmonary vascular tone, Herz 11(3) (1986) 127-41.

[75] P. Ortega-Saenz, R. Pardal, M. Garcia-Fernandez, J. Lopez-Barneo, Rotenone selectively occludes sensitivity to hypoxia in rat carotid body glomus cells, J Physiol 548(Pt 3) (2003) 789-800.

[76] S. Bonnet, E.D. Michelakis, C.J. Porter, M.A. Andrade-Navarro, B. Thebaud, S. Bonnet, A. Haromy, G. Harry, R. Moudgil, M.S. McMurtry, E.K. Weir, S.L. Archer, An abnormal mitochondrial-hypoxia inducible factor-1alpha-Kv channel pathway disrupts oxygen sensing and triggers pulmonary arterial hypertension in fawn hooded rats: similarities to human pulmonary arterial hypertension, Circulation 113(22) (2006) 2630-41. 
[77] K.J. Dunham-Snary, D. Wu, F. Potus, E.A. Sykes, J.D. Mewburn, R.L. Charles, P. Eaton, R.A. Sultanian, S.L. Archer, Ndufs2, a Core Subunit of Mitochondrial Complex I, Is Essential for Acute Oxygen-Sensing and Hypoxic Pulmonary Vasoconstriction, Circ Res 124(12) (2019) 1727-1746.

[78] M.C. Fernández-Agüera, L. Gao, P. González-Rodríguez, C.O. Pintado, I. Arias-Mayenco, P. García-Flores, A. García-Pergañeda, A. Pascual, P. Ortega-Sáenz, J. López-Barneo, Oxygen Sensing by Arterial Chemoreceptors Depends on Mitochondrial Complex I Signaling, Cell Metab 22(5) (2015) 82537.

[79] G.B. Waypa, J.D. Marks, R.D. Guzy, P.T. Mungai, J.M. Schriewer, D. Dokic, M.K. Ball, P.T. Schumacker, Superoxide generated at mitochondrial complex III triggers acute responses to hypoxia in the pulmonary circulation, Am J Respir Crit Care Med 187(4) (2013) 424-32.

[80] J.F. Turrens, A. Alexandre, A.L. Lehninger, Ubisemiquinone is the electron donor for superoxide formation by complex III of heart mitochondria, Arch Biochem Biophys 237(2) (1985) 408-14.

[81] L. Conte, V. Zara, The Rieske Iron-Sulfur Protein: Import and Assembly into the Cytochrome bc(1) Complex of Yeast Mitochondria, Bioinorg Chem Appl 2011 (2011) 363941-363941.

[82] G.B. Waypa, N.S. Chandel, P.T. Schumacker, Model for hypoxic pulmonary vasoconstriction involving mitochondrial oxygen sensing, Circ Res 88(12) (2001) 1259-66.

[83] H. Kajimoto, K. Hashimoto, S.N. Bonnet, A. Haromy, G. Harry, R. Moudgil, T. Nakanishi, I. Rebeyka, B. Thebaud, E.D. Michelakis, S.L. Archer, Oxygen activates the Rho/Rho-kinase pathway and induces RhoB and ROCK-1 expression in human and rabbit ductus arteriosus by increasing mitochondriaderived reactive oxygen species: a newly recognized mechanism for sustaining ductal constriction, Circulation 115(13) (2007) 1777-88.

[84] A.P. Somlyo, A.V. Somlyo, Ca2+ sensitivity of smooth muscle and nonmuscle myosin II: modulated by G proteins, kinases, and myosin phosphatase, Physiol Rev 83(4) (2003) 1325-58.

[85] R.L. Goncalves, C.L. Quinlan, I.V. Perevoshchikova, M. Hey-Mogensen, M.D. Brand, Sites of superoxide and hydrogen peroxide production by muscle mitochondria assessed ex vivo under conditions mimicking rest and exercise, J Biol Chem 290(1) (2015) 209-27.

[86] M.D. Brand, Mitochondrial generation of superoxide and hydrogen peroxide as the source of mitochondrial redox signaling, Free Radical Biology and Medicine 100 (2016) 14-31.

[87] N. Xiong, X. Long, J. Xiong, M. Jia, C. Chen, J. Huang, D. Ghoorah, X. Kong, Z. Lin, T. Wang, Mitochondrial complex I inhibitor rotenone-induced toxicity and its potential mechanisms in Parkinson's disease models, Crit Rev Toxicol 42(7) (2012) 613-32.

[88] L. Piao, Y.H. Fang, R.B. Hamanaka, G.M. Mutlu, C. Dezfulian, S.L. Archer, W.W. Sharp, Suppression of Superoxide-Hydrogen Peroxide Production at Site IQ of Mitochondrial Complex I Attenuates Myocardial Stunning and Improves Postcardiac Arrest Outcomes, Crit Care Med 48(2) (2020) e133-e140.

[89] M. Cucerea, M. Simon, E. Moldovan, M. Ungureanu, R. Marian, L. Suciu, Congenital Heart Disease Requiring Maintenance of Ductus Arteriosus in Critically Ill Newborns Admitted at a Tertiary Neonatal Intensive Care Unit, J Crit Care Med (Targu Mures) 2(4) (2016) 185-191.

[90] E.K. Weir, J. López-Barneo, K.J. Buckler, S.L. Archer, Acute oxygen-sensing mechanisms, N Engl J Med 353(19) (2005) 2042-55.

[91] A.L. Orr, L. Vargas, C.N. Turk, J.E. Baaten, J.T. Matzen, V.J. Dardov, S.J. Attle, J. Li, D.C. Quackenbush, R.L. Goncalves, I.V. Perevoshchikova, H.M. Petrassi, S.L. Meeusen, E.K. Ainscow, M.D. Brand, Suppressors of superoxide production from mitochondrial complex III, Nat Chem Biol 11(11) (2015) 834-6.

[92] S. Burén, E. Jiménez-Vicente, C. Echavarri-Erasun, L.M. Rubio, Biosynthesis of Nitrogenase Cofactors, Chem Rev 120(12) (2020) 4921-4968.

[93] Y. Luo, C.E. Ergenekan, J.T. Fischer, M.L. Tan, T. Ichiye, The molecular determinants of the increased reduction potential of the rubredoxin domain of rubrerythrin relative to rubredoxin, Biophys $\mathrm{J}$ 98(4) (2010) 560-8.

[94] K.M. Ewen, M. Ringle, R. Bernhardt, Adrenodoxin--a versatile ferredoxin, IUBMB Life 64(6) (2012) 506-12. 
[95] M.E. Ali, N.N. Nair, M. Retegan, F. Neese, V. Staemmler, D. Marx, The iron-sulfur core in Rieske proteins is not symmetric, JBIC Journal of Biological Inorganic Chemistry 19(8) (2014) 1287-1293.

[96] T. Ohnishi, Iron-sulfur clusters/semiquinones in Complex I, Biochimica et Biophysica Acta (BBA) Bioenergetics 1364(2) (1998) 186-206.

[97] V. Yankovskaya, R. Horsefield, S. Törnroth, C. Luna-Chavez, H. Miyoshi, C. Léger, B. Byrne, G. Cecchini, S. Iwata, Architecture of Succinate Dehydrogenase and Reactive Oxygen Species Generation, Science 299(5607) (2003) 700.

[98] A.H. Robbins, C.D. Stout, The structure of aconitase, Proteins 5(4) (1989) 289-312.

[99] N.D. Lanz, S.J. Booker, Auxiliary iron-sulfur cofactors in radical SAM enzymes, Biochim Biophys Acta 1853(6) (2015) 1316-34.

[100] K. Kobayashi, M. Fujikawa, T. Kozawa, Oxidative stress sensing by the iron-sulfur cluster in the transcription factor, SoxR, J Inorg Biochem 133 (2014) 87-91.

[101] P.J. Kiley, H. Beinert, Oxygen sensing by the global regulator, FNR: the role of the iron-sulfur cluster, FEMS Microbiol Rev 22(5) (1998) 341-52.

[102] M.W. Hentze, L.C. Kühn, Molecular control of vertebrate iron metabolism: mRNA-based regulatory circuits operated by iron, nitric oxide, and oxidative stress, Proc Natl Acad Sci U S A 93(16) (1996) 8175-8182.

[103] M.F. White, Structure, function and evolution of the XPD family of iron-sulfur-containing 5'-->3' DNA helicases, Biochem Soc Trans 37(Pt 3) (2009) 547-51.

[104] J. Stiban, G.A. Farnum, S.L. Hovde, L.S. Kaguni, The N-terminal domain of the Drosophila mitochondrial replicative DNA helicase contains an iron-sulfur cluster and binds DNA, J Biol Chem 289(35) (2014) 24032-42.

[105] L. Mariotti, S. Wild, G. Brunoldi, A. Piceni, I. Ceppi, S. Kummer, R.E. Lutz, P. Cejka, K. Gari, The iron-sulphur cluster in human DNA2 is required for all biochemical activities of DNA2, Commun Biol 3(1) (2020) 322 .

[106] M.F. White, M.S. Dillingham, Iron-sulphur clusters in nucleic acid processing enzymes, Current Opinion in Structural Biology 22(1) (2012) 94-100.

[107] A.G. Baranovskiy, H.M. Siebler, Y.I. Pavlov, T.H. Tahirov, Iron-Sulfur Clusters in DNA

Polymerases and Primases of Eukaryotes, Methods Enzymol 599 (2018) 1-20.

[108] K. Saikrishnan, J.T. Yeeles, N.S. Gilhooly, W.W. Krajewski, M.S. Dillingham, D.B. Wigley, Insights into Chi recognition from the structure of an AddAB-type helicase-nuclease complex, Embo $\mathrm{j}$ 31(6) (2012) 1568-78.

[109] R. Altmann, Die Elementarorganismen und ihre Beziehungen zu den Zellen, Veit1894.

[110] A. Kölliker, Zur Kenntnis der quergestreiften Muskelfasern, Wilh. Engelmann1888.

[111] I.E. Wallin, Symbionticism and the origin of species, Williams \& Wilkins Company, Baltimore, 1927.

[112] L. Sagan, On the origin of mitosing cells, J Theor Biol 14(3) (1967) 255-74.

[113] L. Margulis, Origin of eukaryotic cells: Evidence and research implications for a theory of the origin and evolution of microbial, plant and animal cells on the precambrian Earth, Yale University Press 1970.

[114] L. Margulis, Symbiosis in cell evolution: Life and its environment on the early earth, (1981).

[115] J. Lederberg, Cell genetics and hereditary symbiosis, Physiol Rev 32(4) (1952) 403-30.

[116] H. Ris, W. Plaut, Ultrastructure of DNA-containing areas in the chloroplast of Chlamydomonas, J Cell Biol 13 (1962) 383-91.

[117] E.B. Wilson, The cell in development and heredity, Macmillan1925.

[118] J.B.S. Haldane, The Origins of Life, in: M.L. Johnson, M. Abercrombie, G.E. Fogg (Eds.), New Biology, No. 16, Penguin Books, London, 1954.

[119] M.W. Gray, W.F. Doolittle, Has the endosymbiont hypothesis been proven?, Microbiological Reviews 46(1) (1982) 1-42.

[120] T. Cavalier-Smith, The origin of nuclei and of eukaryotic cells, Nature 256(5517) (1975) 463-468. 
[121] E.O. Dodson, Crossing the prokaryote-eucaryote border: endosymbiosis or continuous development?, Canadian journal of microbiology 25(6) (1979) 651-674.

[122] W.F. Doolittle, Evolutionary molecular biology: where is it going?, Canadian Journal of Biochemistry 60(2) (1982) 83-90.

[123] F. Taylor, Autogenous theories for the origin of eukaryotes, Taxon (1976) 377-390.

[124] D.A. Baum, A comparison of autogenous theories for the origin of eukaryotic cells, American journal of botany 102(12) (2015) 1954-1965.

[125] G. Jékely, Origin of eukaryotic endomembranes: a critical evaluation of different model scenarios, Eukaryotic Membranes and Cytoskeleton (2007) 38-51.

[126] D. Yang, Y. Oyaizu, H. Oyaizu, G.J. Olsen, C.R. Woese, Mitochondrial origins, Proc Natl Acad Sci U S A 82(13) (1985) 4443-7.

[127] Z. Wang, M. Wu, An integrated phylogenomic approach toward pinpointing the origin of mitochondria, Sci Rep 5 (2015) 7949.

[128] M.W. Gray, D.F. Spencer, Evolution of microbial life, Cambridge University Press 1996.

[129] D.A. Fitzpatrick, C.J. Creevey, J.O. McInerney, Genome phylogenies indicate a meaningful alphaproteobacterial phylogeny and support a grouping of the mitochondria with the Rickettsiales, Mol Biol Evol 23(1) (2006) 74-85.

[130] A.J. Roger, S.A. Muñoz-Gómez, R. Kamikawa, The origin and diversification of mitochondria, Current Biology 27(21) (2017) R1177-R1192.

[131] C.D. Dunn, Some Liked It Hot: A Hypothesis Regarding Establishment of the Proto-Mitochondrial Endosymbiont During Eukaryogenesis, J Mol Evol 85(3-4) (2017) 99-106.

[132] J.M. Whatley, P. John, F.R. Whatley, From extracellular to intracellular: the establishment of mitochondria and chloroplasts, Proc R Soc Lond B Biol Sci 204(1155) (1979) 165-87.

[133] D. Speijer, Alternating terminal electron-acceptors at the basis of symbiogenesis: How oxygen ignited eukaryotic evolution, Bioessays 39(2) (2017).

[134] S.G. Andersson, C.G. Kurland, Origins of mitochondria and hydrogenosomes, Curr Opin Microbiol 2(5) (1999) 535-41.

[135] A.D. Tsaousis, On the origin of iron/sulfur cluster biosynthesis in eukaryotes, Frontiers in microbiology 10 (2019) 2478.

[136] R. Lill, K. Diekert, A. Kaut, H. Lange, W. Pelzer, C. Prohl, G. Kispal, The essential role of mitochondria in the biogenesis of cellular iron-sulfur proteins, (1999).

[137] J.J. Braymer, R. Lill, Iron-sulfur cluster biogenesis and trafficking in mitochondria, Journal of Biological Chemistry 292(31) (2017) 12754-12763.

[138] T.A. Rouault, N. Maio, Biogenesis and functions of mammalian iron-sulfur proteins in the regulation of iron homeostasis and pivotal metabolic pathways, Journal of Biological Chemistry 292(31) (2017) 12744-12753.

[139] P. Peña-Diaz, J. Lukeš, Fe-S cluster assembly in the supergroup Excavata, JBIC Journal of Biological Inorganic Chemistry 23(4) (2018) 521-541.

[140] Y. Davidov, D. Huchon, S.F. Koval, E. Jurkevitch, A new alpha-proteobacterial clade of Bdellovibrio-like predators: implications for the mitochondrial endosymbiotic theory, Environ Microbiol 8(12) (2006) 2179-88.

[141] Y. Davidov, E. Jurkevitch, Predation between prokaryotes and the origin of eukaryotes, Bioessays 31(7) (2009) 748-57.

[142] J.E. Walker, The NADH:ubiquinone oxidoreductase (complex I) of respiratory chains, Q Rev Biophys 25(3) (1992) 253-324.

[143] T. Yagi, A. Matsuno-Yagi, The proton-translocating NADH-quinone oxidoreductase in the respiratory chain: the secret unlocked, Biochemistry 42(8) (2003) 2266-74.

[144] G. Burger, B.F. Lang, M. Reith, M.W. Gray, Genes encoding the same three subunits of respiratory complex II are present in the mitochondrial DNA of two phylogenetically distant eukaryotes, Proc Natl Acad Sci U S A 93(6) (1996) 2328-32. 
[145] C. Hunte, J. Koepke, C. Lange, T. Rossmanith, H. Michel, Structure at 2.3 A resolution of the cytochrome bc(1) complex from the yeast Saccharomyces cerevisiae co-crystallized with an antibody Fv fragment, Structure 8(6) (2000) 669-84.

[146] Z. Zhang, L. Huang, V.M. Shulmeister, Y.I. Chi, K.K. Kim, L.W. Hung, A.R. Crofts, E.A. Berry, S.H. Kim, Electron transfer by domain movement in cytochrome bc1, Nature 392(6677) (1998) 677-84. [147] D.S. Friend, The fine structure of Giardia muris, The Journal of cell biology 29(2) (1966) 317-332. [148] R.M. Rosenbaum, M. Wittner, Ultrastructure of bacterized and axenic trophozoites of Entamoeba histolytica with particular reference to helical bodies, The Journal of cell biology 45(2) (1970) 367-382. [149] M. van der Giezen, Hydrogenosomes and mitosomes: conservation and evolution of functions, $\mathrm{J}$ Eukaryot Microbiol 56(3) (2009) 221-31.

[150] T. Friedrich, K. Steinmuller, H. Weiss, The proton-pumping respiratory complex I of bacteria and mitochondria and its homologue in chloroplasts, FEBS Lett 367(2) (1995) 107-11.

[151] T. Joseph-Horne, D.W. Hollomon, P.M. Wood, Fungal respiration: a fusion of standard and alternative components, Biochim Biophys Acta 1504(2-3) (2001) 179-95.

[152] T. Yagi, Bacterial NADH-quinone oxidoreductases, J Bioenerg Biomembr 23(2) (1991) 211-25.

[153] S. De Vries, R. Van Witzenburg, L.A. Grivell, C.A. Marres, Primary structure and import pathway of the rotenone-insensitive NADH-ubiquinone oxidoreductase of mitochondria from Saccharomyces cerevisiae, Eur J Biochem 203(3) (1992) 587-92.

[154] M.A. Luttik, K.M. Overkamp, P. Kotter, S. de Vries, J.P. van Dijken, J.T. Pronk, The

Saccharomyces cerevisiae NDE1 and NDE2 genes encode separate mitochondrial NADH dehydrogenases catalyzing the oxidation of cytosolic NADH, J Biol Chem 273(38) (1998) 24529-34.

[155] N. Minagawa, A. Yoshimoto, The induction of cyanide-resistant respiration in Hansenula anomala, J Biochem 101(5) (1987) 1141-6.

[156] A.L. Moore, J.N. Siedow, The regulation and nature of the cyanide-resistant alternative oxidase of plant mitochondria, Biochim Biophys Acta 1059(2) (1991) 121-40.

[157] M.S. Albury, P. Dudley, F.Z. Watts, A.L. Moore, Targeting the plant alternative oxidase protein to Schizosaccharomyces pombe mitochondria confers cyanide-insensitive respiration, J Biol Chem 271(29) (1996) 17062-6.

[158] D.G. Nicholls, S.J. Fergusion, Bioenergetics, 4 ed., Academic Press2013.

[159] C.P. Anderson, M. Shen, R.S. Eisenstein, E.A. Leibold, Mammalian iron metabolism and its control by iron regulatory proteins, Biochimica et Biophysica Acta (BBA) - Molecular Cell Research 1823(9) (2012) 1468-1483.

[160] T.A. Rouault, The role of iron regulatory proteins in mammalian iron homeostasis and disease, Nat Chem Biol 2(8) (2006) 406-14.

[161] H. Beinert, M.C. Kennedy, Aconitase, a two-faced protein: enzyme and iron regulatory factor, Faseb j 7(15) (1993) 1442-9.

[162] A. Hausladen, I. Fridovich, Superoxide and peroxynitrite inactivate aconitases, but nitric oxide does not, J Biol Chem 269(47) (1994) 29405-8.

[163] P.R. Gardner, I. Fridovich, Superoxide sensitivity of the Escherichia coli aconitase, J Biol Chem 266(29) (1991) 19328-33.

[164] A.-L. Bulteau, M. Ikeda-Saito, L.I. Szweda, Redox-Dependent Modulation of Aconitase Activity in Intact Mitochondria, Biochemistry 42(50) (2003) 14846-14855.

[165] L. Castro, V. Tórtora, S. Mansilla, R. Radi, Aconitases: Non-redox Iron-Sulfur Proteins Sensitive to Reactive Species, Accounts of Chemical Research 52(9) (2019) 2609-2619.

[166] N. Maio, T.A. Rouault, Outlining the Complex Pathway of Mammalian Fe-S Cluster Biogenesis, Trends Biochem Sci 45(5) (2020) 411-426.

[167] M. Alfadhel, M. Nashabat, Q.A. Ali, K. Hundallah, Mitochondrial iron-sulfur cluster biogenesis from molecular understanding to clinical disease, Neurosciences Journal 22(1) (2017) 4.

[168] K. Leonard, H. Haiker, H. Weiss, Three-dimensional structure of NADH: Ubiquinone reductase (complex I) from Neurospora mitochondria determined by electron microscopy of membrane crystals, Journal of Molecular Biology 194(2) (1987) 277-286. 
[169] G. Hofhaus, H. Weiss, K. Leonard, Electron microscopic analysis of the peripheral and membrane parts of mitochondrial NADH dehydrogenase (Complex I), Journal of Molecular Biology 221(3) (1991) 1027-1043.

[170] V. Guénebaut, R. Vincentelli, D. Mills, H. Weiss, K.R. Leonard, Three-dimensional structure of NADH-dehydrogenase from Neurospora crassa by electron microscopy and conical tilt reconstruction11Edited by K. Nagai, Journal of Molecular Biology 265(4) (1997) 409-418.

[171] R.J.R.J. Janssen, L.G. Nijtmans, L.P.v.d. Heuvel, J.A.M. Smeitink, Mitochondrial complex I: Structure, function and pathology, Journal of Inherited Metabolic Disease 29(4) (2006) 499-515. [172] J. Carroll, R.J. Shannon, I.M. Fearnley, J.E. Walker, J. Hirst, Definition of the Nuclear Encoded Protein Composition of Bovine Heart Mitochondrial Complex I: IDENTIFICATION OF TWO NEW SUBUNITS*, Journal of Biological Chemistry 277(52) (2002) 50311-50317.

[173] C. Ugalde, R. Vogel, R. Huijbens, B. van den Heuvel, J. Smeitink, L. Nijtmans, Human mitochondrial complex I assembles through the combination of evolutionary conserved modules: a framework to interpret complex I deficiencies, Human Molecular Genetics 13(20) (2004) 2461-2472. [174] R.O. Vogel, J.A.M. Smeitink, L.G.J. Nijtmans, Human mitochondrial complex I assembly: A dynamic and versatile process, Biochimica et Biophysica Acta (BBA) - Bioenergetics 1767(10) (2007) 1215-1227.

[175] M. Mimaki, X. Wang, M. McKenzie, D.R. Thorburn, M.T. Ryan, Understanding mitochondrial complex I assembly in health and disease, Biochimica et Biophysica Acta (BBA) - Bioenergetics 1817(6) (2012) 851-862.

[176] T. Friedrich, D.K. Dekovic, S. Burschel, Assembly of the Escherichia coli NADH:ubiquinone oxidoreductase (respiratory complex I), Biochimica et Biophysica Acta (BBA) - Bioenergetics 1857(3) (2016) 214-223.

[177] A. Videira, Complex I from the fungus Neurospora crassa, Biochimica et Biophysica Acta (BBA) Bioenergetics 1364(2) (1998) 89-100.

[178] I. Marques, M. Duarte, J. Assunção, A.V. Ushakova, A. Videira, Composition of complex I from Neurospora crassa and disruption of two "accessory" subunits, Biochimica et Biophysica Acta (BBA) Bioenergetics 1707(2) (2005) 211-220.

[179] U. Brandt, Energy Converting NADH: Quinone Oxidoreductase (Complex I), Annual Review of Biochemistry 75(1) (2006) 69-92.

[180] E. Nakamaru-Ogiso, Iron-Sulfur Clusters in Complex I, in: L. Sazanov (Ed.), A Structural Perspective on Respiratory Complex I: Structure and Function of NADH:ubiquinone oxidoreductase, Springer Netherlands, Dordrecht, 2012, pp. 61-79.

[181] D.-C. Wang, S.W. Meinhardt, U. Sackmann, H. Weiss, T. Ohnishi, The iron-sulfur clusters in the two related forms of mitochondrial NADH: ubiquinone oxidoreductase made by Neurospora crassa, European Journal of Biochemistry 197(1) (1991) 257-264.

[182] T. Rasmussen, D. Scheide, B. Brors, L. Kintscher, H. Weiss, T. Friedrich, Identification of two tetranuclear FeS clusters on the ferredoxin-type subunit of NADH:ubiquinone oxidoreductase (complex I), Biochemistry 40(20) (2001) 6124-31.

[183] L.A. Sazanov, P. Hinchliffe, Structure of the Hydrophilic Domain of Respiratory Complex I from \&lt;em\&gt; Thermus thermophilus\&lt;/em\&gt, Science 311(5766) (2006) 1430.

[184] P.J. Holt, R.G. Efremov, E. Nakamaru-Ogiso, L.A. Sazanov, Reversible FMN dissociation from Escherichia coli respiratory complex I, Biochimica et Biophysica Acta (BBA) - Bioenergetics 1857(11) (2016) 1777-1785.

[185] T. Hayashi, A. Stuchebrukhov, Electron tunneling pathways in respiratory complex I. The role of the internal water between the enzyme subunits, J Electroanal Chem (Lausanne) 660(2) (2011) 356-359. [186] T. Yagi, A. Matsuno-Yagi, The Proton-Translocating NADH-Quinone Oxidoreductase in the Respiratory Chain: The Secret Unlocked, Biochemistry 42(8) (2003) 2266-2274.

[187] V.D. Sled, N.I. Rudnitzky, Y. Hatefi, T. Ohnishi, Thermodynamic analysis of flavin in mitochondrial NADH:ubiquinone oxidoreductase (complex I), Biochemistry 33(33) (1994) 10069-75. 
[188] M. Schulte, K. Frick, E. Gnandt, S. Jurkovic, S. Burschel, R. Labatzke, K. Aierstock, D. Fiegen, D. Wohlwend, S. Gerhardt, O. Einsle, T. Friedrich, A mechanism to prevent production of reactive oxygen species by Escherichia coli respiratory complex I, Nat Commun 10(1) (2019) 2551-2551.

[189] K. Kmita, C. Wirth, J. Warnau, S. Guerrero-Castillo, C. Hunte, G. Hummer, V.R.I. Kaila, K. Zwicker, U. Brandt, V. Zickermann, Accessory NUMM (NDUFS6) subunit harbors a Zn-binding site and is essential for biogenesis of mitochondrial complex I, Proceedings of the National Academy of Sciences 112(18) (2015) 5685.

[190] T. Yano, W.R. Dunham, T. Ohnishi, Characterization of the delta muH+-sensitive ubisemiquinone species (SQ(Nf)) and the interaction with cluster N2: new insight into the energy-coupled electron transfer in complex I, Biochemistry 44(5) (2005) 1744-54.

[191] V.D. Sled, T. Friedrich, H. Leif, H. Weiss, S.W. Meinhardt, Y. Fukumori, M.W. Calhoun, R.B.

Gennis, T. Ohnishi, Bacterial NADH-quinone oxidoreductases: iron-sulfur clusters and related problems, J Bioenerg Biomembr 25(4) (1993) 347-56.

[192] N. Le Breton, J.J. Wright, A.J.Y. Jones, E. Salvadori, H.R. Bridges, J. Hirst, M.M. Roessler, Using Hyperfine Electron Paramagnetic Resonance Spectroscopy to Define the Proton-Coupled Electron Transfer Reaction at Fe-S Cluster N2 in Respiratory Complex I, J Am Chem Soc 139(45) (2017) 1631916326.

[193] K. Zwicker, A. Galkin, S. Dröse, L. Grgic, S. Kerscher, U. Brandt, The Redox-Bohr Group Associated with Iron-Sulfur Cluster N2 of Complex I*, Journal of Biological Chemistry 281(32) (2006) 23013-23017.

[194] M.A. Hameedi, D.N. Grba, K.H. Richardson, A.J.Y. Jones, W. Song, M.M. Roessler, J.J. Wright, J. Hirst, A conserved arginine residue is critical for stabilizing the N2 FeS cluster in mitochondrial complex I, Journal of Biological Chemistry 296 (2021) 100474.

[195] A.J. Kowaltowski, N.C. de Souza-Pinto, R.F. Castilho, A.E. Vercesi, Mitochondria and reactive oxygen species, Free Radic Biol Med 47(4) (2009) 333-43.

[196] M.D. Brand, The sites and topology of mitochondrial superoxide production, Exp Gerontol 45(7-8) (2010) 466-72.

[197] F. Scialò, D.J. Fernández-Ayala, A. Sanz, Role of Mitochondrial Reverse Electron Transport in ROS Signaling: Potential Roles in Health and Disease, Frontiers in Physiology 8(428) (2017).

[198] Michael P. Murphy, How mitochondria produce reactive oxygen species, Biochemical Journal 417(1) (2008) 1-13.

[199] E.D. Michelakis, V. Hampl, A. Nsair, X. Wu, G. Harry, A. Haromy, R. Gurtu, S.L. Archer, Diversity in Mitochondrial Function Explains Differences in Vascular Oxygen Sensing, Circulation Research 90(12) (2002) 1307-1315.

[200] E.T. Chouchani, V.R. Pell, E. Gaude, D. Aksentijević, S.Y. Sundier, E.L. Robb, A. Logan, S.M. Nadtochiy, E.N.J. Ord, A.C. Smith, F. Eyassu, R. Shirley, C.-H. Hu, A.J. Dare, A.M. James, S. Rogatti, R.C. Hartley, S. Eaton, A.S.H. Costa, P.S. Brookes, S.M. Davidson, M.R. Duchen, K. Saeb-Parsy, M.J. Shattock, A.J. Robinson, L.M. Work, C. Frezza, T. Krieg, M.P. Murphy, Ischaemic accumulation of succinate controls reperfusion injury through mitochondrial ROS, Nature 515(7527) (2014) 431-435. [201] S. Huang, A.H. Millar, Succinate dehydrogenase: the complex roles of a simple enzyme, Current Opinion in Plant Biology 16(3) (2013) 344-349.

[202] M.R.H.A. Rasheed, G. Tarjan, Succinate Dehydrogenase Complex: An Updated Review, Archives of Pathology \& Laboratory Medicine 142(12) (2018) 1564-1570.

[203] F. Sun, X. Huo, Y. Zhai, A. Wang, J. Xu, D. Su, M. Bartlam, Z. Rao, Crystal Structure of Mitochondrial Respiratory Membrane Protein Complex II, Cell 121(7) (2005) 1043-1057.

[204] C.C. Page, C.C. Moser, X. Chen, P.L. Dutton, Natural engineering principles of electron tunnelling in biological oxidation-reduction, Nature 402(6757) (1999) 47-52.

[205] R.F. Anderson, S.S. Shinde, R. Hille, R.A. Rothery, J.H. Weiner, S. Rajagukguk, E. Maklashina, G. Cecchini, Electron-transfer pathways in the heme and quinone-binding domain of complex II (succinate dehydrogenase), Biochemistry 53(10) (2014) 1637-1646. 
[206] J. St-Pierre, J.A. Buckingham, S.J. Roebuck, M.D. Brand, Topology of Superoxide Production from Different Sites in the Mitochondrial Electron Transport Chain *, Journal of Biological Chemistry 277(47) (2002) 44784-44790.

[207] B.A.C. Ackrell, Cytopathies involving mitochondrial complex II, Molecular Aspects of Medicine 23(5) (2002) 369-384.

[208] A.S. Hoekstra, J.-P. Bayley, The role of complex II in disease, Biochimica et Biophysica Acta (BBA) - Bioenergetics 1827(5) (2013) 543-551.

[209] B.A. Ackrell, E.B. Kearney, T.P. Singer, Mammalian succinate dehydrogenase, Methods Enzymol 53 (1978) 466-83.

[210] A.R. Crofts, The Cytochrome bc1 Complex: Function in the Context of Structure, Annual Review of Physiology 66(1) (2004) 689-733.

[211] S. Iwata, J.W. Lee, K. Okada, J.K. Lee, M. Iwata, B. Rasmussen, T.A. Link, S. Ramaswamy, B.K. Jap, Complete Structure of the 11-Subunit Bovine Mitochondrial Cytochrome

bc\&lt;sub\&gt;1\&lt;/sub\&gt; Complex, Science 281(5373) (1998) 64.

[212] C.-A. Yu, L. Yu, D. Xia, Ubiquinol-Cytochrome c Oxidoreductase (Complex III), in: G.C.K.

Roberts (Ed.), Encyclopedia of Biophysics, Springer Berlin Heidelberg, Berlin, Heidelberg, 2013, pp. 2679-2684.

[213] D. Xia, L. Esser, W.-K. Tang, F. Zhou, Y. Zhou, L. Yu, C.-A. Yu, Structural analysis of cytochrome bc1 complexes: implications to the mechanism of function, Biochim Biophys Acta 1827(11-

12) (2013) 1278-1294.

[214] J.L. Cape, M.K. Bowman, D.M. Kramer, A semiquinone intermediate generated at the Q\&lt;sub\&gt;o\&lt;/sub\&gt; site of the cytochrome \&lt;em\&gt;bc\&lt;/em\&gt;\&lt;sub\&gt;1\&lt;/sub\&gt; complex: Importance for the Q-cycle and superoxide production, Proceedings of the National Academy of Sciences 104(19) (2007) 7887.

[215] S. Iwata, M. Saynovits, T.A. Link, H. Michel, Structure of a water soluble fragment of the 'Rieske' iron-sulfur protein of the bovine heart mitochondrial cytochrome bc1 complex determined by MAD phasing at 1.5 å resolution, Structure 4(5) (1996) 567-579.

[216] J.L. Smith, H. Zhang, J. Yan, G. Kurisu, W.A. Cramer, Cytochrome bc complexes: a common core of structure and function surrounded by diversity in the outlying provinces, Current Opinion in Structural Biology 14(4) (2004) 432-439.

[217] Z. Zhang, L. Huang, V.M. Shulmeister, Y.-I. Chi, K.K. Kim, L.-W. Hung, A.R. Crofts, E.A. Berry, S.-H. Kim, Electron transfer by domain movement in cytochrome bc1, Nature 392(6677) (1998) 677-684. [218] L. Esser, X. Gong, S. Yang, L. Yu, C.-A. Yu, D. Xia, Surface-modulated motion switch: Capture and release of iron-sulfur protein in the cytochrome \&lt;em\&gt;bc\&lt;/em\&gt;\&lt;sub\&gt;1\&lt;/sub\&gt; complex, Proceedings of the National Academy of Sciences 103(35) (2006) 13045.

[219] E.A. Berry, H. De Bari, L.S. Huang, Unanswered questions about the structure of cytochrome bc1 complexes, Biochim Biophys Acta 1827(11-12) (2013) 1258-77.

[220] C.L. Quinlan, A.A. Gerencser, J.R. Treberg, M.D. Brand, The Mechanism of Superoxide

Production by the Antimycin-inhibited Mitochondrial Q-cycle *, Journal of Biological Chemistry 286(36) (2011) 31361-31372.

[221] F.L. Muller, Y. Liu, H. Van Remmen, Complex III releases superoxide to both sides of the inner mitochondrial membrane, J Biol Chem 279(47) (2004) 49064-73.

[222] H. Beinert, P.J. Kiley, Fe-S proteins in sensing and regulatory functions, Curr Opin Chem Biol 3(2) (1999) 152-7.

[223] M.-P. Golinelli-Cohen, C. Bouton, Fe-S proteins acting as redox switch: new key actors of cellular adaptive responses, Current Chemical Biology 11(2) (2017) 70-88.

[224] C.A. Nurse, S. Salman, A.L. Scott, Hypoxia-regulated catecholamine secretion in chromaffin cells, Cell and Tissue Research 372(2) (2018) 433-441.

[225] C. Peers, P.J. Kemp, Acute oxygen sensing: diverse but convergent mechanisms in airway and arterial chemoreceptors, Respir Res 2(3) (2001) 145-9. 
[226] M. Jastroch, A.S. Divakaruni, S. Mookerjee, J.R. Treberg, M.D. Brand, Mitochondrial proton and electron leaks, Essays Biochem 47 (2010) 53-67.

[227] F.R. Palma, C. He, J.M. Danes, V. Paviani, D.R. Coelho, B.N. Gantner, M.G. Bonini, Mitochondrial Superoxide Dismutase: What the Established, the Intriguing, and the Novel Reveal About a Key Cellular Redox Switch, Antioxid Redox Signal 32(10) (2020) 701-714.

[228] N. Di Marzo, E. Chisci, R. Giovannoni, The Role of Hydrogen Peroxide in Redox-Dependent Signaling: Homeostatic and Pathological Responses in Mammalian Cells, Cells 7(10) (2018) 156.

[229] J. Lopez-Barneo, J.R. Lopez-Lopez, J. Urena, C. Gonzalez, Chemotransduction in the carotid body: $\mathrm{K}+$ current modulated by PO2 in type I chemoreceptor cells, Science 241(4865) (1988) 580-582.

[230] S.L. Archer, X.-C. Wu, B. Thébaud, A. Nsair, S. Bonnet, B. Tyrrell, M.S. McMurtry, K. Hashimoto, G. Harry, E.D. Michelakis, Preferential expression and function of voltage-gated, O2sensitive $\mathrm{K}+$ channels in resistance pulmonary arteries explains regional heterogeneity in hypoxic pulmonary vasoconstriction: ionic diversity in smooth muscle cells, Circulation research 95(3) (2004) 308-318.

[231] H. Kajimoto, K. Hashimoto, S.N. Bonnet, A. Haromy, G. Harry, R. Moudgil, T. Nakanishi, I. Rebeyka, B. Thébaud, E.D. Michelakis, Oxygen activates the Rho/Rho-kinase pathway and induces RhoB and ROCK-1 expression in human and rabbit ductus arteriosus by increasing mitochondria-derived reactive oxygen species: a newly recognized mechanism for sustaining ductal constriction, Circulation $115(13)$ (2007) 1777-1788.

[232] M. Uehata, T. Ishizaki, H. Satoh, T. Ono, T. Kawahara, T. Morishita, H. Tamakawa, K. Yamagami, J. Inui, M. Maekawa, Calcium sensitization of smooth muscle mediated by a Rho-associated protein kinase in hypertension, Nature 389(6654) (1997) 990-994.

[233] R.B. Howard, T. Hosokawa, M.H. Maguire, Hypoxia-induced fetoplacental vasoconstriction in perfused human placental cotyledons, American journal of obstetrics and gynecology 157(5) (1987) $1261-1266$.

[234] V. Hampl, J. Bíbová, Z. Straňák, X. Wu, E.D. Michelakis, K. Hashimoto, S.L. Archer, Hypoxic fetoplacental vasoconstriction in humans is mediated by potassium channel inhibition, American Journal of Physiology-Heart and Circulatory Physiology 283(6) (2002) H2440-H2449.

[235] G.G. Power, L.D. Longo, H.N. Wagner, D.E. Kuhl, R.E. Forster, Uneven distribution of maternal and fetal placental blood flow, as demonstrated using macroaggregates, and its response to hypoxia, The Journal of clinical investigation 46(12) (1967) 2053-2063.

[236] D.-C. Lee, R. Romero, J.-S. Kim, A.L. Tarca, D. Montenegro, B.L. Pineles, E. Kim, J. Lee, S.Y. Kim, S. Draghici, miR-210 targets iron-sulfur cluster scaffold homologue in human trophoblast cell lines: siderosis of interstitial trophoblasts as a novel pathology of preterm preeclampsia and small-forgestational-age pregnancies, The American journal of pathology 179(2) (2011) 590-602.

[237] T. Khong, F. De Wolf, W. Robertson, I. Brosens, Inadequate maternal vascular response to placentation in pregnancies complicated by pre-eclampsia and by small-for-gestational age infants, BJOG: An International Journal of Obstetrics \& Gynaecology 93(10) (1986) 1049-1059.

[238] S.T. Bauer, K.L. Cleary, Cardiopulmonary complications of pre-eclampsia, Seminars in perinatology, Elsevier, 2009, pp. 158-165.

[239] B. Sibai, G. Dekker, M. Kupferminc, Pre-eclampsia, The Lancet 365(9461) (2005) 785-799.

[240] L.E. Keyes, F.J. Armaza, S. Niermeyer, E. Vargas, D.A. Young, L.G. Moore, Intrauterine growth restriction, preeclampsia, and intrauterine mortality at high altitude in Bolivia, Pediatric research 54(1) (2003) 20-25.

[241] S.K. Palmer, L.G. Moore, D.A. Young, B. Cregger, J.C. Berman, S. Zamudio, Altered blood pressure course during normal pregnancy and increased preeclampsia at high altitude (3100 meters) in Colorado, American journal of obstetrics and gynecology 180(5) (1999) 1161-1168.

[242] F. Colleoni, N. Padmanabhan, H.-w. Yung, E.D. Watson, I. Cetin, M.C. Tissot van Patot, G.J. Burton, A.J. Murray, Suppression of mitochondrial electron transport chain function in the hypoxic human placenta: a role for miRNA-210 and protein synthesis inhibition, PloS one 8(1) (2013) e55194. 
[243] J. López-Barneo, P. González-Rodríguez, L. Gao, M.C. Fernández-Agüera, R. Pardal, P. OrtegaSáenz, Oxygen sensing by the carotid body: mechanisms and role in adaptation to hypoxia, Am J Physiol Cell Physiol 310(8) (2016) C629-42.

[244] C. Peers, Hypoxic suppression of K+ currents in type I carotid body cells: selective effect on the Ca2+-activated K+ current, Neuroscience letters 119(2) (1990) 253-256.

[245] I. Arias-Mayenco, P. González-Rodríguez, H. Torres-Torrelo, L. Gao, M.C. Fernández-Agüera, V. Bonilla-Henao, P. Ortega-Sáenz, J. López-Barneo, Acute O2 Sensing: Role of Coenzyme QH2/Q Ratio and Mitochondrial ROS Compartmentalization, Cell Metabolism 28(1) (2018) 145-158.e4.

[246] E.D. Michelakis, B. Thébaud, E.K. Weir, S.L. Archer, Hypoxic pulmonary vasoconstriction: redox regulation of $\mathrm{O} 2$-sensitive $\mathrm{K}+$ channels by a mitochondrial $\mathrm{O} 2$-sensor in resistance artery smooth muscle cells, J Mol Cell Cardiol 37(6) (2004) 1119-36.

[247] J. López-Barneo, R. Pardal, R.J. Montoro, T. Smani, J. García-Hirschfeld, J. Ureña, K+ and Ca2+ channel activity and cytosolic [Ca2+] in oxygen-sensing tissues, Respir Physiol 115(2) (1999) 215-27. [248] L. Gao, P. González-Rodríguez, P. Ortega-Sáenz, J. López-Barneo, Redox signaling in acute oxygen sensing, Redox Biol 12 (2017) 908-915.

[249] J. Koch, G. Hensley, L. Roy, S. Brown, C. Ramaciotti, C.R. Rosenfeld, Prevalence of spontaneous closure of the ductus arteriosus in neonates at a birth weight of 1000 grams or less, Pediatrics 117(4) (2006) 1113-1121.

[250] S.R. Seidner, Y.Q. Chen, P.R. Oprysko, F. Mauray, M.T. Mary, E. Lin, C. Koch, R.I. Clyman, Combined prostaglandin and nitric oxide inhibition produces anatomic remodeling and closure of the ductus arteriosus in the premature newborn baboon, Pediatric research 50(3) (2001) 365-373.

[251] H. Kajino, Y.-Q. Chen, S.R. Seidner, N. Waleh, F. Mauray, C. Roman, S. Chemtob, C.J. Koch, R.I. Clyman, Factors that increase the contractile tone of the ductus arteriosus also regulate its anatomic remodeling, American Journal of Physiology-Regulatory, Integrative and Comparative Physiology 281(1) (2001) R291-R301.

[252] E. Hermes-DeSantis, R. Clyman, Patent ductus arteriosus: pathophysiology and management, Journal of perinatology 26(1) (2006) S14-S18.

[253] Z. Hong, S. Kutty, P.T. Toth, G. Marsboom, J.M. Hammel, C. Chamberlain, J.J. Ryan, H.J. Zhang, W.W. Sharp, E. Morrow, Role of dynamin-related protein 1 (Drp1)-mediated mitochondrial fission in oxygen sensing and constriction of the ductus arteriosus, Circulation research 112(5) (2013) 802-815. [254] M. Tristani-Firouzi, H.L. Reeve, S. Tolarova, E.K. Weir, S.L. Archer, Oxygen-induced constriction of rabbit ductus arteriosus occurs via inhibition of a 4-aminopyridine-, voltage-sensitive potassium channel, The Journal of clinical investigation 98(9) (1996) 1959-1965.

[255] B. Thebaud, X.-C. Wu, H. Kajimoto, S. Bonnet, K. Hashimoto, E.D. Michelakis, S.L. Archer, Developmental absence of the O 2 sensitivity of L-type calcium channels in preterm ductus arteriosus smooth muscle cells impairs $\mathrm{O} 2$ constriction contributing to patent ductus arteriosus, Pediatric research 63(2) (2008) 176-181.

[256] R.E. Bentley, C.C. Hindmarch, K.J. Dunham-Snary, B. Snetsinger, J.D. Mewburn, A. Thébaud, P.D. Lima, B. Thébaud, S.L. Archer, The molecular mechanisms of oxygen-sensing in human ductus arteriosus smooth muscle cells: A comprehensive transcriptome profile reveals a central role for mitochondria, Genomics (2021).

[257] L. Vernis, N. El Banna, D. Baille, E. Hatem, A. Heneman, M.E. Huang, Fe-S Clusters Emerging as Targets of Therapeutic Drugs, Oxid Med Cell Longev 2017 (2017) 3647657.

[258] S.E. Wiley, A.N. Murphy, S.A. Ross, P. van der Geer, J.E. Dixon, MitoNEET is an iron-containing outer mitochondrial membrane protein that regulates oxidative capacity, Proc Natl Acad Sci U S A 104(13) (2007) 5318-23.

[259] S.E. Wiley, M.L. Paddock, E.C. Abresch, L. Gross, P. van der Geer, R. Nechushtai, A.N. Murphy, P.A. Jennings, J.E. Dixon, The outer mitochondrial membrane protein mitoNEET contains a novel redoxactive 2Fe-2S cluster, J Biol Chem 282(33) (2007) 23745-9.

[260] J. Lin, T. Zhou, K. Ye, J. Wang, Crystal structure of human mitoNEET reveals distinct groups of iron sulfur proteins, Proc Natl Acad Sci U S A 104(37) (2007) 14640-5. 
[261] J. Schlesier, M. Rohde, S. Gerhardt, O. Einsle, A Conformational Switch Triggers Nitrogenase Protection from Oxygen Damage by Shethna Protein II (FeSII), J Am Chem Soc 138(1) (2016) 239-47.

[262] G. Chen, Z. Chen, Y. Hu, P. Huang, Inhibition of mitochondrial respiration and rapid depletion of mitochondrial glutathione by beta-phenethyl isothiocyanate: mechanisms for anti-leukemia activity, Antioxid Redox Signal 15(12) (2011) 2911-21.

[263] F. Bai, F. Morcos, Y.S. Sohn, M. Darash-Yahana, C.O. Rezende, C.H. Lipper, M.L. Paddock, L. Song, Y. Luo, S.H. Holt, S. Tamir, E.A. Theodorakis, P.A. Jennings, J.N. Onuchic, R. Mittler, R. Nechushtai, The Fe-S cluster-containing NEET proteins mitoNEET and NAF-1 as chemotherapeutic targets in breast cancer, Proc Natl Acad Sci U S A 112(12) (2015) 3698-703.

[264] A.F. Salem, D. Whitaker-Menezes, A. Howell, F. Sotgia, M.P. Lisanti, Mitochondrial biogenesis in epithelial cancer cells promotes breast cancer tumor growth and confers autophagy resistance, Cell Cycle 11(22) (2012) 4174-80.

[265] Y.S. Sohn, S. Tamir, L. Song, D. Michaeli, I. Matouk, A.R. Conlan, Y. Harir, S.H. Holt, V. Shulaev, M.L. Paddock, A. Hochberg, I.Z. Cabanchick, J.N. Onuchic, P.A. Jennings, R. Nechushtai, R. Mittler, NAF-1 and mitoNEET are central to human breast cancer proliferation by maintaining mitochondrial homeostasis and promoting tumor growth, Proc Natl Acad Sci U S A 110(36) (2013) 14676-81.

[266] M. Darash-Yahana, Y. Pozniak, M. Lu, Y.S. Sohn, O. Karmi, S. Tamir, F. Bai, L. Song, P.A. Jennings, E. Pikarsky, T. Geiger, J.N. Onuchic, R. Mittler, R. Nechushtai, Breast cancer tumorigenicity is dependent on high expression levels of NAF-1 and the lability of its Fe-S clusters, Proc Natl Acad Sci U S A 113(39) (2016) 10890-5.

[267] M.L. Paddock, S.E. Wiley, H.L. Axelrod, A.E. Cohen, M. Roy, E.C. Abresch, D. Capraro, A.N. Murphy, R. Nechushtai, J.E. Dixon, P.A. Jennings, MitoNEET is a uniquely folded 2Fe 2S outer mitochondrial membrane protein stabilized by pioglitazone, Proc Natl Acad Sci U S A 104(36) (2007) 14342-7.

[268] T. Zhou, J. Lin, Y. Feng, J. Wang, Binding of reduced nicotinamide adenine dinucleotide phosphate destabilizes the iron-sulfur clusters of human mitoNEET, Biochemistry 49(44) (2010) 9604-12.

[269] J.R. Colca, W.G. McDonald, D.J. Waldon, J.W. Leone, J.M. Lull, C.A. Bannow, E.T. Lund, W.R. Mathews, Identification of a novel mitochondrial protein ("mitoNEET") cross-linked specifically by a thiazolidinedione photoprobe, Am J Physiol Endocrinol Metab 286(2) (2004) E252-60.

[270] A. Laleve, C. Vallieres, M.P. Golinelli-Cohen, C. Bouton, Z. Song, G. Pawlik, S.M. Tindall, S.V. Avery, J. Clain, B. Meunier, The antimalarial drug primaquine targets Fe-S cluster proteins and yeast respiratory growth, Redox Biol 7 (2016) 21-29.

[271] M.A. Kohanski, D.J. Dwyer, B. Hayete, C.A. Lawrence, J.J. Collins, A common mechanism of cellular death induced by bactericidal antibiotics, Cell 130(5) (2007) 797-810.

[272] D.J. Dwyer, M.A. Kohanski, B. Hayete, J.J. Collins, Gyrase inhibitors induce an oxidative damage cellular death pathway in Escherichia coli, Mol Syst Biol 3 (2007) 91.

[273] X. Duan, X. Huang, X. Wang, S. Yan, S. Guo, A.E. Abdalla, C. Huang, J. Xie, 1-Serine potentiates fluoroquinolone activity against Escherichia coli by enhancing endogenous reactive oxygen species production, J Antimicrob Chemother 71(8) (2016) 2192-9.

[274] J.E. Choby, L.A. Mike, A.A. Mashruwala, B.F. Dutter, P.M. Dunman, G.A. Sulikowski, J.M. Boyd, E.P. Skaar, A Small-Molecule Inhibitor of Iron-Sulfur Cluster Assembly Uncovers a Link between Virulence Regulation and Metabolism in Staphylococcus aureus, Cell Chem Biol 23(11) (2016) 13511361.

[275] S.A. Ralph, G.G. Van Dooren, R.F. Waller, M.J. Crawford, M.J. Fraunholz, B.J. Foth, C.J. Tonkin, D.S. Roos, G.I. McFadden, Metabolic maps and functions of the Plasmodium falciparum apicoplast, Nature Reviews Microbiology 2(3) (2004) 203-216.

[276] J.E. Gisselberg, T.A. Dellibovi-Ragheb, K.A. Matthews, G. Bosch, S.T. Prigge, The suf iron-sulfur cluster synthesis pathway is required for apicoplast maintenance in malaria parasites, PLoS Pathog 9(9) (2013) e1003655. 
[277] R.M. Cicchillo, K.-H. Lee, C. Baleanu-Gogonea, N.M. Nesbitt, C. Krebs, S.J. Booker, Escherichia coli lipoyl synthase binds two distinct [4Fe-4S] clusters per polypeptide, Biochemistry 43(37) (2004) $11770-11781$.

[278] M.W. Mather, K.W. Henry, A.B. Vaidya, Mitochondrial drug targets in apicomplexan parasites, Curr Drug Targets 8(1) (2007) 49-60.

[279] J. Berthiaume, K.B. Wallace, Adriamycin-induced oxidative mitochondrial cardiotoxicity, Cell biology and toxicology 23(1) (2007) 15-25.

[280] Y. Ichikawa, M. Ghanefar, M. Bayeva, R. Wu, A. Khechaduri, S.V.N. Prasad, R.K. Mutharasan, T.J. Naik, H. Ardehali, Cardiotoxicity of doxorubicin is mediated through mitochondrial iron accumulation, The Journal of clinical investigation 124(2) (2014) 617-630.

[281] C. Myers, The role of iron in doxorubicin-induced cardiomyopathy, Seminars in oncology, 1998, pp. 10-14.

[282] P. Pacher, L. Liaudet, P. Bai, L. Virag, J. Mabley, G. Hasko, C. Szabo, Activation of poly (ADPribose) polymerase contributes to development of doxorubicin-induced heart failure, Journal of Pharmacology and Experimental Therapeutics 300(3) (2002) 862-867.

[283] P.E. Schroeder, B.B. Hasinoff, Metabolism of the one-ring open metabolites of the cardioprotective drug dexrazoxane to its active metal-chelating form in the rat, Drug metabolism and disposition 33(9) (2005) 1367-1372.

[284] D. Lebrecht, A. Geist, U.P. Ketelsen, J. Haberstroh, B. Setzer, U. Walker, Dexrazoxane prevents doxorubicin-induced long-term cardiotoxicity and protects myocardial mitochondria from genetic and functional lesions in rats, British journal of pharmacology 151(6) (2007) 771-778.

[285] J.S. Dickey, Y. Gonzalez, B. Aryal, S. Mog, A.J. Nakamura, C.E. Redon, U. Baxa, E. Rosen, G. Cheng, J. Zielonka, Mito-tempol and dexrazoxane exhibit cardioprotective and chemotherapeutic effects through specific protein oxidation and autophagy in a syngeneic breast tumor preclinical model, PloS one 8(8) (2013) e70575.

[286] A. Batova, D. Altomare, O. Chantarasriwong, K.L. Ohlsen, K.E. Creek, Y.C. Lin, A. Messersmith, A.L. Yu, J. Yu, E.A. Theodorakis, The synthetic caged garcinia xanthone cluvenone induces cell stress and apoptosis and has immune modulatory activity, Mol Cancer Ther 9(11) (2010) 2869-78.

[287] W.J. Geldenhuys, M.O. Funk, K.F. Barnes, R.T. Carroll, Structure-based design of a thiazolidinedione which targets the mitochondrial protein mitoNEET, Bioorg Med Chem Lett 20(3) (2010) 819-23.

[288] S.J. Logan, L. Yin, W.J. Geldenhuys, M.K. Enrick, K.M. Stevanov, R.T. Carroll, V.A. Ohanyan, C.L. Kolz, W.M. Chilian, Novel thiazolidinedione mitoNEET ligand-1 acutely improves cardiac stem cell survival under oxidative stress, Basic Res Cardiol 110(2) (2015) 19.

[289] J.R. Colca, Insulin sensitizers may prevent metabolic inflammation, Biochem Pharmacol 72(2)

(2006) 125-31.

[290] H. Yki-Jarvinen, Thiazolidinediones, N Engl J Med 351(11) (2004) 1106-18.

[291] G. Kispal, K. Sipos, H. Lange, Z. Fekete, T. Bedekovics, T. Janaky, J. Bassler, D.J. Aguilar Netz, J. Balk, C. Rotte, R. Lill, Biogenesis of cytosolic ribosomes requires the essential iron-sulphur protein Rli1p and mitochondria, EMBO J 24(3) (2005) 589-98.

[292] S. Khoshnevis, T. Gross, C. Rotte, C. Baierlein, R. Ficner, H. Krebber, The iron-sulphur protein RNase L inhibitor functions in translation termination, EMBO Rep 11(3) (2010) 214-9.

[293] J. Dong, R. Lai, K. Nielsen, C.A. Fekete, H. Qiu, A.G. Hinnebusch, The essential ATP-binding cassette protein RLI1 functions in translation by promoting preinitiation complex assembly, J Biol Chem 279(40) (2004) 42157-68.

[294] K. Hirabayashi, E. Yuda, N. Tanaka, S. Katayama, K. Iwasaki, T. Matsumoto, G. Kurisu, F.W. Outten, K. Fukuyama, Y. Takahashi, Functional dynamics revealed by the structure of the SufBCD complex, a novel ATP-binding cassette $(\mathrm{ABC})$ protein that serves as a scaffold for iron-sulfur cluster biogenesis, Journal of Biological Chemistry 290(50) (2015) 29717-29731. 
[295] L. Loiseau, S. Ollagnier-de-Choudens, L. Nachin, M. Fontecave, F. Barras, Biogenesis of Fe-S cluster by the bacterial Suf system: SufS and SufE form a new type of cysteine desulfurase, Journal of Biological Chemistry 278(40) (2003) 38352-38359.

[296] M. Fontecave, S.O. De Choudens, B. Py, F. Barras, Mechanisms of iron-sulfur cluster assembly: the SUF machinery, JBIC Journal of Biological Inorganic Chemistry 10(7) (2005) 713-721.

[297] S. Wollers, G. Layer, R. Garcia-Serres, L. Signor, M. Clemancey, J.-M. Latour, M. Fontecave, S.O. de Choudens, Iron-sulfur (Fe-S) cluster assembly: the SufBCD complex is a new type of Fe-S scaffold with a flavin redox cofactor, Journal of Biological Chemistry 285(30) (2010) 23331-23341.

[298] N.M. Fast, J.C. Kissinger, D.S. Roos, P.J. Keeling, Nuclear-encoded, plastid-targeted genes suggest a single common origin for apicomplexan and dinoflagellate plastids, Molecular biology and evolution $18(3)(2001)$ 418-426.

[299] M. Lee, T. Gräwert, F. Quitterer, F. Rohdich, J. Eppinger, W. Eisenreich, A. Bacher, M. Groll, Biosynthesis of isoprenoids: crystal structure of the $[4 \mathrm{Fe}-4 \mathrm{~S}]$ cluster protein IspG, Journal of molecular biology 404(4) (2010) 600-610.

[300] I. Rekittke, J. Wiesner, R. Röhrich, U. Demmer, E. Warkentin, W. Xu, K. Troschke, M. Hintz, J.H. No, E.C. Duin, Structure of (E)-4-hydroxy-3-methyl-but-2-enyl diphosphate reductase, the terminal enzyme of the non-mevalonate pathway, Journal of the American Chemical Society 130(51) (2008) 17206-17207.

[301] F. Zepeck, T. Gräwert, J. Kaiser, N. Schramek, W. Eisenreich, A. Bacher, F. Rohdich, Biosynthesis of isoprenoids. Purification and properties of IspG protein from Escherichia coli, The Journal of organic chemistry 70(23) (2005) 9168-9174.

[302] F. Pierrel, G.R. Björk, M. Fontecave, M. Atta, Enzymatic modification of tRNAs: MiaB is an ironsulfur protein, Journal of Biological Chemistry 277(16) (2002) 13367-13370.

[303] F. Arcamone, G. Cassinelli, G. Fantini, A. Grein, P. Orezzi, C. Pol, C. Spalla, Adriamycin, 14hydroxydaimomycin, a new antitumor antibiotic from S. Peucetius var. caesius, Biotechnology and bioengineering 11(6) (1969) 1101-1110.

[304] E.A. Lefrak, J. Pitha, S. Rosenheim, J.A. Gottlieb, A clinicopathologic analysis of adriamycin cardiotoxicity, Cancer 32(2) (1973) 302-314.

[305] L.J. Steinherz, P.G. Steinherz, C.T. Tan, G. Heller, M.L. Murphy, Cardiac toxicity 4 to 20 years after completing anthracycline therapy, Jama 266(12) (1991) 1672-1677.

[306] P. Mukhopadhyay, M. Rajesh, K. Yoshihiro, G. Haskó, P. Pacher, Simple quantitative detection of mitochondrial superoxide production in live cells, Biochemical and biophysical research communications 358(1) (2007) 203-208.

[307] P. Pacher, L. Liaudet, J.G. Mabley, A. Cziráki, G. Haskó, C. Szabó, Beneficial effects of a novel ultrapotent poly (ADP-ribose) polymerase inhibitor in murine models of heart failure, International journal of molecular medicine 17(2) (2006) 369-375.

[308] X. Xu, H. Persson, D. Richardson, Molecular pharmacology of the interaction of anthracyclines with iron, Molecular pharmacology 68(2) (2005) 261-271. 